\title{
Probing vesicle dynamics within small synapses
}

\author{
Dissertation \\ zur Erlangung des Doktorgrades \\ der Mathematisch-Naturwissenschaftlichen Fakultäten \\ der Georg-August-Universität zu Göttingen
}

vorgelegt von

Edward A. Lemke

aus Berlin

Göttingen 2005 
D7

Referent: Prof. Dr. Jürgen Troe

Korreferent: Prof. Dr. Erwin Neher

Tag der mündlichen Prüfung: 27.04.2005 


\section{Contents}

List of Figures v v

List of Tables $\quad$ ix

Abstract xi

1 Introduction 1

1.1 Exo-endocytosis coupling at the synapse . . . . . . . . . . . 1

1.1 .1 Synaptic vesicle pools . . . . . . . . . . . . . . . . . . 3

1.1 .2 Mobility of vesicles in the synapse . . . . . . . . . . . . . 4

1.2 Aims of this thesis $\ldots \ldots \ldots \ldots \ldots \ldots$

1.3 Fluorescence microscopy $\ldots \ldots \ldots$. . . . . . . . . . . . . . . . . . . 7

$\begin{array}{lll}1.3 .1 & \text { The point spread and optical transfer function of a microscope } & 8\end{array}$

1.3 .2 Confocal laser scanning microscopy $\ldots \ldots \ldots \ldots \ldots$

1.3 .3 Two-photon laser scanning microscopy $\ldots \ldots \ldots$. . . . . . . 11

$\begin{array}{lll}2 & \text { Experimental methods and materials } & 13\end{array}$

2.1 Primary hippocampal cultures . . . . . . . . . . . . . . . . . 13

2.2 Fluorescent styryl dyes $\ldots \ldots \ldots \ldots$

$2.3 \quad$ Real-time imaging of small single synaptic vesicles . . . . . . . . . 16

$2.3 .1 \quad$ Simultaneous imaging of single vesicles and synapses . . . . . 18

2.3 .2 Laser scanning microscopy of single synaptic vesicles . . . . . 20

3 Computational methods and simulations 23

3.1 Monte-Carlo simulation of confocal spot detection data . . . . . . . . 23

$3.1 .1 \quad$ What does one need to know to simulate FFS experiments? . 23

3.1 .2 Monte-Carlo simulation of FFS experiments . . . . . . . . 24 
3.2 Particle tracking using non ideal photon detectors . . . . . . . . . . . 26

3.2 .1 Detectors with dead-time. . . . . . . . . . . . . . . . . . 26

3.2 .2 Distortion of the point spread function by non-ideal detection 28

$3.2 .3 \quad$ Determining the center of a PSF $\ldots \ldots$. . . . . . . . . . 29

3.2 .4 Simulation and position determination . . . . . . . . . . 30

3.2 .5 How severe is the loss of photons due to dead-time? . . . . . . 34

3.2 .6 Simulation of a 2D-Gaussian . . . . . . . . . . . . . . 36

3.3 Simulation of single synaptic vesicle tracking . . . . . . . . . . . . . . 38

$3.3 .1 \quad$ Simulation of single vesicle and synapse movement. . . . . . . 38

3.3 .2 Selection criteria for single vesicle tracking . . . . . . . . . . . 39

\begin{tabular}{lll}
\hline 4 & Results & 47
\end{tabular}

\begin{tabular}{|lll}
4.1 & Fluorescence fluctuation spectroscopy of vesicle mobility in synapses . & 47
\end{tabular}

4.1 .1 The brightness of a single vesicle in FFS experiments . . . . . 49

4.1 .2 Monte-Carlo simulations of FFS data . . . . . . . . . . . . . . 51

4.2 Optimizing a setup for single vesicle tracking . . . . . . . . . . . . . . 58

$4.2 .1 \quad$ Imaging single synaptic vesicles using two-photon microscopy. $\quad 59$

$4.3 \quad$ Single synaptic vesicle tracking using dual color imaging . . . . . . . 62

$4.3 .1 \quad$ Rapidly endocytosed vesicles . . . . . . . . . . . . . . . . . 63

4.3 .2 Slowly endocytosed vesicles . . . . . . . . . . . . . 73

4.3 .3 Spontaneous miniature events . . . . . . . . . . . . . . . 77

$4.3 .4 \quad$ Mini staining using high $\mathrm{Ca}^{2+}$-solution . . . . . . . . . . . . . 79

4.3 .5 Summary of data on single vesicle tracking . . . . . . . . . . . 84

$\begin{array}{lll}5 & \text { Discussion } & 87\end{array}$

5.1 Performance of APDs in particle tracking experiments. . . . . . . . . 88

$5.2 \quad$ A quantitative model for vesicle mobility from FFS experiments . . . 91

5.3 Single vesicle tracking . . . . . . . . . . . . . . . . . . . 93

$5.3 .1 \quad$ Mobility of single vesicles . . . . . . . . . . . . . . 96

5.3 .2 Destaining kinetics of single vesicles and synapses . . . . . . . 98

$5.3 .3 \quad$ What do rapidly and slowly endocytosed vesicles have in common? . . . . . . . . . . . . . . . . 99

5.4 Imaging vs FFS; pros and cons and the future of the techniques . . . 100

\begin{tabular}{ll}
\hline A Media and solutions & 103
\end{tabular}

A.1 Hippocampal cell culture . . . . . . . . . . . . . . . . . . . . . . 103

A.2 Measuring solutions . . . . . . . . . . . . . . . . . . . . . . 104 
\begin{tabular}{ll}
\hline B Systematic simulation of FFS data & 105
\end{tabular}

$\begin{array}{ll}\text { C Optimal setup for single vesicle tracking } & 109\end{array}$

C.1 Bleaching of FM 1-43 . . . . . . . . . . . . . . . . . . . . . . . . . . 109

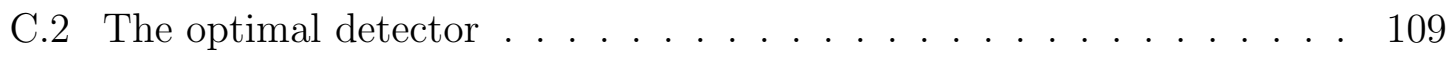

\begin{tabular}{|ll|}
\hline D Position determination with non ideal photon detectors & $\mathbf{1 1 3}$
\end{tabular}

D.1 Dead-time random number generators . . . . . . . . . . . . . . . . 113

D.2 Maximum Likelihood estimators . . . . . . . . . . . . . . . . . . . . . 114

D.2.1 ML estimator for a dead-time biased distribution . . . . . . . 115

\begin{tabular}{|lll}
\hline E Fit with four parameters for non-ideal photon counting processes & 119
\end{tabular}

\begin{tabular}{lr}
\hline Bibliography & 121
\end{tabular}

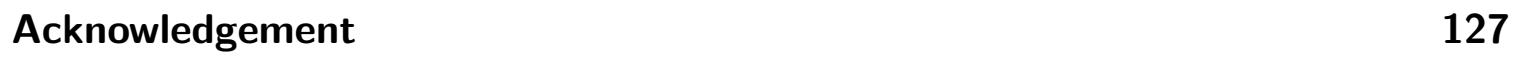

\begin{tabular}{lr}
\hline Curriculum Vitae & 129
\end{tabular} 


\section{List of Figures}

1.1 3D reconstruction of a hippocampal synapse . . . . . . . . . . . . . 2

1.2 Synaptic vesicle cycle . . . . . . . . . . . . . . . . . . 4

1.3 Optical transfer function of a lens . . . . . . . . . . . . . . . . . 9

1.4 A standard confocal fluorescent microscope . . . . . . . . . . . . . . . 10

2.1 Chemical structures of FM 1-43 and FM 5-95 . . . . . . . . . . . . . 14

$2.2 \quad$ Fluorescence and Nomarski image of a hippocampal culture . . . . . 15

2.3 Monitoring synaptic vesicle cycling with styryl dyes . . . . . . . . . . 15

2.4 Measuring chamber . . . . . . . . . . . . . . . . 16

2.5 Protocol for staining and imaging single vesicles . . . . . . . . . . . . 19

2.6 Spectral separation of FM dyes . . . . . . . . . . . . . . . . . 20

2.7 Single and multiphoton laser scanning microscope with photon counting modules . . . . . . . . . . . . . . . . . . . . . 21

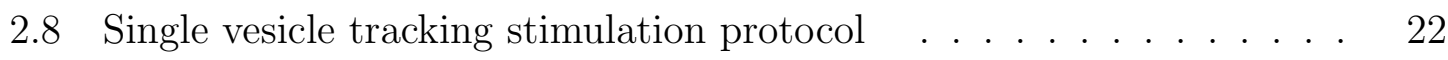

3.1 Schematic of FFS simulation . . . . . . . . . . . . . . . . . 25

3.2 Variance over mean dependence for different detector types . . . . . . 28

3.3 Bias of the PSF by non ideal counting . . . . . . . . . . . . . . . . 29

$3.4 \quad$ Precision for ideal detectors $\left(P S F_{\text {ideal }}^{\text {detected }}\right)$. . . . . . . . . . . . . . . 32

3.5 Precision for $P S F_{t_{d}}^{\text {detected }}$ of ideal and non ideal detectors . . . . . . . 33

3.6 Impact of dead-time on precision . . . . . . . . . . . . . . . . . 36

3.7 Impact of dead-time on precision in 2D . . . . . . . . . . . . . . 37

3.8 Simulation of simultaneous vesicle and synapse tracking . . . . . . . . 40

3.9 Results from simulating vesicle tracking . . . . . . . . . . . . . . . . 41

3.10 Flow chart for automated reproducible vesicle identification and tracking . . . . . . . . . . . . . . . . . . . 46

$4.1 \quad$ Variance over mean ratio in FFS $\ldots \ldots \ldots$ 
4.2 Autocorrelation analysis of fluorescent beads . . . . . . . . . . . . 50

$4.3 \quad$ Brightness of single synaptic vesicles and beads . . . . . . . . . . . 52

4.4 ACF of control and simulated data . . . . . . . . . . . . . 54

4.5 Power spectra of control and simulated data . . . . . . . . . . . . 55

4.6 Effect of high pass filtering on the variance . . . . . . . . . . . 56

4.7 Power spectra and simulation of ML-7 treatment . . . . . . . . . . 57

4.8 Power spectra and simulation of okadiac acid treatment . . . . . . . . 58

4.9 Vesicle histogram from two photon imaging . . . . . . . . . . . . . 60

4.10 RMS of single vesicles in two photon imaging . . . . . . . . . . . . 60

4.11 Rapidly endocytosed single vesicle histogram . . . . . . . . . . . . 65

4.12 Stimulus dependent release of rapidly endocytosed vesicles . . . . . . 65

4.13 Destaining kinetics of rapidly endocytosed vesicles . . . . . . . . . . . 66

4.14 Overall mobility analysis of rapidly endocytosed vesicles . . . . . . . 67

4.15 MSD analysis from rapidly endocytosed vesicles . . . . . . . . . . . . 69

4.16 Jump frequency distribution analysis from rapidly endocytosed vesicles

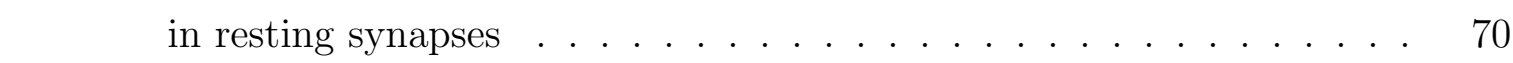

4.17 AIC for jump frequency distributions analysis of rapidly endocytosed vesicles . . . . . . . . . . . . . . . . . 71

4.18 Jump frequency distributions of stimulated rapidly endocytosed vesicles 72

4.19 K-S-test for mobility of rapidly endocytosed vesicles . . . . . . . . . . 72

4.20 Velocity of rapidly endocytosed vesicles before fusion . . . . . . . . . 73

4.21 Slowly endocytosed single vesicle histogram . . . . . . . . . . . . 74

4.22 Destaining kinetics of slowly endocytosed vesicles . . . . . . . . . . 75

4.23 Distribution of fusion time points of slowly endocytosed vesicles . . . 75

4.24 Average mobility and MSD analysis of slowly endocytosed vesicles . . 76

4.25 Jump frequency analysis of slowly endocytosed vesicles . . . . . . . . 78

4.26 Velocity of slowly endocytosed vesicles before fusion . . . . . . . . . . 79

4.27 Quantal intensity distribution from spontaneously stained cultures . 80

4.28 Destaining kinetics of the synapses and corresponding single vesicles 81

4.29 Distribution of fusion time points of spontaneously endocytosed vesicles ............................ 81

$\begin{array}{lll}4.30 & \text { Average mobility and MSD analysis of spontaneously stained vesicles } & 82\end{array}$

4.31 Velocity of rapidly endocytosed vesicles before fusion . . . . . . . . . 82

4.32 Jump frequency analysis of spontaneously endocytosed vesicles . . . . 83

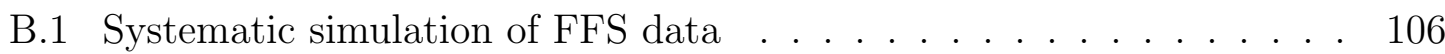


C.1 Two photon vs single photon bleaching of FM 1-43 . . . . . . . . 110

C.2 Relative detection efficiency of APD's and Hamamatsu Photon count-

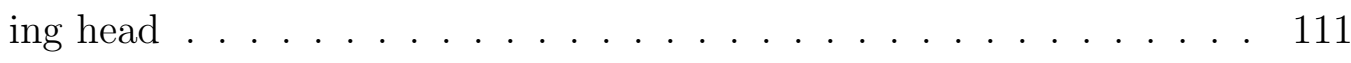

C.3 Detection characteristics of photon counting detectors . . . . . . . . . 112

D.1 Dead-time random number generators. . . . . . . . . . . . . . . . 114

D.2 Gaussian, Poisson and dead-time pdf's . . . . . . . . . . . . 117

E.1 Impact of dead-time on precision in the presence of high background

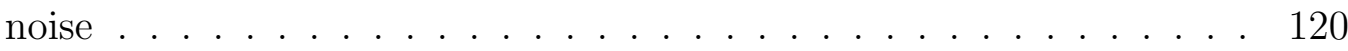




\section{List of Tables}

3.1 Summary of simulation and fit with different ML estimators . . . . . 35

3.2 Results from simulation and tracking single vesicles and synapses . . 41

\begin{tabular}{|ll|l|lll|}
\hline 4.1 & Summary of data measured by & Jordan & $(2000)$ & $\ldots$ & $\ldots$
\end{tabular}

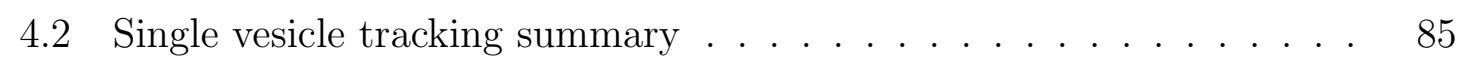

B.1 Overview of FFS simulation . . . . . . . . . . . . . . . 107

C.1 Two-photon vs single-photon bleaching of FM 1-43 . . . . . . . . . . 110 


\section{Abstract}

Sustained synaptic transmission at the hippocampal synapse $(\varnothing \sim 1 \mu \mathrm{m})$ requires continuous exocytosis and subsequent endocytosis of synaptic vesicles $(\varnothing \sim 35 \mathrm{~nm})$. The kinetics of this process have been widely studied, however, due to a lack of appropriate techniques, little is known about the mobility of small vesicles within the synapse. Fluorescence fluctuation spectroscopy (FFS) was introduced by Jordan (2000) to study vesicle dynamics in resting synapses. This study is supplemented here with Monte-Carlo simulations to propose a quantitative model of vesicle mobility. Vesicle movement was found to be slow with a diffusion coefficient of $D=5 \cdot 10^{-5} \frac{\mu m^{2}}{\mathrm{~s}}$ and restricted to a cage of $\sim 50 \mathrm{~nm}$. The effects of disabling motor proteins and treatment with a phosphatase blocker on vesicle dynamics were also analyzed using simulations.

Furthermore, the development of a single particle technique used to monitor single fluorescently labelled vesicles both, at rest and during stimulation of the synapse, is described. An automated algorithm identified single vesicles and tracked their movement in real-time. Vesicle mobility was studied in specific vesicle populations that differed in their mode of endocytosis from the plasma membrane. Results from the particle tracking and the FFS study were found to be in good agreement. Vesicle mobility was observed to increase, but was still slow during stimulation.

Additionally, a theoretical investigation of the performance of single photon counting devices with dead-time for the task of single particle tracking was performed. A Maximum Likelihood based algorithm is presented which allows to determine the position of single subresolution fluorescent particles, and yields a precision higher than expected from the total number of photons collected with these detectors. Anhaltende synaptische Transmission an der hippocampalen Synapse $(\varnothing \sim 1 \mu \mathrm{m})$ bedarf kontinuierlicher Exocytose gekoppelt and darauffolgende Endocytose von synap- 
tischen Vesikeln $(\varnothing \sim 35 \mathrm{~nm})$. Die Kinetik dieses Prozesses werden viel studiert, durch Mangel an enstprechenden Techniken weiss man allerdings nur sehr wenig über die Mobilität von diesen kleinen Vesikeln innerhalb der Synapse. Fluoreszenz Fluktuations Spectroskopie (FFS) zur Studie von Vesikel Dynamiken in unstimulierten Synapsen wurde von Jordan (2000) eingefuehrt. Diese Arbeit wurde in dieser Thesis ergänzt durch Monte-Carlo Simulationen, um ein quantitaives Model der Vesikel Beweglichkeit vorschlagen zu können. Die Vesikel Bewegung war langsam mit einem Diffusionskoeffizienten von $D=5 \cdot 10^{-5} \frac{\mu m^{2}}{s}$ und begrenzt auf einen Kaefig von $50 \mathrm{~nm}$. Die Effekte der Deaktivierung von Motor-Proteinen und nach Applikation von Phosphatase Inhibitoren auf die Vesikel Beweglichkeit wurden auch mit diesen Simulationen analysiert.

Weiterhin ist die Entwicklung einer Einzel-Partikel-Tracking Technik beschrieben, die es erlaubt einzelne fluoreszenz markierte Vesikel sowohl im unstimulierten als auch stimulierten Synapse zu untersuchen. Ein automaiserte Algorithmus identifiziert einzelene Vesikel und verfolgt Ihre Bewegung in Echtzeit. Vesikel Mobilität von verschiedenen Vesikel Populataitonen, die sich durch den Modus unterschieden mit dem sie von der Plasmamembran endozitiert wurden, wurden untersucht. Die Ergebnisse der Partikel-Tracking und der FFS Studie sind im Einklang. Vesikel Bewegung koennte leicht erhoeht werden durch Stimulation der Synapse.

Weiterhin ist eine theoretische Studie zur Leistungsfaehigkeit von Einzel-PhotonenZaehlwerken mit Totzeit fuer die Anwendung in der Einzel-Partikel-Tracking Technik beschrieben. Ein Maximum Likelihood basierter Algorithmus wird beschriben, der es erlaubte die Position von einem Object das kleiner als das Aufloesungvermoegen des Mikroskopes ist genauer zu bestimmen, als man es von der Gemsatzhal der detektierten Photonen erwarten würde. 


\section{Introduction}

In the nervous system neurons communicate with each other at specialized cell to cell contacts called synapses. The most abundant synapse is the chemical synapse, which consists of two parts: the presynapse, located at the axon of the "sending" neuron, which is filled with small vesicles containing transmitter substance, and the postsynapse, typically located at the dendrite or soma of the "receiving" neuron (see figure 1.1). Invasion of the presynapse by a specific electrical signal (termed the action potential) causes voltage-gated calcium channels in the presynaptic membrane to open. As a consequence, the $\mathrm{Ca}^{2+}$ concentration in the presynapse increases, which activates the molecular machinery regulating synaptic vesicle release (Katz and Miledi, 1967). Vesicles can then fuse with the plasma membrane in a specialized region termed the active zone. Upon fusion, the transmitter is released into the synaptic cleft, a small space between the pre- and postsynapse. Receptors in the postsynaptic membrane can detect the transmitter and trigger the opening of ion channels, thereby converting this chemical stimulus back into an electrical signal.

The process of vesicle fusion with the plasma membrane is called exocytosis. Continuous exocytosis could deplete the number of available vesicles and cause swelling of the synapse. For sustained synaptic transmission it is thus required that exocytosis is linked to a vesicle retrieval mechanism, termed endocytosis.

\subsection{Exo-endocytosis coupling at the synapse}

In the 1970's Heuser and Reese (1973) and Ceccarelli et al. (1973) performed ultrastructural studies on the synapse of the frog neuromuscular junction (NMJ), which pointed towards the existence of more than one endocytotic mechanism. Nowadays, three different classes of exo-endocytosis at synapses are reported in the literature (for review see Royle and Lagnado (2003), see also figure 1.2):

1. Clathrin mediated endocytosis (CME) was first suggested by Heuser and Reese 
Figure 1.1: 3D reconstruction of a hippocampal synapse obtained from serial sectioning and electron microscopy (modified from Schikorski and Stevens (2001)). The presynapse (blue, $\varnothing \approx 1 \mu \mathrm{m}$ ) is densely packed with small synaptic vesicles $(\varnothing \approx 35 \mathrm{~nm})$. The presynapse terminates on the postsynapse (orange), (scalebar $0.1 \mu \mathrm{m}$ ).

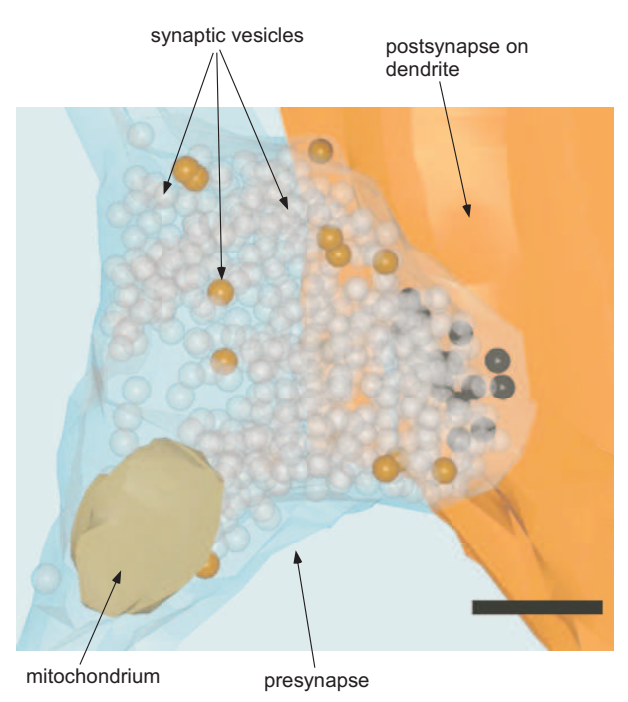

(1973). After full collapse of a vesicle into the plasma membrane, a vesicle is endocytosed from the membrane by the formation of a clathrin cage that surrounds and scavenges vesicles in its interior (for the crystal structure of clathrin refer to Fotin et al. (2004b a ) ). It is firmly established that all synapses employ clathrin mediated endocytosis (for a review see Marsh and McMahon (1999)).

2. Bulk endocytosis, where large membrane infoldings are pinched off the plasma membrane, generates large endosomes from which small vesicles can bud off (Takei et al., 1996). This mechanism can retrieve the membrane from more than one exocytotic event at once. Bulk endocytosis was shown to exists for example in the goldfish retina bipolar cell and frog NMJ (Holt et al., 2003; Richards et al., 2000). It is still a matter of debate wether or not bulk endocytosis is a common mechanism in all synapses.

3. Evidence for the existence of a kiss and run mechanism in synapses came first from the ultrastructural analysis at the frog NMJ (Ceccarelli et al., 1973). In this mode vesicles do not completely collapse during exocytosis, but rather form a fusion pore with the plasma membrane to release their content. This pore is able to close again quickly. Kiss and run was directly identified in large dense-core granule fusion within endocrine cells (a frequently used model system for synapses) by a combined approach of capacitance and amperometric measurements (Albillos et al., 1997). Further evidence for a kiss and run mode in synapses comes from imaging studies employing styryl dyes and genetically encoded fluorescent probes (for the hippocampal synapse see Aravanis et al. 
(2003b a); Gandhi and Stevens (2003)). Amperometric studies on dopamine neurons (Staal et al., 2004) and studies on transgenic flies of the Drosophila neuromuscular junction (NMJ) (Verstreken et al., 2002) also point towards the existence of a kiss and run mode.

\subsubsection{Synaptic vesicle pools}

Exo-endocytotic cycling does not occur at a constant rate during stimulation of the synapse, but shows more complicated kinetics. Combined imaging and electrophysiological studies on goldfish retinal bipolar cells for example revealed three distinct kinetic phases of release (Neves and Lagnado, 1999). The morphological correlate to these kinetic phases is called the vesicle pool. Rizzoli and Betz (2004) recently reviewed the discussion of the past decades concerning vesicle pools, and suggest that all synapses have three pools (see figure 1.2): the readily releasable pool (RRP, release $<1$ second), the recycling pool (release in $\sim$ seconds) and the reserve pool (release after $\sim$ minutes of stimulation). The hippocampal synapse, the focus of this thesis, contains approximately 200 vesicles from which $5-10 \%$ are immediately available for release and constitute the RRP (for a review see Sudhof (2000), but note, that Sudhof (2000) used a slightly different terminology for pools than Rizzoli and Betz (2004)). Under continuous stimulation of the hippocampal synapse, approximately $20 \%$ of the total vesicle population participate in recycling while the remaining vesicles are less likely to undergo exocytosis (for a review see Harata et al. (2001)).

It is still an unresolved question in neurobiology what the molecular underpinnings of these pools are, what factors determine to which pool a vesicle belongs, or whether a vesicle's "affiliation" is determined at all, or the result of a random process. There is, however, growing evidence that the future fate of a vesicle is linked to its past. It was shown that vesicles retrieved via a rapid endocytotic mechanism belong to the $\mathrm{RRP}$ and are subsequently preferentially released in hippocampal synapses Aravanis et al., 2003b; Pyle et al., 2000). Still it is unknown what constitutes such a "history tag". After Rizzoli and Betz (2004) investigated the spatial organization of vesicles belonging to the RRP using three-dimensional reconstructions of the frog NMJ, it is an open question if the "tag" is purely biochemical and/or determined by spatial criteria. 
Figure 1.2: Schematic of the synaptic vesicle cycle, highlighting the three pools (RRP, recycling pool and reserve pool) as well as the three possible modes of endocytosis (kiss and run, CME and bulk endocytosis). After endocytosis, vesicles are actively refilled with neurotransmitter (small black dots) under ATP hydrolysis. Fusion of vesicles is regulated by calcium influx. Image courtesy of Martin Wienisch. "?" depict pathways that are still a matter of debate in hippocampal synapses.

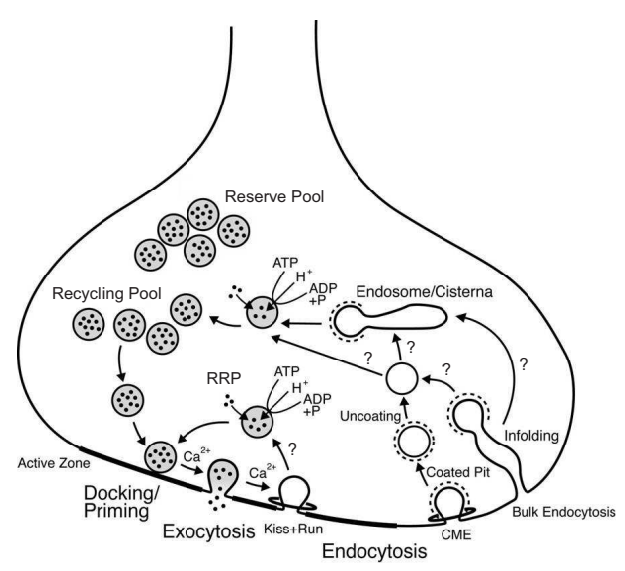

\subsubsection{Mobility of vesicles in the synapse}

The issues discussed in the previous paragraph raise additional questions: What drives a vesicle to its release site? Do vesicles arrive at the active zone randomly and are they released if they fulfill certain requirements, e.g. if they have the proper molecular identification, or are they actively transported?

Active transport of vesicles would require the action of motor proteins, which are moving along guiding tracks. In synapses, the filamentous cytoskeletal element actin is abundant and could serve as a track for the motor protein myosin, but it may also assist in maintaining the structural integrity of the synapse and/or the vesicle cluster or both (Sankaranarayanan et al., 2003; Shupliakov et al., 2002; Dunaevsky and Connor, 2000; Hirokawa et al., 1989; Evans et al., 1998). In hippocampal synapses Ryan (1999) could measure an effect on vesicle mobilization by blocking the myosin light chain kinase. This points towards an active transport mechanism in these synapses. This effect was also observed by Jordan (2000) who probed vesicle dynamics in individual presynapses using fluorescence fluctuation spectroscopy.

Application of the broadband phosphatase blocker okadaic acid caused the dispersion of the vesicle cluster in hippocampal synapses (Kraszewski et al., 1995) and frog NMJ (Betz and Henkel, 1994). These studies point towards the importance of another cytoskeletal element, called synapsin, a phosphoprotein, which is abundant in some, but not all synapses. Synapsin might serve as an element in organizing the vesicle cluster or even the vesicle pools (Hirokawa et al. (1989); De Camilli et al. (1983); 
Ryan et al. (1996a) and for a review Greengard et al. (1993)).

Some information on vesicle mobility can be deduced from studies on endocrine cells. Their comparatively big granules can be located and tracked individually, allowing to directly study granule mobility (Steyer and Almers, 1999; Steyer et al., 1997; Lang et al., 2000; Burke et al., 1997). All possible modes of granule mobility were observed, immobile (probably docked) granules, confined diffusion, free diffusion and active transport along cytoskeletal elements like actin filaments.

However, studying mammalian synaptic vesicle mobility is more difficult, since most synapses are both small and densely packed with small vesicles.

A tool for studying synaptic vesicle mobility is fluorescence recovery after photobleaching (FRAP). In FRAP, vesicles are stained with a fluorescent marker and fluorescence recovery is monitored after a spatially restricted bleaching pulse. The recovery of the fluorescence signal reflects the gradual migration of unbleached vesicles from other regions of the compartment into the bleached area. Results from these studies revealed different overall vesicle mobilities in retina bipolar cells, where vesicle mobility seems high and random (Holt et al., 2004) and in the frog NMJ or the hippocampal synapse, where fluorescence recovery was very slow (Henkel et al., 1996; Kraszewski et al., 1996). FRAP, however, has some limitations: no information can be obtained about the irreversibly bleached vesicles, vesicle subpopulations are hard to identify, the time resolution is low and the method fails in the limit of very few fluorescent particles.

Direct observation of single vesicle mobility, as possible in endocrine cells for granules, is one of the most promising approaches to probe vesicle dynamics. The only study to date reporting on direct observation of the mobility of individual small synaptic vesicles applied total internal reflection microscopy (for a review on TIRF see Axelrod et al. (1983) ) to study a preparation of goldfish retinal bipolar cells (Zenisek et al., 2000). In this study, an evanescent wave was used to selectively excite fluorescently labelled vesicles only in the vicinity of the coverslip $(\sim 100 \mathrm{~nm})$, allowing for high contrast and a very good signal-to-noise ratio. The authors were able to monitor directed vesicle movement, docking and even fusion events using off-line analysis employing single particle tracking techniques. The applicability of this technique, however, is limited to preparations where the synapse attaches to the coverslip so that vesicles 
can be illuminated by the evanescent wave. Thus this method is currently not feasible for many cell types and preparations, including the hippocampal presynapse, which of necessity terminates on a postsynapse.

Electrophysiological techniques are ideally suited to study the kinetics of synaptic transmission. This is typically done by measuring the consequence of a fusion event, as for example a capacitance increase or generation of a postsynaptic current. Thus, the technique is not very well suited for direct observation of pre-exocytotic events such as vesicle movement inside the synapse. Electron microscopy allows deep insights into the ultrastructure of the synapse but suffers from a low time resolution and provides only static pictures from which quantitative conclusions on vesicle mobility are hard to deduce.

Theoretically, real-time imaging is ideally suited to probe vesicle dynamics directly. However, as outlined in the preceding section, studies aiming at these questions are still scarce, most likely due to a lack of techniques available.

\subsection{Aims of this thesis}

The aim of this thesis was to study single vesicle dynamics in the small synapses of the hippocampal neuron in real-time. Two different approaches will be presented:

1. Jordan (2000) centered a laser focus on a hippocampal synapse and monitored fluorescence fluctuations arising from the movement of fluorescently labelled vesicles in the detection volume. The techniques allowed to propose a qualitative model of vesicle mobility in resting synapses and to directly probe the qualitative effect of myosin light chain kinase blockage and okadaic acid treatment on vesicle mobility. In this thesis, additional experiments in combination with MonteCarlo simulations were used to expand the technique from Jordan (2000) to a quantitative tool, and a model for synaptic vesicle mobility in resting and pharmacologically treated hippocampal presynapses will be presented.

2. As outlined above, direct observation of time resolved single vesicle movement is one of the most promising approaches to the study of synaptic vesicle mobility. The second study of this thesis aimed to achieve this using single particle tracking techniques. Individual vesicles were fluorescently labelled and their mobility was analyzed during resting conditions and during continuous arrival of action 
potentials at the presynapse. Furthermore, vesicles with different "histories" were investigated. This single particle tracking technique required extensive method development, theoretically as well as technically. The development of a setup specialized to fulfill the task of single synaptic vesicle tracking will be outlined. This setup employed highly sensitive avalanche photo diodes as detectors. Underlying principles in using these detectors for particle tracking experiments were analyzed theoretically, uncovering an unexpected feature of these detectors.

Both techniques for studying synaptic vesicle mobility rely on fluorescence spectroscopical and microscopical techniques, and some of the basics in fluorescence spectroscopy and microscopy are summarized in the following section.

\subsection{Fluorescence microscopy}

If a fluorescent object is moved out of focus in standard wide field fluorescence microscopes, it becomes blurred and the image appears unsharp. The average fluorescence intensity collected by an infinitely large detector, however, remains constant. In biological imaging, the fluorescent sample is typically three-dimensional, so that at any given focus plane, there is a contribution of some out-of-focus fluorescence to the image, thus lowering the contrast of in-focus objects. This effect is of course undesirable and it was early understood that in biological applications some form of optical sectioning is necessary to provide the desired contrast. This is achieved e.g. in a confocal or multi-photon excitation microscope.

The seminal ideas for the modern confocal microscope were developed in the early 1950's (Young and Roberts, 1951), only after the development of the laser, however, was a confocal laser scanning microscope engineered that could be applied to biology (Brakenhoff et al., 1979; Brakenhoff, 1979). The confocal microscope is not the only technique employing optical sectioning, but it is one of the most popular in modern biological research.

In the following sections, some fundamental principles in confocal microscopy that are useful to recall when reading this thesis will be summarized. For a detailed description on confocal microscopy and its biological applications please refer to e.g. Pawley (1989) or Corle and Kino (1996). 


\subsubsection{The point spread and optical transfer function of a microscope}

When a lens is illuminated by a perfect point source, the point's resulting image on a detector contains transverse spatial amplitude variations that are described by the amplitude point spread function, $P S F_{A m p}=h(x, y)$. The point spread function of a spherical lens (neglecting any form of aberration and assuming paraxiality) can be shown to have the form of the Airy function

$$
h(r)=\frac{2 J_{1}(v)}{v},
$$

where $J_{1}(v)$ is the first order Bessel function, $\mathrm{r}$ being the distance from the center point of the image and $v=\frac{2 \pi}{\lambda} r\left(n \cdot \sin \theta_{\max }\right) . \lambda$ is the wavelength in free space and the term in brackets is the numerical aperture of the lens $N A=n \cdot \sin \theta_{\max }, \mathrm{n}$ is the refractive index of the medium and $\theta_{\max }$ is the half-angle of the acceptance cone of the lens.

The width at half amplitude of the first lobe of the airy pattern is defined as the full width at half-maximum (FWHM), which is often used as an estimate of the resolution power of the lens

$$
F W H M_{\text {lateral }}=\frac{0.51 \lambda}{n \cdot \sin \theta_{\max }}=\frac{0.51 \lambda}{N A} .
$$

The diameter of the first central lobe of the Airy pattern (from zero point to zero point) is defined as 1 Airy unit (AU). In experiments, a detector does not measure the amplitude of the PSF, but the intensity of the PSF, which is related to the amplitude by $P S F_{\text {Int }}=\left|P S F_{A m p}\right|^{2}$ or $I_{h}(x, y)=|h(x, y)|^{2}$.

Another useful measure of optical performance arises when Fourier theory is applied to optics. From the perspective of Fourier optics, the finite size of an objective lens causes the image of a perfect point source to be smeared out because the lens size limits the spatial frequencies that the lens can transmit. Figure 1.3 shows the profile of the optical transfer function (OTF) which is related to the intensity PSF by fourier transformation.

$$
\operatorname{OTF}\left(k_{x}, k_{y}\right)=\int_{-\infty}^{+\infty} \int_{-\infty}^{+\infty}|h(x, y)|^{2} e^{-j\left(k_{x} x+k_{y} y\right)} d x d y
$$




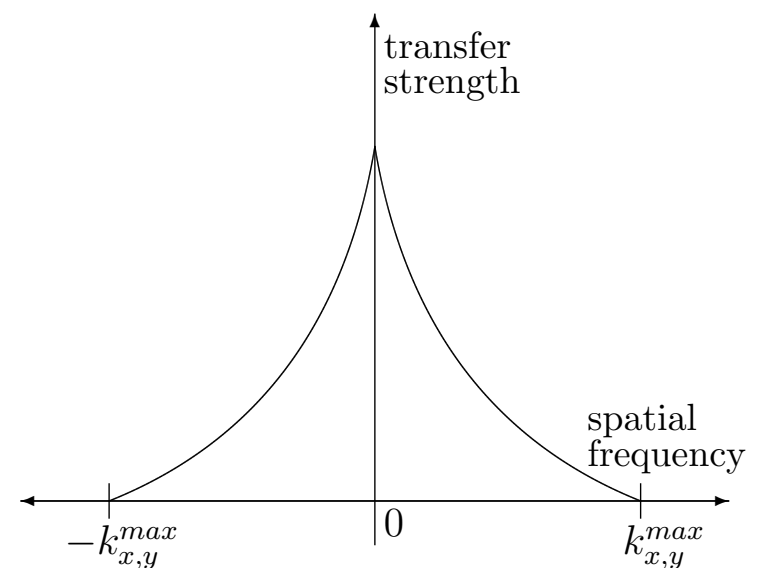

Figure 1.3: Approximate shape of the optical transfer Function (OTF) for a spherical lens. The OTF decays towards higher frequencies up until the maximum transmittable frequencies $k_{x}^{\max }$ and $k_{y}^{\max }$.

with $k_{x}$ and $k_{y}$ being the frequencies to which the optical system can respond. One can see in figure 1.3, that the OTF is smooth and declines towards higher frequencies. The highest transmittable frequencies $k_{x}^{\max }$ and $k_{y}^{\max }$ characterize the resolution of the optical system.

Other useful definitions of resolution are the Rayleigh criterion and the Sparrow limit, which have a wider applicability than the FWHM of the PSF. For the purpose of this thesis, however, the FWHM of the PSF criterion will be used, since the PSF of a microscope is conveniently accessible by either imaging of fluorescent subresolution particles, or using fluorescence correlation spectroscopy techniques (Magde et al., 1972, 1974; Thompson, 1989).

\subsubsection{Confocal laser scanning microscopy}

Figure 1.4 shows a schematic of a standard confocal fluorescence microscope. The optical sectioning effect is achieved by placing a small pinhole in front of the detector, in a plane conjugated to the focal plane. Regions of the sample that are not in focus will appear defocused, and light rays originating in these regions will be projected off-center onto the "pinhole" wall, thus being filtered out. Only in-focus light can pass through the pinhole. This type of microscope can typically be applied to spectroscopic measurements, e.g. in fluorescence correlation spectroscopy (Magde et al. 1972, 1974; Thompson, 1989). To generate an image, the sample has to be scanned, either by object scanning or by scanning the illumination point in a raster. The faster method is to scan the laser, typically by using galvanometric mirrors, or acusto-optic deflectors for very fast scanning. The signal of the detector is subsequently digitized 


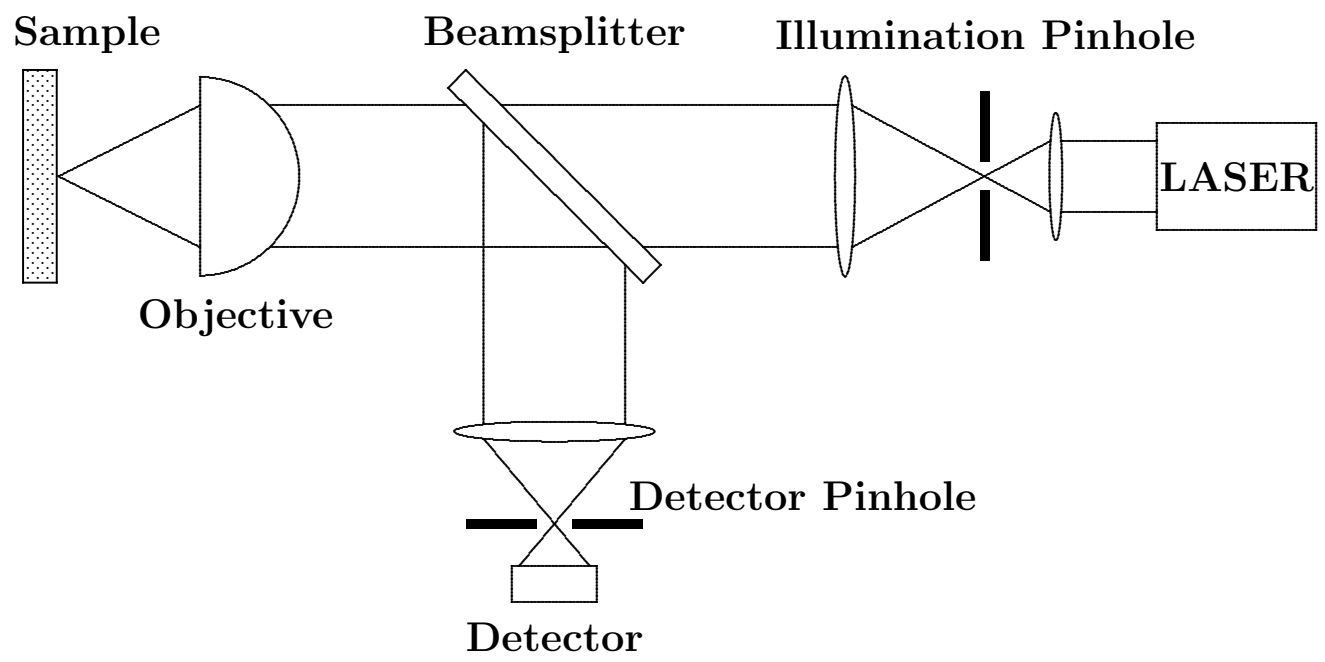

Figure 1.4: Schematic of a two-pinhole confocal microscope. The illumination pinhole is used to "clean" the beam of the LASER light source (spatial filtering of low frequency components arising from e.g. dust particles). The emission light of the fluorescent sample is then separated from the excitation light via a beamsplitter and focussed on the detector pinhole. This pinhole is positioned in a conjugated focal plane of the microscope, so that only light originating from the in-focus plane can pass through the pinhole to the detector, and rays from out-of-focus regions are rejected.

to generate an image. The correct choice of pixel size is of major importance, so that all information transferred by the optical system to the detector can be used. Choosing too large a pixel size can cause distinct objects to fall together within one pixel. On the other hand, too small (and thus too many) pixels are often undesirable, since image acquisition time increases drastically with the number of scan points in a scanning microscope. However, according to the Nyquist theorem, the spatial sampling frequency should be twice as high as the maximum transferred spatial frequency $\left(k_{\max }\right.$, see figure 1.3). Further oversampling can additionally enhance position determination (Heintzmann, 1999) .

The pinhole in a confocal microscope makes it possible to influence the optical performance of the microscope. In the limit of large pinhole sizes, the lateral and axial in-focus resolution are not much different from a standard microscope (see for $F W H M_{\text {lateral }}$ equation 1.1). However, even at large pinhole size ( $\left.>1 \mathrm{AU}\right)$ the pinhole is not obsolete, since remaining out-of-focus fluorescence is blocked by the pinhole, which to some degree prevents the deterioration of the image contrast by background fluorescence. In the limit of an infinitely small pinhole $(\sim$ pinhole $\varnothing<0.25$ AU) the 
optical resolution can be approximated using

$$
F H W M_{\text {lateral }} \approx \frac{0.37 \lambda_{\text {em }}}{N A},
$$

with $\lambda_{e m}$ being the fluorescence emission wavelength. According to this definition, the lateral resolution of a confocal microscope could be up to $27 \%$ better than in a standard microscope. It should be noted that this improvement is more a consequence of this definition (FWHM) of resolution rather than a true gain in resolution, and that it vanishes almost completely when using other definitions for resolution such as the aforementioned Rayleigh or Sparrow criteria. The axial resolution is critically dependent on the pinhole size, and can be approximated for the infinitely small pinhole to be

$$
F H W M_{a x i a l} \approx \frac{0.64 \lambda_{e m}}{\left(n-\sqrt{n^{2}-N A^{2}}\right)} .
$$

While the given equations are only approximations, the most important thing to note about resolution is that the lateral resolution scales with the first order of the NA, while axial resolution scales with the second order.

\subsubsection{Two-photon laser scanning microscopy}

Another type of microscope capable of optical sectioning uses a pulsed two-photon laser for excitation (usage of a pinhole is not anymore required (Denk et al., 1990)). This approach is based on the phenomenon that a fluorophore can be excited by the simultaneous absorption of two photons (Göppert-Mayer, 1931; Kaiser and Garrett, 1961). Two-photon excitation depends on the square of the intensity distribution, thus the two-photon excitation PSF decays fast and is basically limited to the focus volume. This rapid spatial decay is the biggest advantage of two photon microscopy, as only in-focus fluorochromes are excited. Additionally, out-of-focus regions of the sample are not bleached. In two-photon microcopy, the excitation volume and the detection volume are superimposed, and the PSF is described by the Gaussian-Lorentzian illumination profile (Berland et al., 1995):

$$
I(r, z)=\frac{2 I_{0} w_{0}^{2}}{\pi w_{z}^{2}} \exp \left(-\frac{2 r^{2}}{w_{z}^{2}}\right)
$$

with $w_{z}^{2}=w_{0}^{2}\left(1+\left(\frac{z}{z_{R}}\right)\right)$, and $z_{r}=\frac{\pi w_{0}^{2}}{\lambda}$. The focal volume can also be approximated by the 3D Gaussian illumination profile (Brown et al., 1999) 


$$
I(r, z)=I_{0} \exp \left[-\left(\frac{2 r^{2}}{w_{r}^{2}}+\frac{2 z^{2}}{w_{z}^{2}}\right)\right]
$$

and $w_{r}$ and $w_{z}$ being the $e^{-2}$ radial and axial waist radii respectively.

This allows us to calculate an approximate FWHM for the two photon (TPM) microscope with

$$
F H W M_{\text {lateral }}^{T P}=\sqrt{2 \ln 2} \cdot w_{r}=\frac{2.6 \lambda_{e x}}{2 \pi N A}
$$

and

$$
F H W M_{\text {axial }}^{T P}=\sqrt{2 \ln 2} \cdot w_{z}=\frac{8.8 n \lambda_{e x}}{2 \pi N A^{2}},
$$

with $\lambda_{e x}$ being the excitation wavelength, which is typically twice as large as the corresponding single photon wavelength.

Example The above equations for lateral and axial resolution are not exact for high numerical aperture objectives. However, they are reasonably accurate for estimating the intensity PSF. The following example shows the approximate FWHM resolutions for the case of a high numerical aperture water objective as used in this thesis (with $n_{\mathrm{H}_{2} \mathrm{O}}=1.33, \mathrm{NA}=1.2$ ).

\begin{tabular}{|l|c|c|c|}
\hline \multicolumn{2}{|l|}{} & $\begin{array}{c}F W H M_{\text {lateral }} \\
{[\mathrm{nm}]}\end{array}$ & $\begin{array}{c}F W H M_{\text {axial }} \\
{[\mathrm{nm}]}\end{array}$ \\
\hline $\begin{array}{l}\text { confocal microscope (pin- } \\
\text { hole }<0.25 \mathrm{AU})\end{array}$ & $\lambda_{e m}=530$ & 163 & 452.6 \\
\hline two photon microscope & $\lambda_{e x}=900$ & 362 & 1165 \\
\hline
\end{tabular}

In biological experiments, the pinhole size is often set to values larger than 0.25 $\mathrm{AU}$, so that the resolution of the confocal microscope and the two-photon microscope approximate each other. For choosing the optimal pinhole size to maximize the signal to noise ratio please refer to Sandison et al. (1995); Sheppard et al. (1995) Approximate equations that allow to estimate the maximum transmittable frequency $\left(k_{x, y}^{\max }\right)$ from the NA and $\lambda$ are given in Heintzmann (2005). 


\section{Experimental methods and materials}

\subsection{Primary hippocampal cultures}

Dissociated hippocampal cultures were prepared from 1-3 day old Wistar rats according to previous protocols (Pyle et al., 2000; Mozhayeva et al., 2002; Malgaroli and Tsien, 1992). In brief, postnatal rats were anesthesized and sacrificed by decapitation. The CA1-CA3 brain region was isolated with forceps and cleaned of other tissue, cut into smaller slices and washed at $4^{\circ} \mathrm{C}$ in Hanks's solution by repeated suspension and decanting (for media and solutions refer to appendix A) . The crude cell extract was digested for $10 \mathrm{~min}$ at $37^{\circ} \mathrm{C}$ in digestion solution and washed again at $4^{\circ} \mathrm{C}$. Cells were then dissociated gently by trituration with a siliconized glass pipette, pelleted by centrifugation and resuspended in plating medium. Afterwards the dissociated cells were plated onto matrigel-coated glass coverslips $(\varnothing=18 \mathrm{~mm}$ and $0.13-0.16 \mathrm{~mm}$ thickness, Assistent, Sondheim/Rhön, Germany). The cell culture was allowed to grow in medium containing $20 \%$ FCS for one day. The medium was then changed to $10 \%$ FCS and the cytostaticum ARA-C was added to control astrocyte cell proliferation. Cells were grown in a humidified incubator with $95 \%$ water and $5 \% \mathrm{CO}_{2}$, so that the astrocytes formed a homogeneous feeding layer under the hippocampal neurons. In-vitro neurons were used for experiments after 14-20 days.

\subsection{Fluorescent styryl dyes}

Betz et al. (1992) introduced FM dyes as a powerful tool to monitor synaptic activity (see figure 2.1 for chemical structures of dyes). Vesicles in synapses can be stained and subsequently destained with these dyes by controlled triggering of neuronal action potentials. The hydrophobic tail of these dyes inserts into all accessible membranes if the dye is applied to the external solution of a culture, while the hydrophilic head 

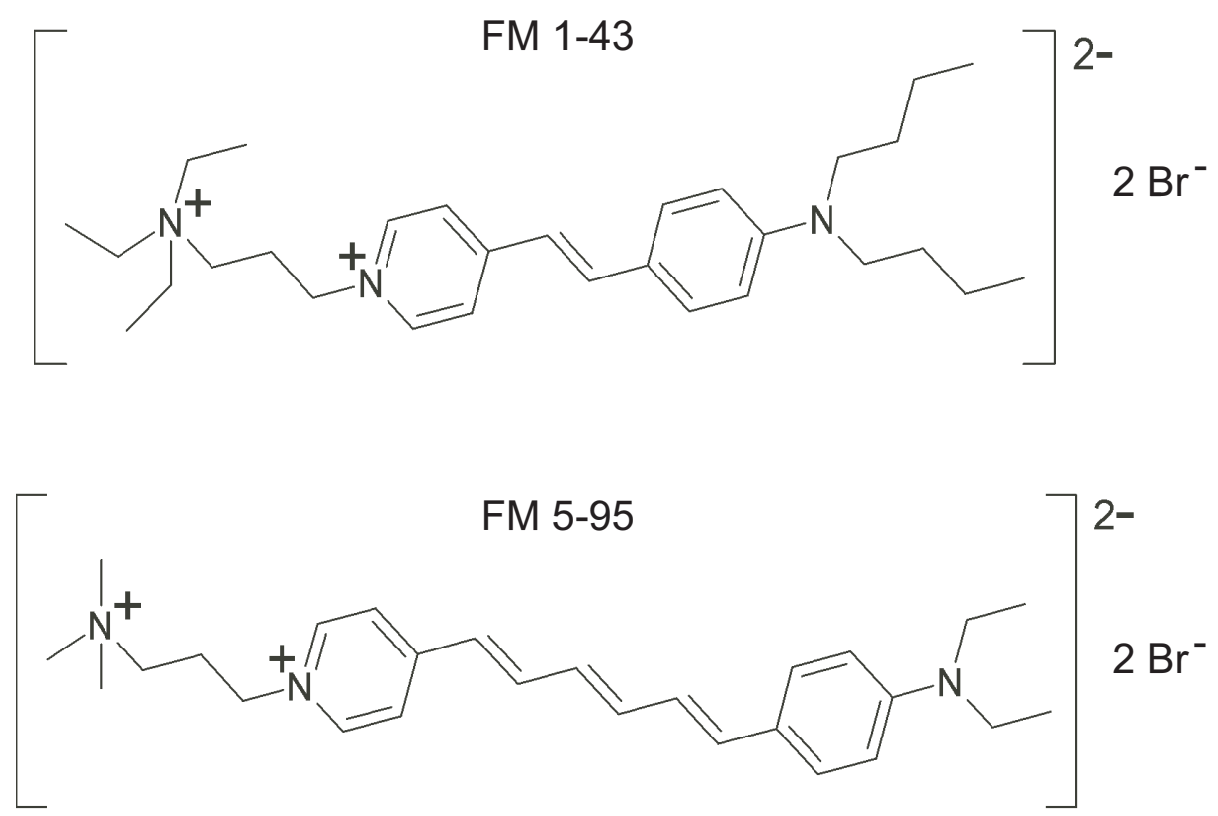

Figure 2.1: The chemical structure of the green fluorescent N-(3triethylammoniumpropyl)-4- (4-(dibutylamino)styryl)pyridinium dibromide (FM 1-43) and the red fluorescent N-(3-trimethyl-ammoniumpropyl)-4- (6-(4(diethylamino)phenyl)hexatrienyl) pyridinium dibromide (FM 5-95). Introduction of a longer conjugated $\pi$ system causes a red shift in the emission spectra (see fig. 2.6).

group prevents the dye from passing through completely. If exocytosis is triggered by arrival of action potentials, vesicles fuse with the plasma membrane and the dye also inserts into these membranes. Exocytosis in turn is coupled to vesicle retrieval mechanisms, and all vesicles that are endocytosed from the plasma membrane during presence of the dye will scavenge the dye in their interior. The dye is then washed off from the extracellular medium. If the culture is then imaged with a microscope, fluorescent spots are visible that originate from structures containing dye in their interior (see figure 2.2 for a stained hippocampal culture). If exocytosis is triggered again, stained vesicles fuse again with the plasma membrane and release the dye into the external medium, which can be monitored by an intensity drop in the fluorescence image. Figure 2.3 summarizes the FM staining and destaining protocol.

\section{The measuring configuration}

A chamber designed to hold coverslips with growing neurons was mounted on an inverted microscope (see below for details). The chamber was equipped with a global and a local perfusion system, as well as with a fluid level sensor connected to a 

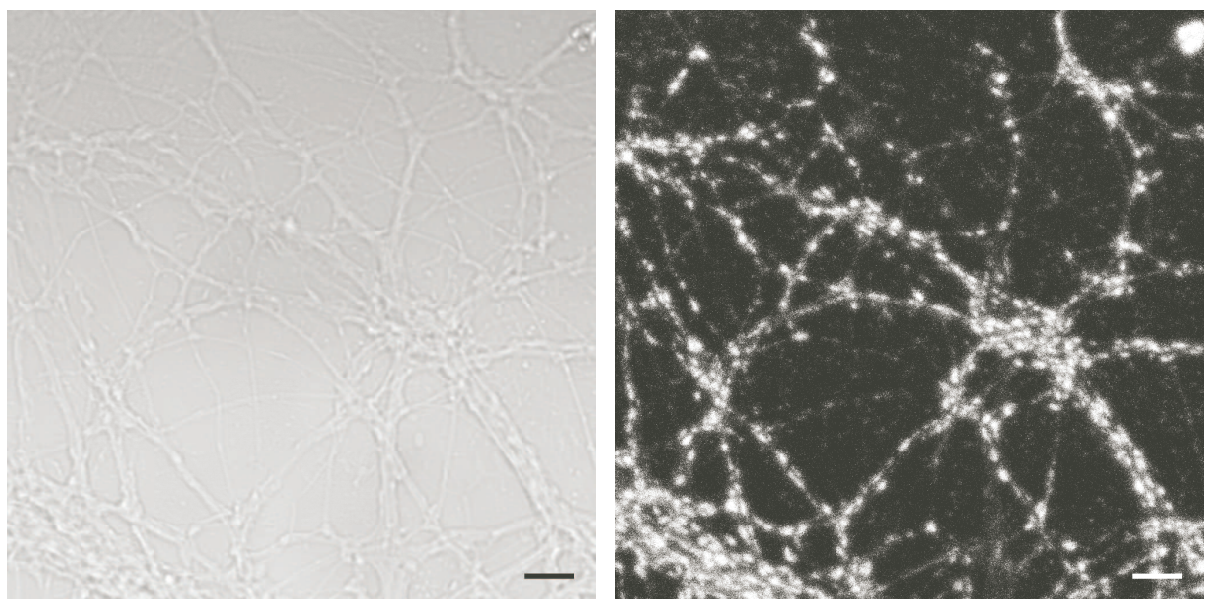

Figure 2.2: $\quad$ A hippocampal culture was stained by applying 600 APs @ $20 \mathrm{~Hz}$ in the presence of FM dye using electric field stimulation (see 2.4 for details). The left image shows a differential interference contrast image (Nomarski image), the right a fluorescence image of the same region of the culture (scale bars $=5 \mu \mathrm{m}$ ).

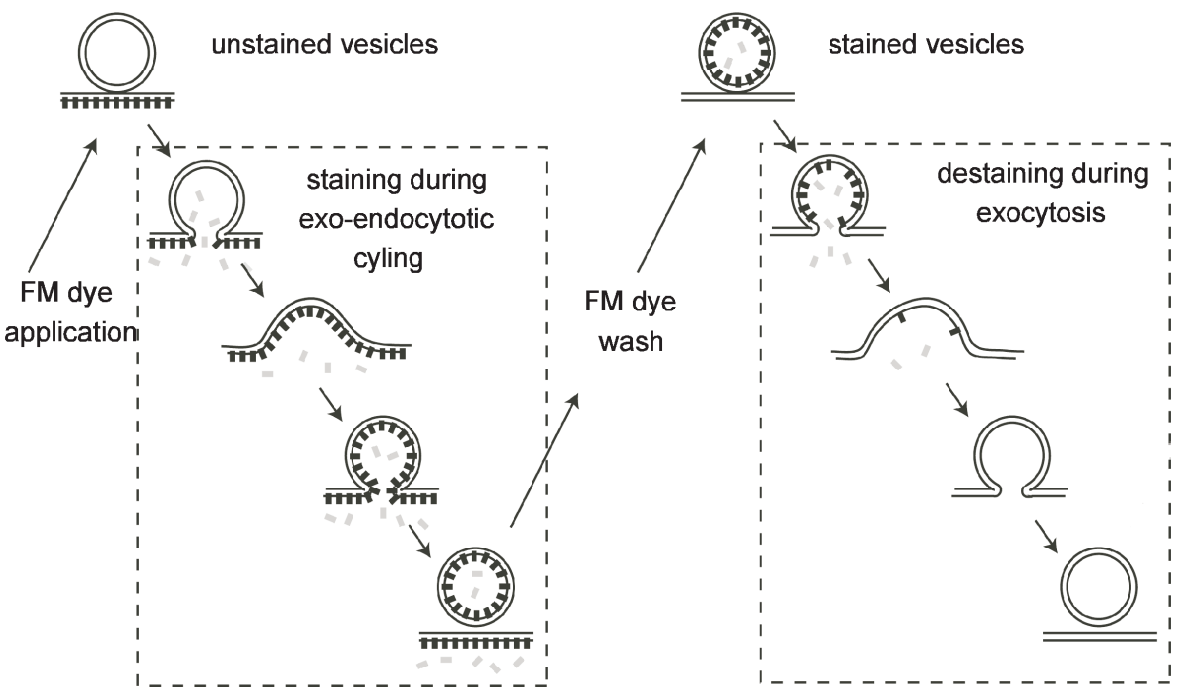

Figure 2.3: Insertion of the FM dyes (grey bars) into the plasmamembrane causes a fluorescent increase of the dye (black bars). The left part of the image shows the protocol for loading the dye. The right part shows how a successful stained vesicle can be subsequently destained. Image modified from Ryan et al. (1993), courtesy of J. Klingauf. 
Figure 2.4: The measuring chamber was equipped with two spaced platinum wires for generating field potentials, a global (not shown) and local perfusion system, a fluid level sensor and a small tube connected to a pump.

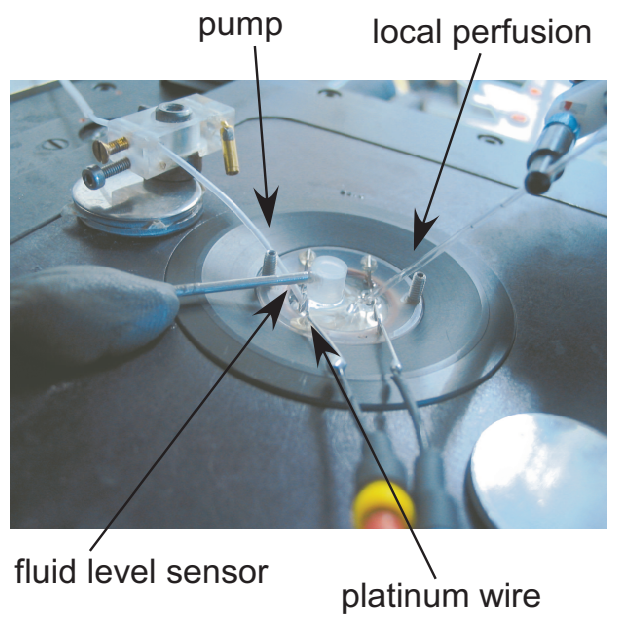

pump, which was able to regulate the fluid level with only minimal perturbance of the chamber (see figure 2.4). Action potentials were elicited in the cultured neurons by electric field stimulation. The electric field could be generated by application of short current pulses to two one-centimeter spaced platinum wires at the bottom of the culture. $1 \mathrm{~ms}$ second pulses of $40 \mathrm{~mA}$ were generated by a stimulus generator (model A 385, WPI, Berlin, Germany). The number and frequency of the pulses were controlled using a Master-8 pulse generator (A.M.P.I., Jerusalem, Israel). The chamber was made from Kel-F (teflon type material) to minimize unspecific binding of FM dye to the chamber itself.

\subsection{Real-time imaging of small single synaptic vesicles}

For staining and identification of single vesicles, a two-step one-color protocol was used (Murthy and Stevens, 1998; Aravanis et al., 2003b; Ryan et al., 1997), (see figure 2.5. If not stated differently in the text, for minimal staining $16 \mu M$ FM 1-43 was applied for a brief period of 10-15 s to the external medium, while the hippocampal culture was stimulated with 2-5 APs at $5 \mathrm{~Hz}$. After this minimal staining the culture was washed with medium containing low $\mathrm{Ca}^{2+}$ concentrations to reduce spontaneous activity in the culture (see appendix A.2 for media and solutions). Spots having an appearance similar to that of subresolution particles became visible (i.e. spots having a similar point spread function as $40 \mathrm{~nm}$ fluorescent beads; 505/515 fluorescent microspheres, Molecular Probes, Eugen, Oregon). The culture was stimulated again with 3 times 600 APs to release all vesicular fluorescence. The intensity of the fluorescent spots was measured before and after the destaining stimulus by integrating 
the fluorescence signal in a region of interest (ROI; specific sizes will be given in the text). Subtracting the residual fluorescence signal after the destaining stimulus from the one before the destaining stimulus yields the amount of releasable fluorescence $\Delta F$ (in number of photons released) in the ROI. However, the spots visible after minimal staining can, in principle, originate from single vesicles, clustered vesicles, or unspecific staining of the culture as well as non-synaptic structures which are not distinguishable from each other in images with low contrast. Thus, functional synapses at a ROI have to be identified in a high contrast image. After each experiment using the mild stimulation protocol the complete vesicle recycling pool of the synapses were stained with 600 APs @ $20 \mathrm{~Hz}$ (strong stimulation) in the presence of FM 1-43 (1 min). Functional synapses then appear as bright fluorescent spots $(\varnothing \sim 1 \mu \mathrm{m})$ that can be triggered to loose their fluorescence upon further stimulation (3 times 600 APs).

$\Delta F$ values of fluorescent spots (after minimal staining of the culture) of only ROIs where a functional synapse was identified (using the strong stimulation protocol) were plotted in histograms, which typically resulted in a distribution showing up to three peaks (see for example figure 4.3 in the results section). Since vesicle staining was shown to be quantal (similar dye uptake for all vesicles), these three peaks correspond to fluorescent puncta that represent either one, two or three fluorescently stained vesicles in the ROI (Murthy and Stevens, 1998; Aravanis et al., 2003b; Ryan et al., 1997). The width of each peak in the histogram has two origins (Murthy and Stevens, 1998): The measuring error, and the variability in the size of vesicles. The measurement error is the sum of the errors in the intensity measurement of the two images (before and after the destaining stimulus), that is $c_{m}^{2}\left((\mu \cdot k+r)^{2}+r^{2}\right)$, with $k \cdot \mu$ (in detected photons) the intensity at the center of the $k^{\text {th }}$ peak, $\mathrm{r}$ the residual fluorescence after complete destaining which was found to be roughly constant $(r=40$ photons $)$ and $c_{m}$ the coefficient of variation for the intensity measurement. This coefficient was determined by repeated measurements of green fluorescent spots and was found to be approximately constant with $c_{m}=0.8$. The coefficient of variation in vesicle size was adapted from Murthy and Stevens $(1998)$ to be $c_{v}=0.2$. The error resulting from size variations is given by $c_{v}^{2} \cdot \mu^{2} \cdot k$. The histograms (quantal histogram with three peaks) can be fitted according to a model suggested by Murthy and Stevens (1998)

$$
\sum_{k=1}^{3} A_{k} \cdot \exp \left(-\frac{1}{2} \cdot \frac{\left(x-\left(\mu \cdot k+\mu_{o f f s e t}\right)\right)^{2}}{c_{m}^{2}\left((\mu \cdot k+r)^{2}+r^{2}\right)+c_{v}^{2} \cdot \mu^{2} \cdot k}\right) .
$$


The only fit parameters are the amplitude of each peak $A_{k}$ and the peak spacing $\mu$, which corresponds to the center of the first peak $(\mathrm{k}=1)$, and thus constitutes the average fluorescence contribution of a single vesicle. The parameter $\mu_{\text {offset }}$ depicts a constant intensity offset of the parameter $\mu$ and was typically 0 . Please note that this parameter was not described in the original model of Murthy and Stevens (1998). The meaning of this parameter and why and when it can take on values different from 0 is explained in detail in section 4.3 .

In the two-step one-color protocol a time series could also be acquired (after the minimal staining, see figure 2.5p. Please note that intensities are always analyzed by selecting the optimal plane in a z-stack. However, analysis of the time series could be used to monitor the real-time properties of individual fluorescent puncta. Off-line analysis of the positions of the spots (particle tracking) allowed study of their mobility.

\subsubsection{Simultaneous imaging of single vesicles and synapses}

In the preceding section, a two-step one-color protocol was outlined which was used to determine the fluorescence contribution of single synaptic vesicles. It will be outlined in section 4.2 that for the purpose of reproducible single vesicle tracking, the two-step one-color protocol was not sufficient and had to be modified to a two-step dual-color protocol. This protocol employed staining with the green fluorescent dye FM 1-43 and with the red-shifted version FM 5-95. Figure 2.5 outlines the two-step dual-color staining protocol. The main difference to the two-step one-color protocol is that the strong staining to identify functional synapses was performed first with FM 5-95. The red fluorescent dye was then washed out and minimal staining was performed subsequently with FM 1-43. Strongly stained red fluorescent synapses and green fluorescent spots were imaged simultaneously in two separate detection channels (see next paragraph). As in the two-step one-color protocol all intensities of fluorescent objects were analyzed form z-stack recordings.

For real-time studies of vesicle mobility analysis, the time series was analyzed using particle tracking techniques (see section 3.3 for details). All analysis was performed off-line.

The particle tracking algorithm will be explained in detail in section 3.3 . 

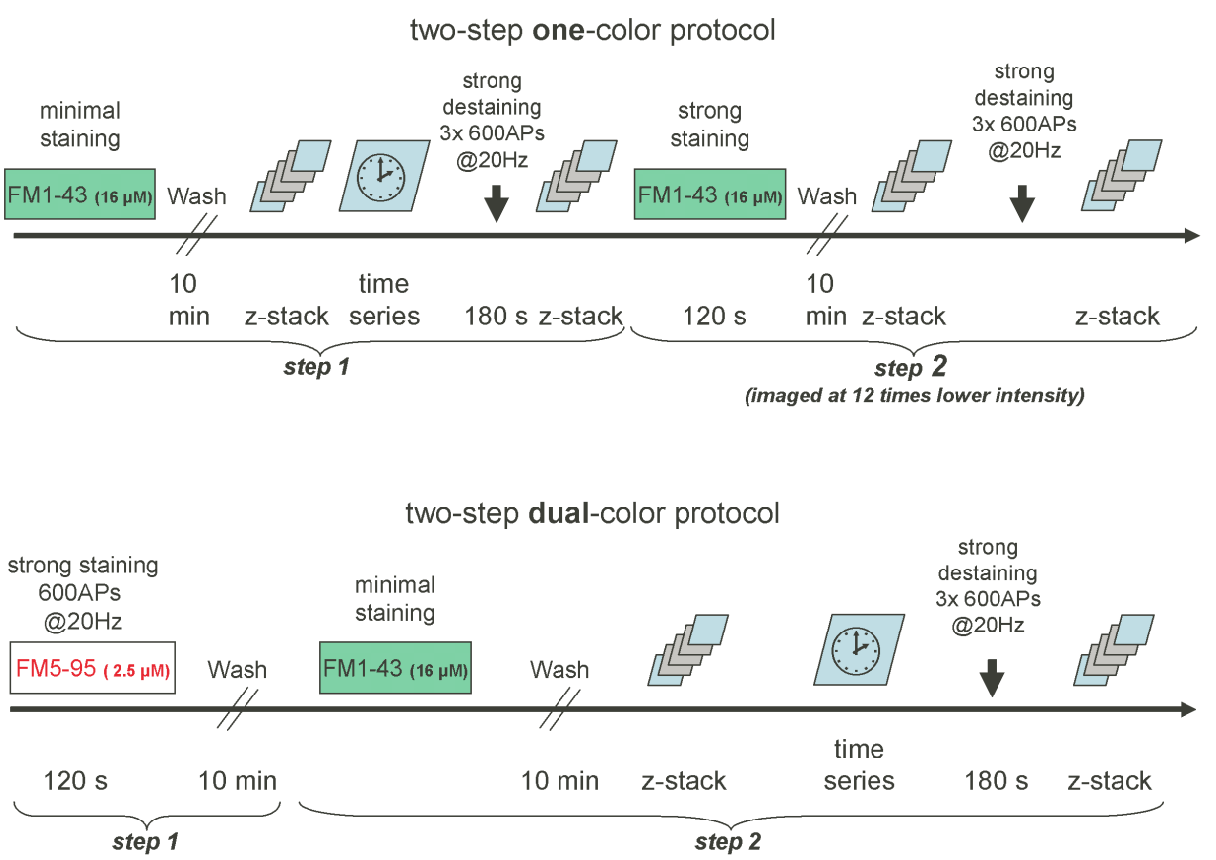

Figure 2.5: The upper panel shows the two-step one-color protocol. Minimal staining and strong staining are achieved using only one type of dye. The strong stained boutons are imaged at 12 times lower intensity. The lower panel shows the two step dual-color protocol. The minimal and strong staining of the synapse are achieved using different dyes at different concentrations. 
Figure 2.6: Fluorescence emission spectra of FM 1-43 and FM 5-95: Synapses were stained with either dye and the spectrum was acquired using the $\lambda$ scanning mode of the Leica microscope. As explained in the caption of figure 2.7, a dichroic mirror was used for spectral separation when a culture stained with both dyes was imaged. An emission filter allowed to collect light between $\lambda=$ $510-560 \mathrm{~nm}$ in the green channel $(\sim$ $50 \%$ of total) and $\lambda>630 \mathrm{~nm}$ in the red channel. The bleed-through into the green channel was less than $3 \%$ and less than $15 \%$ in the red channel.

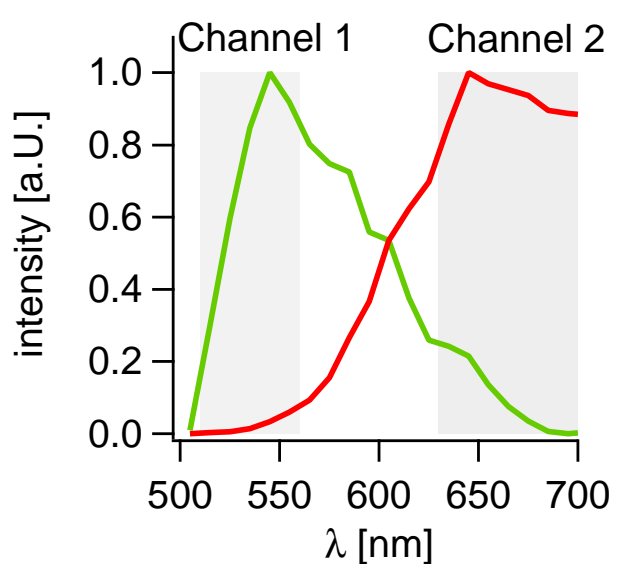

\subsubsection{Laser scanning microscopy of single synaptic vesicles}

Imaging single stained vesicles involves problems arising from autofluorescence and nonspecific labelling of cells, especially because the neurons grow on an approximately $5 \mu \mathrm{m}$ thick layer of astrocytes. Laser scanning in combination with either two-photon or confocal microscopy was ideally suited to overcome these problems. For this purpose, a standard confocal laser scanning microscope (Leica TCS SP2, Wetzlar, Germany) was used on a custom-made setup employing highly sensitive single photon counting modules, see figure 2.7 .

The microscope system allowed for two different laser excitation- fluorescence emission configurations: if a two-photon laser was used for excitation, the emission light of the fluorescence sample was collected closely behind the objective (at the nondescan port) with one detector (see figure 2.7 for details). If an argon laser was used for excitation, the emission signal was reflected back onto the optical axes of the excitation path passing the scanning mirrors (descan port) and directed to an external detector unit allowing separate detection of the green and red fluorescent light (see figure 2.7). For this dual-color detection unit, emission filters were chosen to maximize the collection of green fluorescent light by having only a minor bleed-through of red fluorescent light into this detection channel and vice versa. Refer to figure 2.6 for details on emission filters used and spectra of FM 1-43 and FM 5-95. 


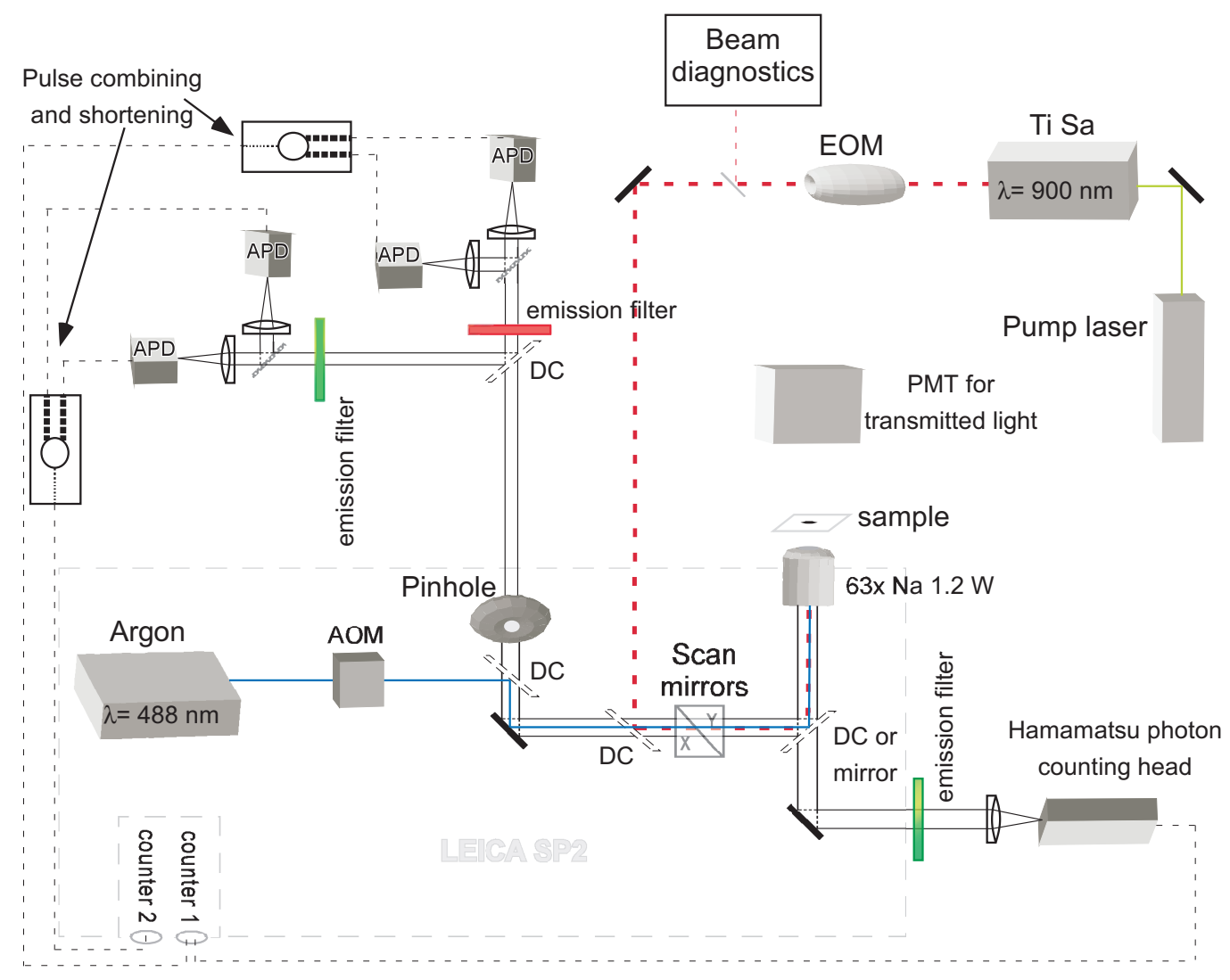

Figure 2.7: The basis for the setup was the Leica TCS SP2 confocal laser scanning microscope equipped with a $63 \times 1.2 \mathrm{NA}$ water objective. If the setup was operated in two photon counting mode, pulsed light from a titanium saphire laser (Spectra Physics, Darmstadt, Germany) was coupled into the optical beam path of the scanning mirrors via a dichroic (DC). A Pockels cell (electro-optic modulator, EOM; Polytek, Waldbronn, Germany,) allowed for fast shuttering of the laser. A small fraction of the laser light used for beam diagnostics (laser power, pulse width) was coupled out using a small glass plate in the beam path (for details see Tan et al. (1999)). Typically in two-photon mode the fluorescence light was coupled out to the nondescan port passing a BG 39 filter (Schott, Mainz, Germany) and focused on a Hamamatsu photon counting module (H7421-40, Hamamatsu, Herrsching am Ammersee, Germany) with a achromatic doublet lens with $f_{n}=70 \mathrm{~mm}$. Alternatively to two-photon excitation, single-photon excitation with a standard argon laser $\lambda=488 \mathrm{~nm}$ was possible. Fast shuttering of this light source was achieved with an acusto-optic modulator (AOM) integrated in the Leica system. In single-photon excitation mode, the emission light was directed back across the scan mirrors (descan mode) and passed a pinhole which was set to $1.70 \mathrm{AU}$. The emission light was split into green and red fluorescence light using a 610 DCXR dichroic (AHF, Tuebingen, Germany) and then passed emission filters (for details see figure 2.6). In each channel, the light was split with a half-transmitting mirror and focused onto APD's with achromatic doublet lenses $f_{n}=70 \mathrm{~mm}$. The two APDs on the green channel were type SPCM-AQR-13 (Perkin Elmer, Rodgau, Germany) and the two APDs on the red channel were type (SPCM-AQ-231, EG\&G, Vaudreuil, Canada). The TTL pulses from the APDs were shortened to $6 \mathrm{~ns}$ and the pulses from each two APDs belonging to the same color channel were combined using a custom-made device from PicoQuant (Berlin, Germany) and then counted inside the Leica SP2. Further optical components inside the microscope, such as other prisms and lenses, are not depicted for better clarity. A photo-multiplier tube (PMT) behind the sample allowed for the generation of Nomarski images. 


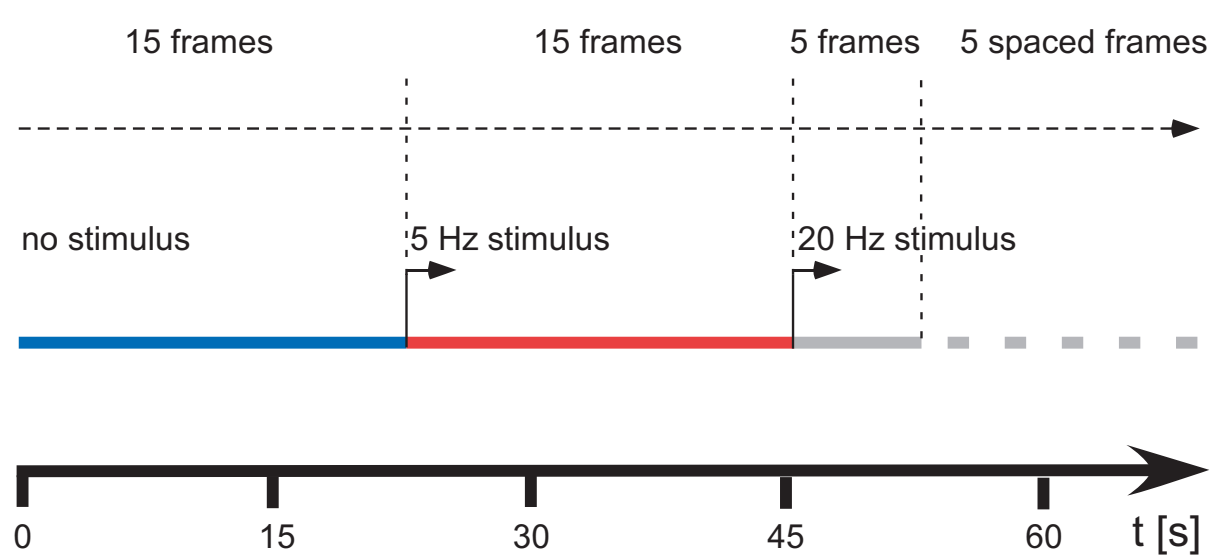

Figure 2.8: A time series was acquired using bidirectional laser scanning $(400 \mathrm{~Hz})$ at a pixel dwell time of $0.5 \mu \mathrm{s}$ and a total frame acquisition time of $1512 \mathrm{~ms}$. First, 15 images of the resting culture were acquired. During the next 15 images (frames 16-30) the culture was continuously stimulated at $5 \mathrm{~Hz}$ for a total of 75 APs. Starting at the beginning of frame 31, the stimulus frequency was increased to $20 \mathrm{~Hz}$. Images 36 to 40 were acquired with a lag time of $1512 \mathrm{~ms}$ between them.

\section{Image acquisition}

If not stated differently in the text, image acquisition parameters in experiments using argon laser excitation were as follows: The pixel size was $58.129 \mathrm{~nm}$ and the pixel dwell time was $0.5 \mu \mathrm{s}$ scanned bidirectionally in time series experiments (fast acquisition mode). The intensity of the argon laser was kept constant in all experiments with $4.5 \mu \mathrm{W}$ measured at the back pupil of the objective. Bleaching of FM 1-43 (taken up in synapses) in the fast acquisition mode was determined to have a half time of $t_{\frac{1}{2}}=122$ frames, and this was corrected for in the data analysis assuming bleaching to be mono-exponential. In z-stack recordings the pixel dwell time was $4 \mu \mathrm{s}$.

\section{Stimulation protocol to monitor vesicles during stimulation of the culture}

One aim of this thesis was to monitor single vesicles in real-time during resting conditions and during stimulation of the synapse. To achieve this, simultaneously to the acquisition of the time series in the two-step dual-color protocol, a stimulation protocol outlined in figure 2.8 was applied. 


\section{Computational methods and simulations}

\subsection{Monte-Carlo simulation of confocal spot detection data}

In the fluorescence fluctuation spectroscopy (FFS) experiments described by Jordan (2000), a standard confocal microscope was used to focus a laser spot onto single hippocampal synapses. Vesicles in the presynapse were labelled with fluorescent styryl dye and their movement led to fluctuations in the detected fluorescence signal. The following section will explain what information was already available from those experiments and which information was missing to simulate the complete FFS experiments. The aim was to specify the type of vesicle mobility that describes the experimental data measured in the FFS experiments. The results from these simulations, together with additional experimental data, are given in section 4.1.1.

\subsubsection{What does one need to know to simulate FFS experiments?}

1. The geometry of the detection volume: In the confocal microscope (Zeiss Confocor 1, Oberkochen, Germany) used by Jordan (2000), the detection volume was best described by a three dimensional Gaussian profile. The geometry of this volume was determined from calibration measurements and was found to extend considerably along the optical axes out of the small hippocampal synapse if centered on the synapse (average bouton diameter $\varnothing=1 \mu \mathrm{m}$, for details see Jordan (2000) ). It was shown that in such systems fluorescence fluctuations of particle movement along the optical axes can be neglected and that such a detection volume can be approximated to have the form of a cylinder (Gennerich and Schild, 2000), thus the illumination profile along the optical axis (in z-direction) is roughly constant, while in the xy-plane the illumination 
profile is best described by a 2D Gaussian. As a consequence only movements of vesicles in $\mathrm{x}$ - or $\mathrm{y}$-direction cause fluctuations in the detected fluorescence intensity. It was thus legitimate to simplify the problem from a $3 \mathrm{D}$ geometry to a 2D geometry. This corresponds to a projection of the approximately three dimensional spherical synapse $(\varnothing \sim 1 \mu \mathrm{m})$ onto a two-dimensional circle with an area of $3.14 \mu m^{2}(\varnothing \sim 1 \mu m)$. The same holds true for the detection volume, which is projected to the detection area (2D Gaussian disk).

2. The number of stained vesicles in the synapse: This number was not determined by Jordan (2000). In section 4.1.1 this number will be determined to be 24 vesicles in average per synapse using fluorescent imaging techniques of single dye stained vesicles and strong stained synapses (application of the two-step one-color protocol, see methods section 2.5 for details).

3. The brightness of each individual vesicle: Jordan (2000) estimated directly the brightness of a single vesicle from the FFS experiments. This approach was however, not very reliable. Thus this value was again estimated using a combination of imaging and fluorescence correlation spectroscopy techniques. This is explained in detail in section 4.1.1.

4. The type of movement of the objects of interest: To determine the parameters of movement was the aim of the simulation. Different types of motion were simulated and compared with the experimental data (see section 4.1.1.

\subsubsection{Monte-Carlo simulation of FFS experiments}

Small vesicles were simulated as fluorescent point sources with a brightness determined from experiments in section 4.1.1.

24 vesicles were placed at random positions in the $2 \mathrm{D}$ projection of the synapse. The particles were allowed to undergo a random walk in a $2 \mathrm{D}$ grid, with $\mathrm{h}=1 \mathrm{~nm}$ the grid space constant, $p=\frac{D \cdot \tau}{h^{2}}$ being the probability to jump to the next grid point, D being the $2 \mathrm{D}$ diffusion coefficient, and $\tau=0.01 \mathrm{~ms}$ the time step of the simulation.

Since vesicle movement in hippocampal synapses is believed to be restricted, the random walk was further confined within a small cage of radius $\mathrm{r}$ (this parameter was varied between $r=12.5-200 \mathrm{~nm}$ and for simplicity a square cage was simulated with 


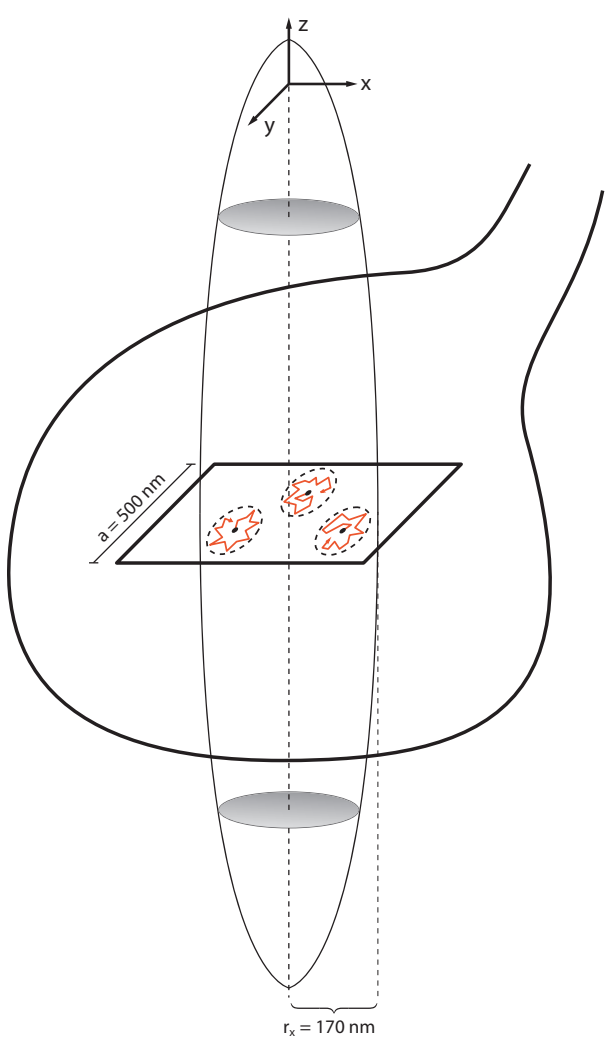

Figure 3.1: Schematic of a confocal laser volume centered on a synapse. Vesicles were allowed to undergo a random walk within a cage in a $2 \mathrm{D}$ disk. The relative dimensions of the confocal volume and the synapses approximate the true values as given in the text and described by Jordan (2000). Dashed ellipsoids indicate the cage and red lines the random walk of the vesicles (black dots).

edge length $\mathrm{r}$ ), and the object was reflected only if it reached the cage border.Please note that the type of movement simulated was an assumption, which was only legitimized when the simulation could indeed explain the experimental data (see results section 4.1).

Since only vesicles within the detection area contribute to the fluorescence signal (please note that the $e^{-2}$ radius of the detection profile in the experiments by Jordan (2000) was $\sim 0.17 \mu \mathrm{m}$ ) and only confined movement of vesicles was simulated, this properties could be used to enhance computation time of the simulation. Instead of simulating 24 vesicles in the projection of the synapse, only 8 vesicles where simulated in a three times smaller region (a square with edge length $\mathrm{a}=0.5 \mu \mathrm{m}$ ) that was centered on the detection area. Since vesicles at the edge of this region do not contribute anymore to the fluorescence signal, they could also cross the edge of the square when this happened during the random walk simulation. Figure 3.1 shows a schematic of the simulation.

Since the illumination profile was known, the intensity of each object was determined according to its spatial position in the 2D Gaussian disk relative to the center 
of the focal point. Intensities of all objects were integrated to yield the final fluorescence signal at each time point of the simulation. To simulate shot-noise (that is present in every experimental fluorescence signal), random numbers were drawn from a Poisson probability distribution with an expectation value given by the actual simulated fluorescence at each time point. Each simulation was performed to give a fluorescence trace of $120 \mathrm{~s}$ sampled at $10 \mathrm{~Hz}$ to match the experimental data (see Jordan (2000)). The data was analyzed similarly as described by Jordan (2000). In brief the fluorescence signal was high pass filtered with $0.05 \mathrm{~Hz}$ to remove slow frequency components. Jordan (2000) found this step necessary to obtain reproducible measurements. From the filtered data, the power spectra (PS) or the autocorrelation function $(\mathrm{ACF})$ were calculated as well as mean and variance of the fluorescence signal. Approximately 6-10 simulations of $120 \mathrm{~s}$ length were averaged. Error bars will be given as s.e.m..

\subsection{Particle tracking using non ideal photon detectors}

In ideal photon counting processes, every arrival of a photon on a detector can be counted. However, in this thesis, avalanche photo diodes were used as detectors, which exhibit a characteristic dead-time. This means that after detection of a single photon, the detector cannot detect further events until the dead-time $t_{d}$ has passed. The aim of this section was the theoretical analysis of the dead-time effect on the localization accuracy of subresolution particles in fluorescence microscopy using various algorithms. This will be done using simulations of the photon counting process in a microscope for the two cases of the ideal photon counting process and for the dead-time affected photon counting process. This study was first restricted to the one-dimensional case to enhance the speed of the simulations. The conclusion of this section is then also confirmed using a simulation in two dimension.

\subsubsection{Detectors with dead-time}

If a photon hits an ideal detector, this photon is detected with a certain probability which is determined by the quantum efficiency of the detector. The ideal detector can always detect as many photons as arrive with the same probability. Standard photo multiplier tubes (PMT) or CCD cameras come close to being ideal detectors. In contrast, a good example of a detector with dead-time is an actively quenched avalanche photodiode (APD) from Perkin Elmer (e.g. model SPCM-AQR-13), which 
typically exhibits a characteristic dead-time of $50 \mathrm{~ns}$. These detectors can count only single photons at a time, and after such a counting event, the detector cannot detect any photons for $50 \mathrm{~ns}$. However, photons that arrive on the APD during that time do not paralyze the detector for another $50 \mathrm{~ns}$, which is why these detectors are also termed non-paralyzeable detectors with dead-time. The detector can thus count the next photon 50 ns after the last counting event, no matter what happens during deadtime.

The dead-time $\left(t_{d}\right)$ effect causes an apparent loss in detection efficiency if the photon flux (here given in $\frac{\text { photons }}{\mu s}$ ) is increased (Hopt and Neher, 2001). The number of actually detected photons $N_{t_{d}}^{\text {theory }}$ can be calculated from the number of photons $N_{\text {ideal }}^{\text {theory }}$ that are actually arriving on the detector (Fessler and Yu, 2000)

$$
N_{t_{d}}^{\text {theory }}=\frac{N_{\text {ideal }}^{\text {theory }}}{1+N_{\text {ideal }}^{\text {theory }} \cdot t_{d}} .
$$

For ideal detectors, the photon detection process is a point process following Poisson statistics. The probability distribution for the number of counting events $n$ in a time interval $\Delta t$ is thus given by

$$
\operatorname{Pr}^{\text {Poisson }}\left[n \text { events in }\left(t, t+\mu^{\prime} \Delta t\right)\right]=\frac{\left(\mu^{\prime} \Delta t\right)^{\cdot} e^{-\mu^{\prime} \Delta t}}{n !},
$$

with $\mu^{\prime}$ the mean photon flux $\left(\frac{\text { photons }}{\mu s}\right)$ within the time interval $\left(t, t+\mu^{\prime} \Delta t\right)$. In a counting process following Poisson statistics, the variance is identical to the mean number of detected photons. For the avalanche photo diodes, the counting statistics are not described any more by Poisson statistics due to the presence of dead-time $t_{d}$. The probability distribution for the number of counting events in a time interval $\Delta t$ of such a point process is given by (Hillesheim and Muller, 2003)

$$
\begin{aligned}
\operatorname{Pr}^{t_{d}}\left(n \text { events in }(t, t+\Delta t) ; t_{d}\right) & =\sum_{j=0}^{k=n} \cdot \frac{\mu \cdot\left(1-t_{d} \cdot k\right)^{j}}{j !} \\
& -\sum_{j=0}^{k=n-1} \cdot \frac{\left.\mu \cdot\left(1-(k-1) \cdot t_{d}\right)\right)^{j}}{j !}
\end{aligned}
$$

where for simplicity the time interval $\Delta t$ was fixed to $1 \mu \mathrm{s}$ and absorbed into the mean of the probability distribution with $\mu=\mu^{\prime} \cdot \Delta t$. The variance of the process 
Figure 3.2: Theoretical variance dependence from the mean photon counting rate for an ideal detector (dashed line) and for a detector with dead-time $t_{d}=50 \mathrm{~ns}$ (solid line).

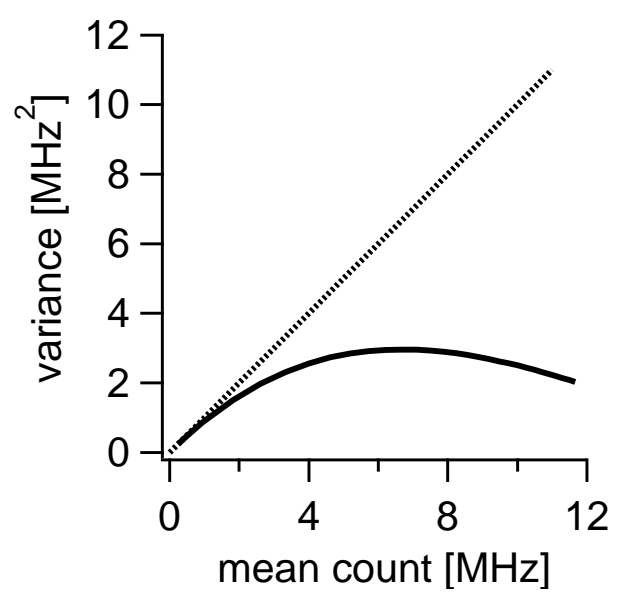

$v a r_{\text {detected }}$ for a given mean of photons arriving on the detector can be calculated by (Fessler and $\mathrm{Yu}, 2000$ )

$$
\operatorname{var}_{t_{d}}^{\text {theory }}=\frac{N_{\text {ideal }}^{\text {theory }}}{\left(1+N_{\text {ideal }}^{\text {theory }} \cdot t_{d}\right)^{3}} .
$$

Figure 3.2 shows a theoretical variance over mean plot for ideal and dead-time detectors. Please note that for the dead-time affected counting, the variance first increases and then decreases with the mean count rate.

\subsubsection{Distortion of the point spread function by non-ideal detection}

An imaged fluorescent subresolution particle appears as a point spread function (PSF), which is specific for each microscope. For high numerical aperture objectives (e.g. a water 1.2 NA objective as used in this thesis), the in-focus light can be well described by a two-dimensional Gaussian illumination profile in the xy-plane (Thompson et al. 2002). This causes a subresolution particle to be imaged as a Gaussian distribution. If the point spread function is now detected by a non-ideal detector, the Gaussian distribution will be changed due to the dead-time effect, which essentially causes a significant loss of photons at high photon fluxes and only a minor loss at very low rates, respectively. For the rest of this section amplitudes of PSFs and photon fluxes will be given in $\frac{\text { photons }}{\mu s}$, the quantum efficiency of all detectors will be assumed to be $100 \%$ and the dead-time for the non-ideal detector will be fixed to $t_{d}=50 \mathrm{~ns}$. Figure 3.3 shows the shape of a one-dimensional $P S F_{t_{d}}^{\text {theory }}$ for a non-ideal counting process. 


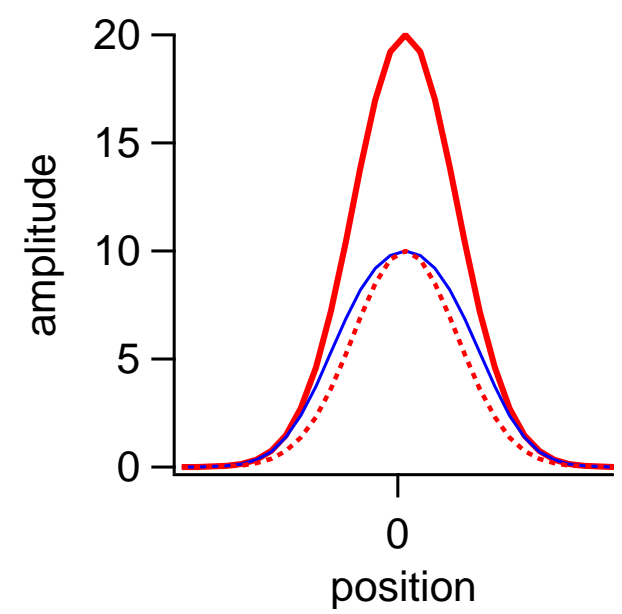

Figure 3.3: Shown in red is the 1D Gaussian $P S F_{\text {ideal }}^{\text {theory }}$ with amplitude $20 \frac{\text { photons }}{\mu s}$ and $\sigma=2.47$ pixel for the average of an ideal counting process (without shot noise). Overlaid in red is the $P S F_{t_{d}}^{\text {theory }}$ for a deadtime affected counting process with $t_{d}=50 \mathrm{~ns}$ (calculated using equation 3.1). For comparison, the ideal $P S F_{\text {ideal }}^{\text {theory }}$ with $\mathrm{A}=10 \frac{\text { photons }}{\mu \mathrm{s}}$ is shown in dashed red lines.

\subsubsection{Determining the center of a PSF}

In fluorescence microscopy, the center of the PSF determines the true position of the subresolution particle. As stated above, due to the stochastic nature of every photon detection process, a PSF detected ( $\left.P S F^{\text {detected }}\right)$ by a detector is not smooth anymore, but noisy due to shot-noise. Since shot-noise is a Poisson process, the "smoothness" of the $P S F^{\text {detected }}$ increases with the amplitude of the $P S F_{\text {ideal }}^{\text {theory }}$; additionally, the precision of most algorithms to determine the center of a $P S F^{\text {detected }}$ scales with the number of detected photons. Precision is usually defined as the standard deviation $\sigma_{\text {pos }}$ of the position estimate of any algorithm. In this thesis, typically the inverse variance will be analyzed, which is related to the precision by inverse variance $=$ $\frac{1}{\text { variance }}=\frac{1}{\sigma_{\text {pos }}^{2}}$. For a Gaussian $P S F_{\text {ideal }}^{\text {theory }}$ the theoretical maximal precision can be easily determined since each photon arriving on a detector can be regarded as a single measurement event with a Gaussian probability distribution. The best estimate of the center of the distribution is obtained by the average of the measured photon positions. Statistics predict the error of the mean to be the square root of the number of photons detected $\sqrt{N_{\text {photons }}}$. This results in

$$
\frac{1}{\sigma_{\text {pos }}^{2}}=\left(\frac{\sqrt{N_{\text {photons }}}}{\sigma_{P S F_{\text {ideal }}^{\text {theory }}}}\right)^{2},
$$

where $\sigma_{P S F_{\text {ideal }}^{\text {theor }}}^{2}$ is the variance of the spatial Gaussian PSF and $\sigma_{\text {pos }}$ is the standard deviation of the position measurement. For determining the position of a subresolution particle, three frequently used tracking algorithms are center of mass, direct Gaussian fit, and crosscorrelation (Cheezum et al., 2001; Thompson et al., 2002).

Direct Gaussian fit to the $P S F^{\text {detected }}$ is a computationally efficient way to determine 
the center of the $P S F^{\text {detected }}$. The following section will concentrate on the direct Gaussian fit. Even though Gaussian fit seems to be simple, some attention has to be paid to the fitting algorithm, i.e. mainly how the best fit is determined that gives the best unbiased model describing the original data. This is typically done using the method of Maximum Likelihood (Cramer (1958) and see appendix D.2 for a brief introduction to Maximum Likelihood estimators). The following paragraphs aim to answer some simple questions:

1. What is the impact of using "right" or "wrong" Maximum Likelihood (ML) estimators for a simple Gaussian fit.

2. What is the impact of photon loss for precision determination when working with non-ideal detectors.

3. How bad are these non-ideal detectors compared to ideal detectors for determining the position of a subresolution fluorescent particle in microscopy even at high photon fluxes.

Detectors such as APDs have a high quantum efficiency and employ almost no additional noise sources compared to e.g. photo multipliers tubes. However, they are not being used in most particle tracking experiments, which is most likely due to their non-linear photon detection behavior. The motivation for this work was to find out how severely the non-linear detection behavior jeopardizes the other advantages of these detectors in subresolution particle tracking studies.

\subsubsection{Simulation and position determination}

For determining the best fitting algorithm under various conditions, simulations were performed in the following way:

1. One dimensional Gaussian PSFs $\left(P S F_{\text {ideal }}^{\text {theory }}\right)$ were generated with a width $\sigma_{P S F}=$ 2.47 pixel and amplitudes $A$ ranging from 2 to $30 \frac{\text { photons }}{\mu s}$ according to

$$
f(x)=A_{\text {offset }}+A \cdot \exp \left(-\frac{1}{2} \cdot\left(\frac{x-p o s}{\sigma}\right)^{2}\right)
$$

and pos the center position of the Gaussian (here $A_{\text {offset }}$ was fixed to 0 ). 
2. The Gaussian $P S F_{\text {ideal }}^{\text {theory }}$ was generated 2000 times for each subpixel PSF position ranging from 0 to 0.9 in 0.1 steps. This was done to determine any eventual pixelation artefact of the fitting algorithms, which was not found for any presented algorithm.

3. Generating a detected PSF for an ideal detection process: The $P S F_{\text {ideal }}^{\text {deted }}$ was generated by drawing random numbers with the expectation value at every pixel given by the $P S F_{\text {ideal }}^{\text {theory }}$ from a Poisson distribution using the "DIPimage" (Delft Image processing group, TU-Delft, Netherlands) Poisson number generator in MATLAB (MathWorks GMBH, Ismaning, Germany).

\section{Generating a detected PSF for a dead-time biased detection process:}

The $P S F_{t_{d}}^{\text {detected }}$ was generated by drawing random numbers with the expectation value at every pixel given by the $P S F_{\text {ideal }}^{\text {theory }}$ using the self-programmed "dead-time" random number generator (see appendix D.1).

5. Each noisy $P S F^{\text {detected }}$ (either with dead-time noise, or with Poisson noise) was then fitted using the described fitting routines (see below).

\section{Ideal detectors ( $\left.P S F_{\text {ideal }}^{\text {detected }}\right)$}

First, the effect of different Maximum Likelihood (ML) estimators (see appendix D.2 for an overview) for the ideal detector was simulated, assuming only the presence of shot noise. In figure 3.4, the inverse variance is plotted as a measure of precision of the used algorithm over the amplitude of the simulated $P S F_{\text {ideal }}^{\text {theory }}$. One can see that a Poisson ML estimator gave the same result as theoretically expected from equation 3.5. This was also confirmed when a center of mass algorithm was used to compute the center $\left(c o i=\frac{\sum_{i}^{N} x_{i} I_{i}}{\sum_{i}^{N} I_{i}}\right.$ with $I_{i}$ being the intensity at pixel $x_{i}$ ), which yielded the same result. Since plotting the inverse variance over the amplitude of the simulated $P S F_{\text {ideal }}^{\text {theory }}$ resulted in a linear plot, the slope (intercept was 0 ) was defined as a quality factor $\delta$ for the goodness of the used fitting routine. The results are listed in table 3.1 . An ideal fit had $\delta_{\text {ideal }}^{\text {theory }}=\delta_{\text {ideal }}^{\text {coi }}=\delta_{\text {ideal }}^{\text {poi }}=1$. Furthermore, other ML estimators were also tested, for example the Gaussian ML estimator. Please note that this estimator was derived from Gaussian statistics (see equation D.4) and is not related to the shape of the PSF or the 1D Gaussian fit function itself. It is only a coincidence that the $P S F_{\text {ideal }}^{\text {theory }}$ in this simulation has the shape of a $1 \mathrm{D}$ Gaussian and that a Gaussian ML estimator was also tested. Using a Gaussian ML estimator for fitting the 1D Gaussian to the $P S F_{\text {ideal }}^{\text {detect }}$ resulted in $\delta_{\text {ideal }}^{\text {Gauss }}=0.88$ and using the simple chi square 
Figure 3.4: Simulated was the Gaussian $P S F_{\text {ideal }}^{\text {theory }}$ with increasing amplitude. The $P S F_{\text {ideal }}^{\text {theory }}$ was converted into a $P S F_{\text {ideal }}^{\text {deted }}$ by assuming an ideal counting process. Different ML estimators were tested to fit the $1 \mathrm{D}$ Gaussian to the $P S F_{\text {ideal }}^{\text {deted }}$. Shown is the result from these simulations in an inverse variance over simulated $P S F_{\text {ideal }}^{\text {theory }}$ amplitude plot. For reference, the theoretical precision is shown in red, which is partially overlapped by the results from the center of mass (coi) algorithm and the Poisson ML estimator, which were almost identical with the theory. The amplitude units are always given in $\frac{\text { photons }}{\mu s}$.

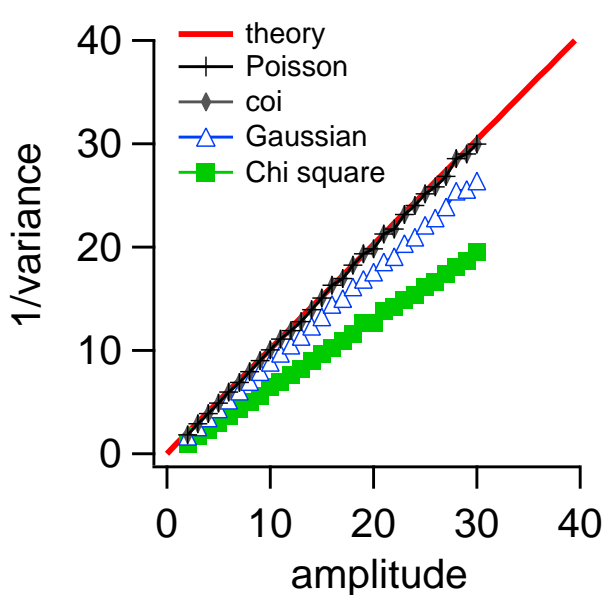

estimator, which is commonly used, gave only $\delta_{\text {ideal }}^{\chi^{2}}=0.64$ (see figure 3.4 and table 3.1 .

\section{Avalanche Photo diodes $\left(P S F_{t_{d}}^{\text {detected }}\right)$}

For the next step, the PSF detected by non-ideal counting processes $P S F_{t_{d}}^{\text {detected }}$ was simulated. The dead-time was fixed to $t_{d}=50 \mathrm{~ns}$. Figure 3.5 shows the results for fits with various ML estimators. The general appearance was that with higher amplitude of the theoretical ideal $P S F_{\text {ideal }}^{\text {theroy }}$, the plot is not linear anymore but curved. Please note that the integral of a Gaussian $P S F_{\text {ideal }}^{\text {theory }}$ scales linearly with the amplitude of the PSF. To allow easier quantification of the result, a line was fitted to the first 19 points (amplitude 2 to 20) to estimate the quality factor $\delta$. Please keep in mind that due to the slight curvature of the data the value for the quality factor (slope) $\delta$ is now underestimating the inverse variance for low amplitudes and slightly overestimating them for higher count rates (higher amplitudes). The results are also listed in table 3.1. Again, the Poisson ML estimator and the center of mass algorithm yielded identical results with $\delta_{\text {ideal }}^{c o i}=\delta_{\text {ideal }}^{p o i}=0.79$. The Gaussian ML estimator was not as good with $\delta_{t_{d}}^{\text {gauss }}=0.71$ and even worse was the $\chi^{2}$ estimator with $\delta_{t_{d}}^{\chi^{2}}=0.48$ (not shown in figure 3.5 but listed in table 3.1). The dead-time ML estimator (presented in appendix D.2 was also tested (see equation D.6). This ML estimator gave the best result with $\delta_{t_{d}}^{t_{d}}=0.80$. As this estimator was derived form the same function that is 

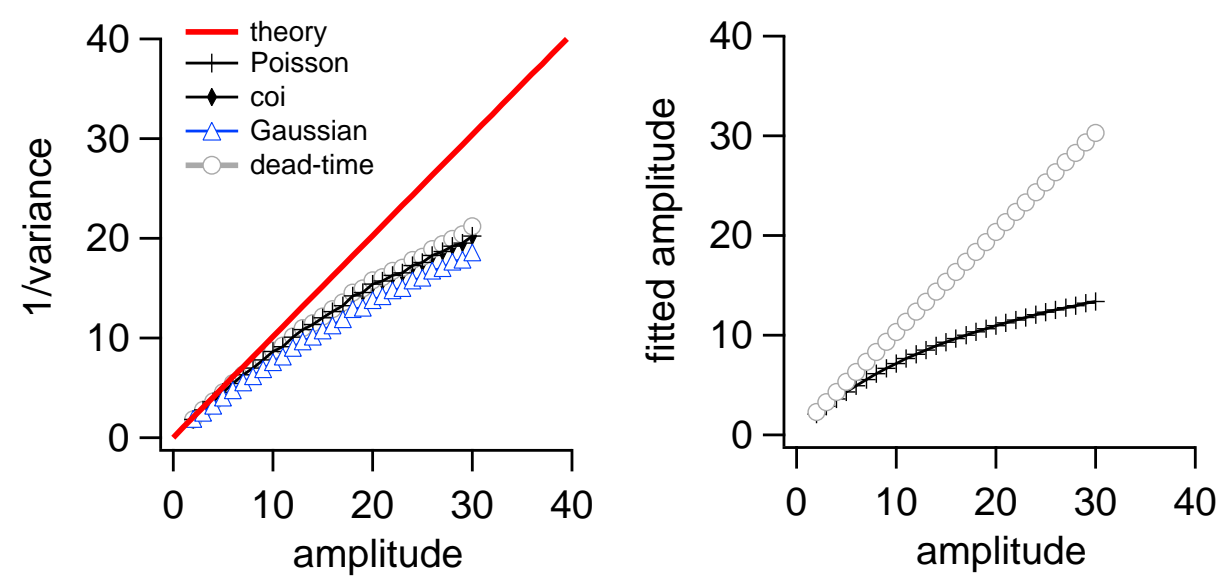

Figure 3.5: Simulation of the ideal Gaussian $P S F_{\text {ideal }}^{\text {theory }}$ that was converted into $P S F_{t_{d}}^{\text {detected }}$ assuming that the counting process is a dead-time biased point process (see equation 3.3). Different ML estimators were tested to fit a 1D Gaussian to the $P S F_{t_{d}}^{\text {detected }}$. The left panel shows that the dead-time ML estimator gave the best precision very similar to the standard Poisson ML estimator. The theoretical optimum for an ideal detector is shown as a reference in red. The right panel shows the fitted amplitude of the dead-time ML estimator in grey circles, which matched the simulated amplitude $P S F_{\text {ideal }}^{\text {theory }}$ and the result from the amplitude fit using the Poisson ML in black crosshairs.

analytically describing the photon counting process in the presence of dead-time, this was expected. Since the counting process cannot be described anymore as a Poisson process due to the presence of dead-time, it was a bit surprising that the performance (for determining the position of the $P S F_{t_{d}}^{\text {detected }}$ ) of the Poisson ML estimator was very similar to the dead-time ML estimator. Figure 3.5 shows that while the dead-time ML estimator recovered the amplitude of the theoretical ideal $P S F_{\text {ideal }}^{\text {theory }}$, the Poisson ML-estimator did not do so. However, this did not severely influence the quality of the position determination of the Poisson ML.

For completeness, other ML estimators were also tested. In appendix D.2 a modified Gaussian and Poisson ML estimator are presented where the mean and/or variance of the fitted Gaussian where converted into the respective dead-time affected mean and/or variances using equation 3.1 and 3.4 before calculating the Maximum Likelihood of the model. The modified Gaussian ML estimator (see equation D.7 in the appendix for details) yielded $\delta_{t_{d}}^{\text {Gauss }-P S F}=0.75$, which was slightly better than the standard Gaussian ML-estimator $\delta_{t_{d}}^{\text {Gauss }}=0.71$ (graph not shown, but see table 3.1 ). The precision of a modified Poisson ML-estimator (see equation D.8 in the appendix) was lower than the standard Poisson estimator with $\delta_{t_{d}}^{p o i-P S F}$ (graph not shown, but 
see table 3.1).

Another possibility was to correct the measured data for the dead-time effect. This is done by calculating the expected true number of photons that arrived on the detector from the number of counted photons per pixel, according to

$$
N_{\text {ideal }}^{\text {theory }}=\frac{N_{t_{d}}^{\text {detected }}}{1-N_{t_{d}}^{\text {detected }} \cdot t_{d}}
$$

The Gaussian fit was then performed on this corrected data. For all tested ML estimators this resulted in a decrease of the precision as summarized in table 3.1 $\left(\delta_{t_{d}}^{\text {cor-coi }}=\delta_{t_{d}}^{\text {cor-poi }}=0.76, \delta_{t_{d}}^{\text {cor-gauss }}=0.66\right)$.

\subsubsection{How severe is the loss of photons due to dead-time?}

When using non-ideal detectors, photons are lost if they arrive on the detector during dead-time. The main consequence is that less photons are collected, and this effect becomes more severe at higher photon fluxes. The integral of $P S F_{\text {ideal }}^{\text {theory }}$ with amplitude 20 and width of $\sigma_{P S F}=2.47$ is $124 \frac{\text { photons }}{\mu s}$. If detected on a detector with $t_{d}=50 \mathrm{~ns}$ the maximal amplitude of the measured $P S F_{t_{d}}^{\text {theory }}$ is $10 \frac{\text { photons }}{\mu s}(50 \%$ less $)$ and the integral $71 \frac{\text { photons }}{\mu s}$, which is $\sim 40 \%$ less. Since the inverse variance scales linearly with the number of collected photons (for an ideal detection process, equation 3.5) one would expect the inverse variance to drop by $\gtrsim 40 \%$, not taking into account that the dead-time affected $P S F_{t_{d}}^{\text {theory }}$ was also distorted and thus expected to further decrease the precision. However, photons are not simply lost, as they would be if one were to put an optical density filter into the emission light. The photons are lost in a known way as a consequence of dead-time, which is a constant characteristic of any given detector. This loss causes not only a decrease in the mean counting rate, but also a quenched variance (compare to figure 3.2). The simulations showed that the "right" ML estimator can make use out of this additional information and give a better estimate of the position determination, thus increasing the inverse variance. Figure 3.6 summarizes this effect for the dead-time ML estimator. It can be concluded that in dead-time affected photon counting processes, the contribution of a single measured photon at high photon fluxes was more than as expected by equation 3.5. For example, at amplitudes of the ideal $P S F_{\text {ideal }}^{\text {theory }}$ of $20 \frac{\text { photons }}{\mu s}$, the amplitude of the dead-time affected $P S F_{t_{d}}^{\text {theory }}$ was only half. However, the inverse variance was still $36 \%$ better 


\begin{tabular}{|c|c|c|c|c|c|}
\hline ML estimator used & detector model & $1 /$ variance & \multicolumn{3}{|c|}{ abbreviation } \\
\hline $\begin{array}{l}\text { theoretical preci- } \\
\text { sion (see equation } \\
3.5\end{array}$ & ideal & $1.0128 \pm 4.37 \mathrm{e}-11$ & $\delta_{\text {theory }}$ & $=$ & 1.01 \\
\hline center of mass & $P S F_{\text {ideal }}$ & $1.0018 \pm 0.00281$ & $\delta_{\text {ideal }}^{c o i}$ & $=$ & 1.00 \\
\hline Poisson & $P S F_{\text {ideal }}$ & $1.0018 \pm 0.00281$ & $\delta_{i d e a l}^{p o i}$ & $=$ & 1.00 \\
\hline Gauss & $P S F_{\text {ideal }}$ & $0.88151 \pm 0.00239$ & $\delta_{\text {ideal }}^{\text {Gauss }}$ & $=$ & 0.88 \\
\hline chi square & $P S F_{\text {ideal }}$ & $0.64159 \pm 0.00297$ & $\delta_{\text {ideal }}^{\chi^{2}}$ & $=$ & 0.64 \\
\hline center of mass & $P S F_{t_{d}}$ & $0.78873 \pm 0.00967$ & $\delta_{t_{d}}^{c o i}$ & $=$ & 0.79 \\
\hline Poisson & $P S F_{t_{d}}$ & $0.78873 \pm 0.00967$ & $\delta_{t_{d}}^{p o i}$ & $=$ & 0.79 \\
\hline Gauss & $P S F_{t_{d}}$ & $0.70728 \pm 0.0074$ & $\delta_{t_{d}}^{\text {Gauss }}$ & $=$ & 0.71 \\
\hline chi square & $P S F_{t_{d}}$ & $0.62596 \pm 0.00285$ & $\delta_{t_{d}}^{\chi^{2}}$ & $=$ & 0.48 \\
\hline$t_{d}$ Poisson & $P S F_{t_{d}}$ & $0.80427 \pm 0.00848$ & $\delta_{t_{d}}^{t_{d}}$ & $=$ & 0.80 \\
\hline $\begin{array}{l}\text { Poisson of shape } \\
\text { corrected } P S F_{t_{d}} \\
\text { (see equation } 3.1 \text { ) }\end{array}$ & $P S F_{t_{d}}$ & $0.74468 \pm 0.0127$ & $\delta_{t_{d}}^{p o i-P S F}$ & $=$ & 0.75 \\
\hline $\begin{array}{l}\text { Gauss } t_{d} \text { corrected } \\
\text { (see equation } 3.1 \\
3.4)\end{array}$ & $P S F_{t_{d}}$ & $0.74928 \pm 0.00798$ & $\delta_{t_{d}}^{\text {Gauss }-P S F}$ & $=$ & 0.75 \\
\hline $\begin{array}{l}\text { Poisson on } t_{d} \text { cor- } \\
\text { rected data }\end{array}$ & $P S F_{t_{d}}$ & $0.76435 \pm 0.0111$ & $\delta_{t_{d}}^{c o r-p o i}$ & $=$ & 0.76 \\
\hline $\begin{array}{l}\text { Gauss on } t_{d} \text { cor- } \\
\text { rected data }\end{array}$ & $P S F_{t_{d}}$ & $0.66436 \pm 0.0095$ & $\delta_{t_{d}}^{c o r-G a u s s}$ & $=$ & 0.66 \\
\hline $\begin{array}{l}\text { coi on } t_{d} \text { corrected } \\
\text { data }\end{array}$ & $P S F_{t_{d}}$ & $0.76435 \pm 0.0111$ & $\delta_{t_{d}}^{c o r-c o i}$ & $=$ & 0.76 \\
\hline
\end{tabular}

Table 3.1: Column one lists the type of ML estimator used for minimization, or whether the center of mass algorithm was used. Column two lists the type of noise that was used to generate a measured $P S F^{\text {detected }}$, which was either Poisson noise or dead-time affected noise (see appendix D.2). Column three lists the results from a line fit with the origin fixed to 0 , for points 2 up to an amplitude of $20 \frac{\text { photons }}{\mu s}$. Column four gives the abbreviation for the specific fit and simulation. In the last three rows, the raw data was corrected with equation 3.7 prior to the fitting routine. 
Figure 3.6: Shown in solid black is the expected inverse variance based on the number of photons detected by the non ideal detector (calculated using equation 3.5). Shown in black circles is the actual precision when using a dead-time ML estimator as simulated (compare to figure 3.5).

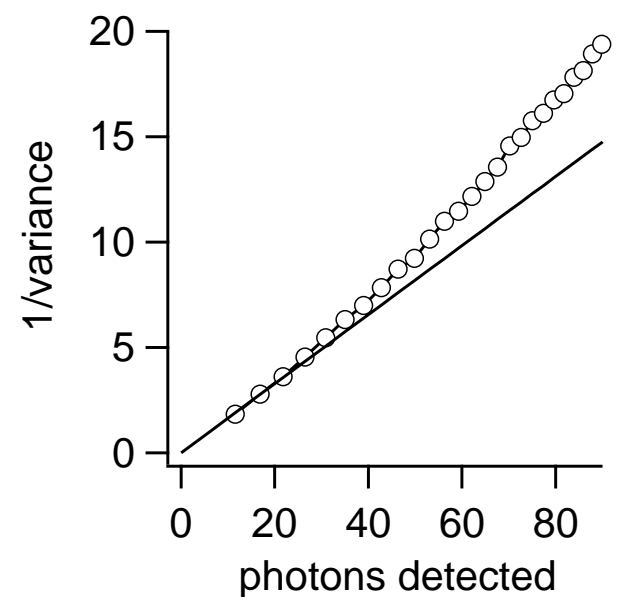

( $81 \%$ instead of $60 \%$ ) than expected from the integral of the $P S F_{t_{d}}^{\text {theory }}$.

Similar results were obtained if a simulation was performed where a constant background was assumed and a fit with four parameters was performed (see appendix E).

\subsubsection{Simulation of a 2D-Gaussian}

For a given amplitude $A$ of a 1D Gaussian for each function value of the Gaussian two data points have to be fitted (besides for the maximal amplitude). For a 2D Gaussian, the number of data points to be fitted increases with lower function values of the $2 \mathrm{D}$ Gaussian, due to the shape and the cylinder symmetry of the Gaussian. In dead-time affected photon counting, values for lower function values are also the one that are less biased due to dead-time. Since underlying principles of fitting a Gaussian to Gaussian shaped PSF are similar for the one and two dimensional case, it is expected that the effect described in the preceding paragraph for dead-time affected counting is also present for the $2 \mathrm{D}$ case, however, the strength of the effect might well be different.

To confirm the results of the $1 \mathrm{D}$ case, the simulations were repeated for the $2 \mathrm{D}$ case (this was done in IgorPro, with very similar routines written as described for the use in MATLAB). To save computation time for each amplitude of the Gaussian $A$ only 200 iterations of generating a $2 D-P S F^{\text {detected }}$ and fitting a 2D Gaussian to this PSF were performed. Due to the symmetry of the 2D Gaussian the variances for each $\mathrm{x}, \mathrm{y}$ position estimate are theoretically the same and were thus pooled together. Two simulations were performed. First, the $2 D-P S F_{\text {ideal }}^{\text {deted }}$ was fitted using the Poisson 


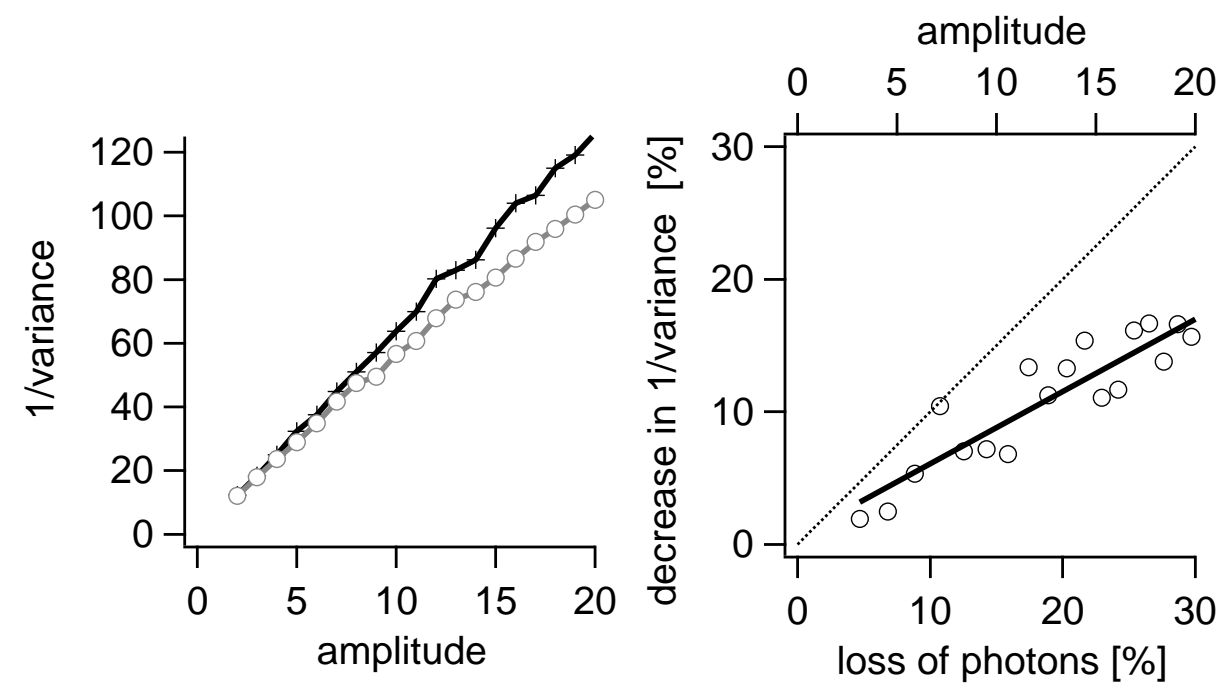

Figure 3.7: Shown is the inverse variance of the position estimate as a function of the $2 D-P S F_{\text {ideal }}^{\text {theor }}$, for the dead-time affected counting process and fit with the dead-time ML (grey circles) and for an ideal counting process and fit with the Poisson ML (black crosshairs). In the right panel for a given amplitude of the $2 D-P S F_{\text {ideal }}^{\text {theory }}$ (upper axis) the loss of photons due to the dead-time counting process was calculated. Plotted is the loss in inverse variance between the simulation for dead-time and ideal counting process over the loss of photons. The diagonal line is shown for better orientation. The solid black line is a line fit with intercept 0.65 and slope 0.54 .

ML estimator and the results from this simulation are plotted versus the amplitudes 2-20 $\frac{\text { photons }}{\mu s}$ of the $2 D-P S F_{\text {ideal }}^{\text {theory }}$ in figure 3.7. Second, the $2 D-P S F_{t_{d}}^{\text {detected }}$ was simulated and fitted using the above described dead-time ML estimator (see also equation D.6. The resulted inverse variance of the position estimate is also plotted versus the amplitude of the theoretical ideal $2 D-P S F_{\text {ideal }}^{\text {theory }}$ in this figure. The inverse variance for Poisson noise and fit with a Poisson estimator for an amplitude of $20 \frac{p h o t o n s}{\mu s}$ for a simulated amplitude of the $2 D-P S F_{\text {ideal }}^{\text {theory }}$ was $125.9 \frac{1}{p_{i x e l} l^{2}}$. The average number of photons detected (the integral) at this amplitude is $770 \frac{\text { photons }}{\mu s}$. For the simulation of a counting process with dead-time and fit using the dead-time ML estimator the inverse variance at an amplitude of the theoretical ideal $2 D-P S F_{\text {ideal }}^{\text {theory }}$ of $20 \frac{\text { photons }}{\mu s}$ was $105.1 \frac{1}{\text { pixel }^{2}}$. However, the integral of the average dead-time biased $2 D-P S F_{t_{d}}^{\text {theory }}$ is $534 \frac{\text { photons }}{\mu s}$. Thus due to the dead-time effect $30 \%$ less photons were detected relative to the ideal detection case, however, the inverse variance only dropped by $17 \%$. The right panel in figure 3.7 summarizes the loss in inverse variance versus the loss in photons for the dead-time detection process relative to the ideal detection process. 


\subsection{Simulation of single synaptic vesicle tracking}

One aim of this thesis was to develop a method to monitor single synaptic vesicle mobility inside hippocampal synapses (boutons) in real-time. Due to the small physical size of a hippocampal synapse and the limited resolution of a light microscope, this can only be done when one single vesicle per synapse is fluorescently labelled. It will be outlined in the results section 4.2 that in order to reliably track a single vesicle per synapse, it was in fact necessary to track both the vesicle and the corresponding synapse simultaneously. For this simultaneous tracking experiments the two-step dual-color protocol, introduced in figure 2.5, was used. The whole vesicle recycling pool of the synapse was first stained with a red fluorescent styryl dye, and the synapse was then minimally stained using a green fluorescent styryl dye yielding a small number of green vesicles per synapse. Before the actual off-line tracking of synapses and corresponding vesicles in a region of interest (ROI) can take place, those synapses that contain only one single green fluorescent vesicle had to be identified. This could be done by analyzing the intensity of a green fluorescent spot, as explained in detail in the methods section 2.3. However, particle tracking, especially in neuronal cultures, has several pitfalls (see below). To avoid these it was helpful to first simulate the dual-color simultaneous synapse and vesicle tracking protocol. These simulations will be outlined in the following sections, and an automated algorithm will be presented that allowed reproducible particle tracking experiments.

\subsubsection{Simulation of single vesicle and synapse movement}

A schematic of the simulation that is intended to reproduce the experimental data of the dual-color experiments is presented in figure 3.8. First, 2D images of a vesicle and a synapse were generated in separate channels, and allowed to undergo a random walk (for 1000 frames). To mimic the noise caused by autofluorescence and nonsynaptic staining in the experimental data, Gaussian noise was added. The generated images were then subjected to a tracking algorithm. The subresolution particle (a hippocampal single synaptic vesicle $\varnothing \sim 35 \mathrm{~nm}$ ) was fitted with a 2D Gaussian fit (using a $\chi^{2}$ estimator, see equation D.5

$$
\operatorname{Gauss}_{2 D}=A_{\text {offset }}+A \cdot \exp \left[-\frac{1}{2}\left(\frac{\left(x-x_{\text {pos }}\right)^{2}}{\sigma_{x y}^{2}}+\frac{\left(y-y_{p o s}\right)^{2}}{\sigma_{x y}^{2}}\right)\right],
$$

with $A_{\text {offset }}$ the offset of the amplitude $A$ due to background and $x_{\text {pos }}, y_{\text {pos }}$ the center of the Gaussian with width $\sigma_{x y}$. 
The results of this simulation are shown in figure 3.8. Additionally, the average width $\left(\sigma_{x y}\right)$, amplitude $(A)$ and the amplitude offset $\left(A_{\text {offset }}\right)$, as well as their standard deviations were determined from this simulation and are summarized in table 3.2 . The synapses, which can vary in size and shape, were tracked with a crosscorrelation algorithm (Heintzmann (1999), see also figure 3.8). This algorithm determines the shift of one image at a time point $t$ to a reference image at a different time point, which was fixed to frame 15 (in the experiments in section 4.3 this was the last frame where the culture was not stimulated).

\subsubsection{Selection criteria for single vesicle tracking}

For reproducible particle tracking experiments, the analysis of ROIs has to be limited to particles with similar intensity and similar signal-to-noise ratio. These requirements are accompanied by the first pitfalls:

1. The signal to noise ratio in a single image is not easily determined. A very good estimate of the signal to background ratio comes from the Gaussian fit to the fluorescent spot. For example, an object with low intensity would also yield a fit with low amplitude, and an exceedingly high background would yield an increased value of the amplitude offset.

2. The intensity of single vesicles during the acquisition of the time series is not necessarily constant. Since a stimulus protocol is applied simultaneously (see figure 2.8 in the methods section), vesicles can fuse and lose their dye content. If the vesicles lose their complete dye content upon fusion the tracking algorithm will usually fail (because the Gaussian fit cannot converge), and this is easily noticed by the algorithm. However, Aravanis et al. (2003b) suggested that single vesicles can only partially lose dye upon fusion. If a major portion of the dye is lost, but not all, the precision of the algorithm will decrease. This situation could mimic the effect of a higher mobility of the object. To rule out this possibility, it is not just sufficient to merely pick fluorescent puncta of single vesicles with similar intensity at the beginning of the time series, but it is also necessary to halt the algorithm and exclude objects that have lost a major fraction of their initial intensity during the time series. Aravanis et al. (2003b) determined that most vesicles lose $\gtrsim 50 \%$ of their dye content when they fuse, the threshold for halting the tracking algorithm was set to $60 \%$ of the original object amplitude. Here the original amplitude is defined as the average amplitude of the Gaussian 


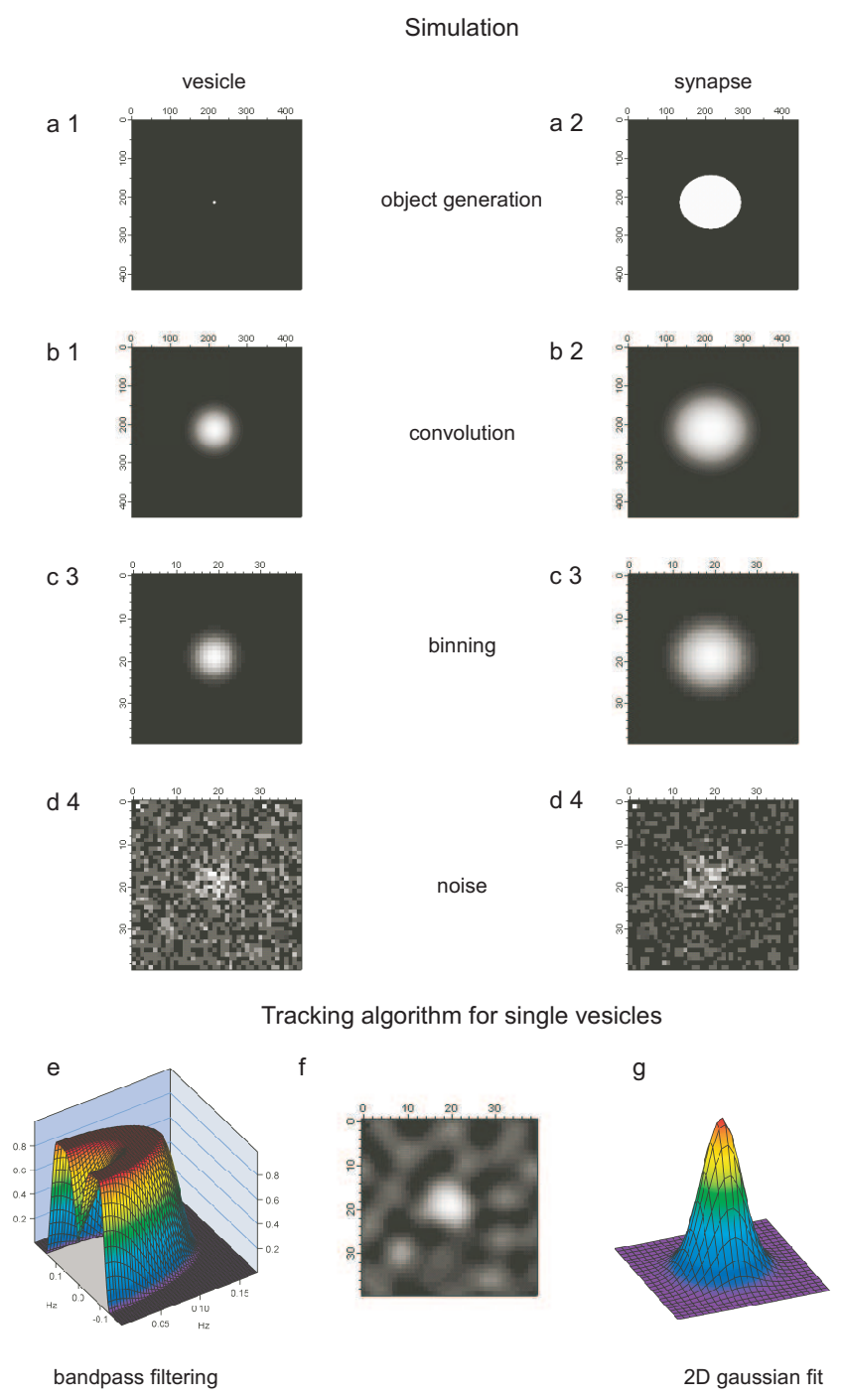

Figure 3.8: Simulation of simultaneous vesicle and synapse tracking. The left panel shows the simulation of a single vesicle and the right panel that of a corresponding synapse. In a 1, a vesicle with $\varnothing=37 \mathrm{~nm}$ was generated and in a $\mathbf{2}$ a corresponding synapse $\left(\varnothing_{x}=850 \mathrm{~nm}, \varnothing_{y}=745 \mathrm{~nm}\right)$ in a high resolution mask. These are typical sizes of vesicles and synapses in hippocampal cultures (Schikorski and Stevens, 1997). The objects were then convolved with the microscope-specific 2D PSF, i.e. with the diffraction image of a subresolution point source, $\mathbf{b} \mathbf{1}$ and $\mathbf{2}\left(\sigma_{P S F}=2.7\right.$, obtained from bead measurements, see table C.1 in the appendix for details). The objects were then allowed to undergo a random walk with $D_{\text {vesicle }}=5 \cdot 10^{-5} \frac{\mu m^{2}}{s}$ and $D_{\text {synapse }}=\frac{D_{\text {vesicle }}}{100}$ (for details on random walk simulation refer to section 3.1). In this step, also the bleedthrough of $15 \%$ from the vesicle to the synapse channel and about $3 \%$ in the other direction were introduced (see figure 2.6 for details on bleed-through). In c $\mathbf{1}$ and $\mathbf{2}$, the images were binned 11 times, to yield a final pixel size of $58 \mathrm{~nm}$. In the next step c 1 and 2 dead-time biased Poisson noise was simulated $\left(t_{d}=30 \mathrm{~ns}\right.$, see figure C.3 in the appendix for details, and section 3.2 .4 for simulating noise). Furthermore positive half Gaussian noise (to simulate background) with mean $=1$ in the vesicle channel and 0.5 in the synapse channel was added to each image. For single vesicle tracking, the single vesicle images were first filtered using a Hanning bandpass function e (with $\nu_{\text {high }}^{\text {corner }}=0.06 \mathrm{~Hz}$ and $\nu_{\text {low }}^{\text {corner }}=0.1 \mathrm{~Hz}$ ) to yield $\mathbf{f}$, and then fitted with a 2D Gaussian fit $\mathbf{g}$ (in a square ROI with 20 pixel edge length). The crosscorrelation of synapses was performed within square ROIs of 30 pixel edge length centered on the synapse. 

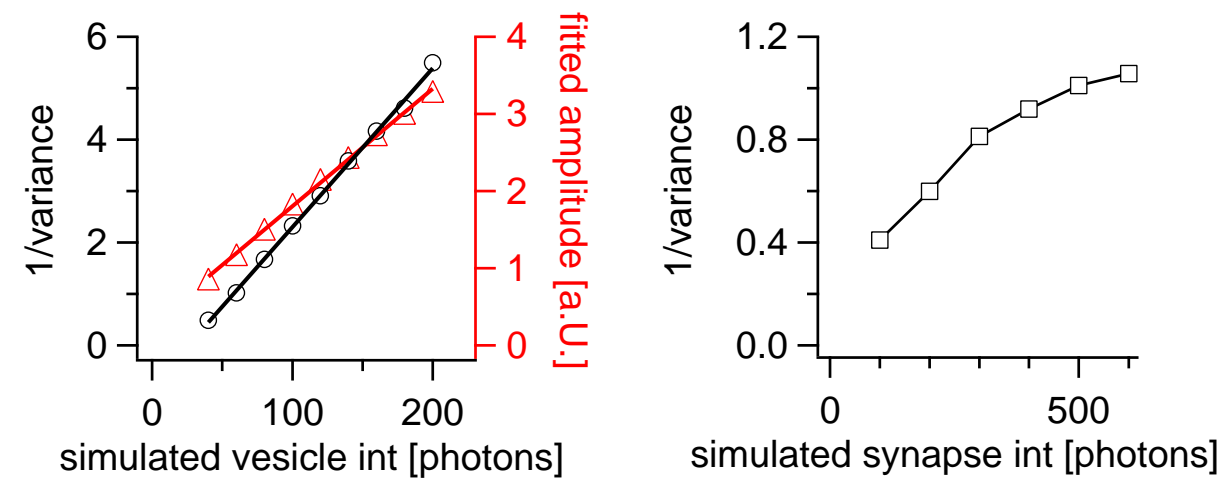

Figure 3.9: Results from simulating vesicle tracking experiments. The left panel illustrates the inverse variance (a measure of precision, see equation 3.5) of the Gaussian fit $\left(\frac{1}{\text { variance }}\right)$ as a function of the simulated vesicle intensity (black, left axes, line fit with $\frac{1}{\text { variance }}=-0.78+0.03 \cdot\left[\right.$ int $\left.\left._{\text {vesicle }}\right]\right)$. The fitted amplitude (red) also shows a linear dependence on the simulated intensity, and was fitted with a line fit yielding amplitude $=0.284+0.015 \cdot\left[\right.$ int $\left._{\text {vesicle }}\right]$. The right panel shows the inverse variance of the crosscorrelation algorithm vs the simulated synapse intensity.

\begin{tabular}{|c|c|c|}
\hline fit parameter & unit & estimate \\
\hline \hline position $_{\frac{1}{\text { variance }}}$ & pixel & $-0.78+0.03 \cdot\left[\right.$ int $\left._{\text {vesicle }}\right]$ \\
\hline amplitude & photon & $0.284+0.015 \cdot\left[\right.$ int $\left._{\text {vesicle }}\right]$ \\
\hline$c_{A}=\frac{\text { mean }_{A}}{\sigma_{A}}$ & -- & $0.1-0.25$ \\
\hline$\sigma_{\text {Gaussfit }}$ & pixel & $2.8 \pm 0.2$ \\
\hline$A_{\text {offset }}$ & photon & $0.21 \pm 0.02$ \\
\hline
\end{tabular}

Table 3.2: Summary of the accuracy of the vesicle fit position $\frac{1}{\text { variance }}$, the dependence of the fitted amplitude on the intensity (see figure 3.9), the range of the coefficient of variation for the amplitude fit $\left(c_{A}=\frac{m_{e a n}}{\sigma_{A}}\right)$, as well as mean and standard deviation of the fitted Gaussian width $\sigma_{\text {Gauss } i t}$ and the amplitude offset due to background $A_{\text {of fset }}$. The pixel dwell time in the simulation was fixed to $0.5 \mu \mathrm{s}$. All given photon counts refer to this time interval. 
fit of the single vesicle over all frames where the culture was at rest (frame 1 to 15 of the time series, see section 2.3.2.

As the bouton diameter was less than the diameter of the PSF in the axial (z-) direction (see caption of figure C.1 in appendix C for details), fluorescence changes of $>20 \%$ were very unlikely to be caused only by movement of the vesicle within the bouton. Therefore, in most cases a drop of more than $40 \%$ was assumed to be the consequence of a vesicle fusion. These events will accordingly be referred to as fusion or release events.

3. If a stained vesicle fuses, its dye content is released or partially released. Unfortunately, the FM 1-43 dye is not immediately washed away, but has a departitioning rate of $k_{\text {off }} \sim 3 s$ (Klingauf et al., 1998). Dye that was released from the vesicle but was not completely washed away from the ROI can lower the precision of the tracking algorithm. To avoid any bias due to this effect, the tracking algorithm was always halted two frames $(\sim 3 \mathrm{~s})$ before any detected fusion event.

In this thesis, single vesicle experiments were performed under a variety of different biological conditions. To allow for comparison of different experiments, consistent criteria to decide which vesicles were included in or excluded from analysis were needed. Defining these selection criteria is always a compromise between what is desirable in terms of optimizing the measurement precision and what is experimentally feasible. With this in mind (and taking into account the issues explained above) the following selection criteria were determined for all ROIs in all dual-color particle tracking studies. The criteria can be divided in those determined from the time series experiments and those determined from the z-stack recordings (for details on z-stack and time series recordings refer to figure 2.5 in the methods section).

1. Selection criteria from z-stack recordings.

a) Only single vesicles, as determined by the intensity histogram measurements (vesicles with intensities within 95\% confidence of the first peak, and intensity $>60$ photons in a square ROI of 11 pixel edge length, see for example figure 4.11 in the results section), were taken for analysis.

b) Only vesicles belonging to synapses with an intensity higher than 100 photons (within a square region, edge length $=19$ pixel) were tracked. To avoid 
bias of excessive bleed-through of the red channel into the green fluorescent channel, the upper limit of synapse intensity was set to $1.5 \times 19^{2}$ photons.

2. Selection criteria from time series recordings.

a) The simulation showed that the amplitude of the Gauss fit correlated linearly with the intensity of the simulated vesicle (see figure 3.9). Thus, the amplitude can be used directly as an estimate of the object intensity. Vesicles were only tracked if their average amplitude over the first 15 frames (where the culture was at rest) was larger than 1.4 photons, and the algorithm was halted if a long-lasting drop (for two consecutive frames) of $>40 \%$ of this value was found. In terms of analysis, the algorithm was actually halted two frames before such a strong amplitude drop, in order to avoid any bias due to the slow departitioning rate of FM 1-43 (see above).

b) The maximum offset allowed, $A_{\text {offset }}$ (a measure of background) was set to 0.3 photons.

c) The image of a subresolution particle is characterized by having a width comparable to that of the point-spread function of the microscope. $40 \mathrm{~nm}$ green fluorescent beads were allowed to settle on a neuronal cell layer and their width was determined by a Gaussian fit to be $\sigma_{x y}=2.8 \pm 0.4$. In single vesicle experiments, only those objects were tracked whose width in the fit never exceeded $\sigma=4.5$ (recall that the time series images were bandpass filtered, which increases the width of a the appearance of a subresolution particle).

d) From synapse tracking simulations, the average precision of the crosscorrelation algorithm was determined (see figure 3.9). In the experiments, it was only necessary to determine movement of the synapse. No shift correction was performed. The results from the crosscorrelation algorithm were low-pass filtered (using a Gaussian $5 \times 1$ kernel with width $\sigma=1.5$ ). If a movement exceeding 2 pixels was detected, the corresponding vesicle was removed from analysis. Please note that a shift of 2 pixels in a single frame did not necessarily mean that the object shifted by 2 pixels, but could also represent a deterioration of the tracking precision. The precision of the crosscorrelation algorithm was low (compared to the Gaussian fit of subresolution particles), and was additionally lowered by the fact that the intensity of synapses was changing during stimulation due to destaining. It 
is still possible that there is some residual synapse mobility as e.g. jittering that is not detected with the algorithm and those synapses and their corresponding vesicles are not removed from analysis. Such a possible minor synapse mobility can be seen as a contribution to lowering the precision limit of tracking vesicle positions in vivo and would thus be the same for all experimental conditions.

Two iterations of the algorithm As already stated, staining cultures with FM 1-43 using the minimal staining protocol results in low contrast images, while the images of strong stained synapses with the red fluorescent dye have a much higher contrast.

Not all regions where a synapse is identified in the red channel exhibits a clear spot (originating from one or more stained vesicles) or nothing (when no vesicles stained green) in the green channel. In fact several regions show a diffuse background staining or bigger fluorescent structures that would be spatially clipped when centering the ROI (square with 11 pixel edge length in the green channel) on them. Such regions had to be excluded from analysis. Typically this can be done by selecting only those regions where a "clear" fluorescent spot could be identified in a region with low background. In this thesis it was aimed to define clearly what is a "good" spot and what not and to control this selection procedure with the help of the algorithm. To achieve this, the experimentation first marked ROIs in the image independent of the brightness or background intensity, which was done by displaying the image with very few grey colors, such that pixels where a photon was detected appear as pixels with maximum brightness. The algorithm described in figure 3.10 was then applied to run a first iteration on this data. From the z-stack recordings the intensities measured before and after the destaining stimulus to yield the $\Delta F$ intensities, as described in section 2.3, were determined. In the time series described above, selection criteria were applied to all resting frames with only slight modifications. Only spots with an average fitted amplitude of $>1$ photon and a maximal offset of 0.3 photons were taken for analysis. This offset corresponds to $\sim 30$ photons within the ROI. This way it was ensured that $\Delta F$ intensities only from fluorescent spots having a sufficient signal to background ratio were plotted in the histogram. In the second iteration of the algorithm aforementioned selection criteria were applied to all frames in the time series until the detected release event for all those spots belonging to the first peak in the histogram.

The details of the tracking algorithm are also described in the flow chart in figure 
3.10 ,

In section 4.3 it will also be necessary to measure the average intensity in those regions where no vesicles were taken up during the minimal staining protocol. Again, regions where diffuse background staining or too large structures are visible have to be rejected from this analysis. Since in the preceding paragraph the maximal allowed amplitude offset due to background in a region of interest for a fluorescent spot was set to $\sim 30$ photons, in this analysis only those ROI's were analyzed where the intensity was less than twice that value, i.e. 60 photons. 


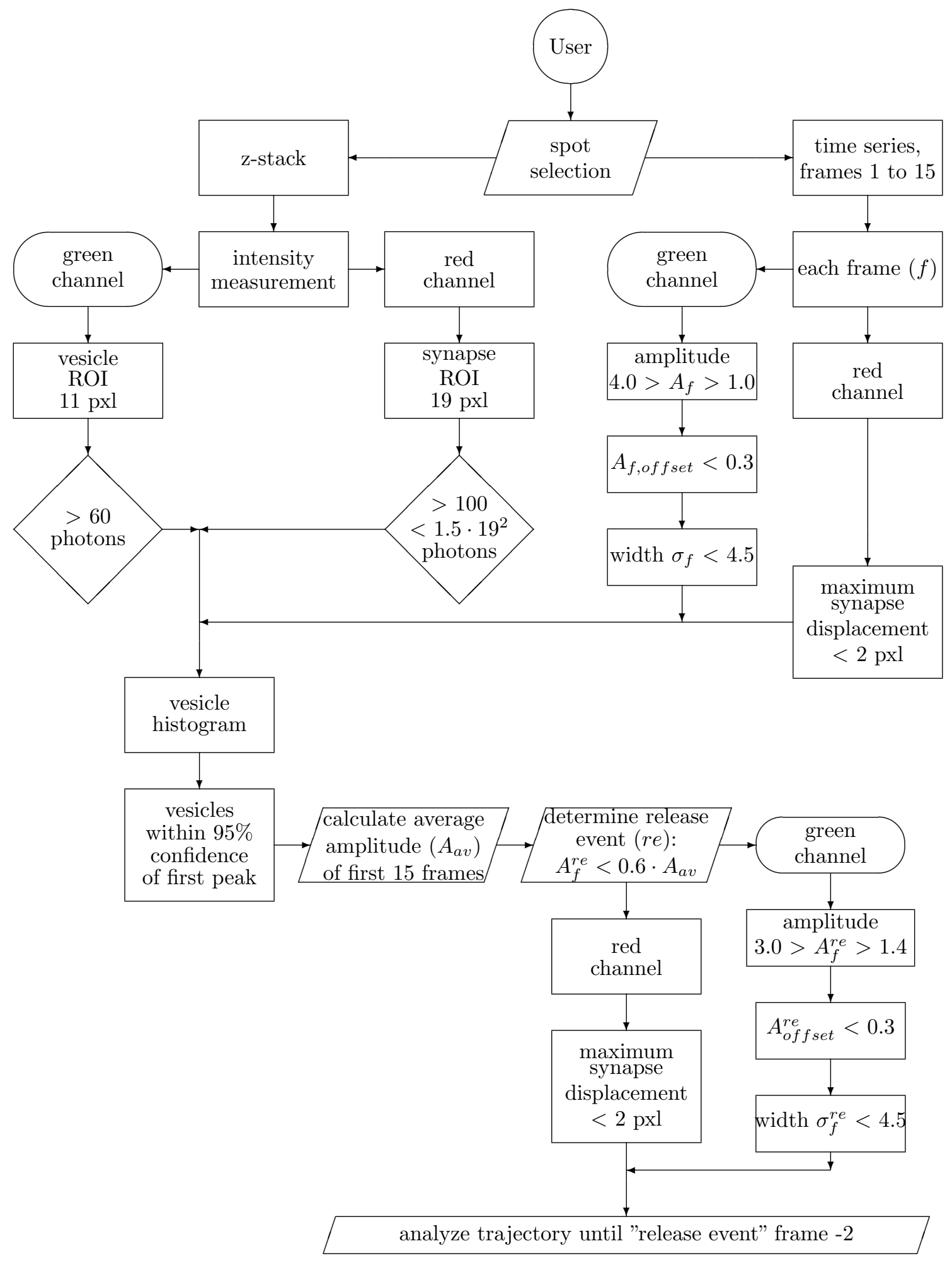

Figure 3.10: Flow chart for automated reproducible vesicle identification and tracking. The user only needs to select spot-like structures in the raw data, what follows is automated. The procedure was a two-step algorithm. In the first step, z-stack and time-series based selection criteria were used to identify those ROIs with a sufficient "imaging quality" (low background etc.). The $\Delta F$ intensities of fluorescent spots of these ROIs (before and after destaining stimulus, see methods section 2.3 for details) in the plane of best focus of the z-stack recording were then plotted in a histogram. Fluorescent spots within $95 \%$ of the first peak of this histogram were again subject to the time series selection criteria (second step), but this time for all frames (minus two) until the detected release event in the time series. 


\section{Results}

\subsection{Fluorescence fluctuation spectroscopy of vesicle mobility in synapses}

The study presented in this section aimed on extending the fluorescence fluctuation spectroscopy (FFS) study performed by Jordan (2000), who used a standard fluorescence confocal microscope to perform laser spot detection of individual FM 1-43 dye stained hippocampal synapses (boutons). Jordan (2000) recorded a fluorescence signal from an individual synapse for 120 seconds. These signals were analyzed using power spectra, autocorrelation, mean and variance analysis to unravel the origin of these fluctuations. In addition to studies of resting boutons, Jordan (2000) tested certain pharmacological treatments and their effects by analyzing the fluorescence signal. In the following section, experimental data from the FFS study performed by Jordan (2000) will be reanalyzed and summarized together with new experimental data, that allowed the simulation of the FFS experiment and deducing a quantitative model of vesicle mobility. A crucial point for the simulation was to have a reliable estimate of the fluorescence intensity contribution from a single dye stained vesicle in the FFS experiments. An approach will be presented using a combination of fluorescence imaging and fluorescence correlation spectroscopy to fulfill this task.

In the experiments performed by Jordan (2000) fluctuations of the fluorescence signal had two origins:

1. The photon detection process introduced shot-noise to the fluorescence signal. Since this is a Poisson point process, the variance is equal to the mean. This allowed for an easy determination of the variance contribution from shot-noise Qian and Elson, 1990b a). All values given in the following paragraphs for the variance are already shot-noise corrected variances, which were calculated by subtracting the mean from the variance of the fluorescence signal as described by Jordan (2000). This was done after high pass filtering of the fluorescence 
Figure 4.1: Variance as a function of the mean fluorescence from 299 control synapses recorded by Jordan (2000) (black crosshairs) given in kilo counts per second (kcps). The data was fitted with a line yielding an intercept of $-0.02 \pm 0.27$ and a slope of $0.103 \pm 0.018$. The scatter in the plot depicts the high bouton-tobouton variability in these measurements. The grey squares show the result from a simulation of control cells (see chapter 4.1.2.

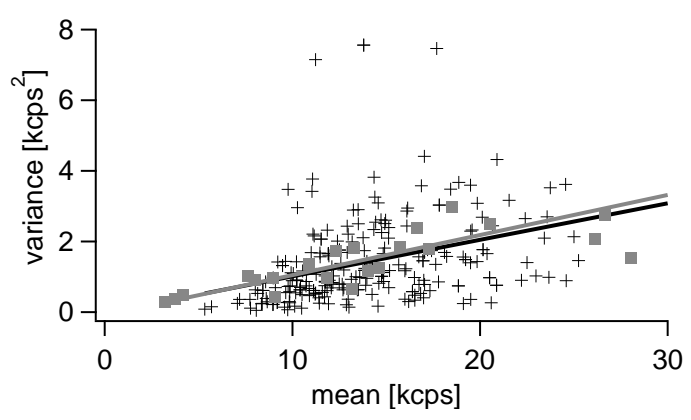

signal at $0.05 \mathrm{~Hz}$ (see Jordan (2000) and section 3.1 for details).

2. Additional variance was introduced to the fluorescence fluctuations when fluorescent objects moved in the detection volume. This variance depended critically on the movement and on the number of particles that move (see below).

Murthy and Stevens (1998); Aravanis et al. (2003b); Ryan et al. (1997) showed that single vesicle staining is quantal, i.e. all vesicles take up a similar amount of dye and appear in similar brightness. Thus the mean fluorescent signal of a synapse scales with the number of particles. Since every moving particle in the detection volume of the FFS experiments contributes to the variance, it is expected that the variance (after shot-noise correction) increases with the mean. In figure 4.1, all 229 control boutons measured by Jordan (2000) were reanalyzed and the plot shows that indeed the variance increased with the mean fluorescence.

The average number of fluorescent particles in the detection volume can either be obtained by fitting the amplitude of an autocorrelation function (Thompson, 1989), or directly by variance analysis (Magde et al., 1974) according to $\langle N\rangle=\frac{\text { mean }^{2}}{\text { variance.g }^{2}}$, with mean and variance estimated from the fluorescence signal and $g$ the geometry factor of the detection volume. A geometry factor is necessary to correct for the inhomogeneity of the detection volume. For details, the importance and pitfalls of the geometry factor refer to Kask et al. (1997). For the purpose of this thesis it is sufficient to recall that for a 3D Gaussian volume $g=\sqrt{8}$ and $g=2$ for a cylinder volume (Kask et al. 1997 ). However, mean and variance analysis on the data measured from Jordan (2000) returned typically a value of about $\gtrsim 50$ vesicles in the detection volume (see e.g. table 4.1). In the synapse of the hippocampal culture, it was expected that only about $\sim 30$ vesicles can be stained with FM dyes (for review 
see Harata et al. (2001). Since in the FFS experiments only about $10 \%$ of the total bouton volume were sampled within the small laser focus (Jordan, 2000), the number of $\gtrsim 50$ raised some doubts about the performed analysis, which was only valid if particles were assumed to freely diffuse.

To clarify this issue, the next aim was to get a reliable estimate of the brightness of a single vesicle in the confocal spot detection experiments.

\subsubsection{The brightness of a single vesicle in FFS experiments}

The brightness of one freely diffusing fluorescent species can be easily obtained by standard fluorescence correlation spectroscopy (Magde et al., 1972, 1974; Thompson, 1989). Unfortunately, dye stained vesicles are packed in a synapse and not available in a purified form. However, the brightness ratio of two subresolution particles is a constant in every fluorescence microscope, when the spectral properties of the microscopes are similar, which was taken care of in the performed experiments. Thus, the following approach could be used instead. The brightness ratio form vesicles and beads was accessible using imaging experiments. Since the brightness of small fluorescent beads was determined by Jordan (2000) under the same conditions as the other FFS experiments, the brightness of the vesicles in the FFS experiments could be calculated from the ratio (obtained from imaging experiments).

Brightness of beads in the FFS experiment Fluorescence fluctuation signals of bead solution acquired under the same conditions as all other FFS experiments were kindly made accessible by Randolf Jordan and Jurgen Klingauf. The fluorescence signal was subject to standard autocorrelation analysis and fit with a $3 \mathrm{D}$ one-component diffusion model (Thompson, 1989),

$$
f(\tau)=\frac{1}{N_{\text {eff }}} \cdot \frac{1}{\tau+\frac{\tau}{\tau_{\text {diff }}}} \cdot \sqrt{\frac{1}{\tau+s p^{2} \cdot \frac{\tau}{\tau_{d i f f}}}}
$$

with $\tau_{\text {diff }}$ the characteristic diffusion time, $N_{\text {eff }}$ the number of particles in the effective detection volume and $s p$ the structural parameter of the focus volume (for details see Jordan (2000)). The brightness of a single bead (cpb) was determined in this way to be $(1.57 \pm 0.02) \cdot \sqrt{8}$ kcps (kilo counts per second), as shown in figure 4.2. 
Figure 4.2: Autocorrelation function of a green fluorescent bead solution ( $\varnothing=40 \mathrm{~nm}$, bead solution measured by Jordan (2000) ) and a fit with a $3 \mathrm{D}$ diffusion model (equation 4.1.). $\tau_{\text {diff }}=165 \mathrm{~ms}$, amplitude $f(\tau)=1.12$, and the effective number of particles to be $N_{\text {eff }}=8.3$ and $s p=6.61$. These experiments determined the count rate per bead to be $c p b=(1.57 \pm 0.02) \cdot \sqrt{8} \mathrm{kcps}$.

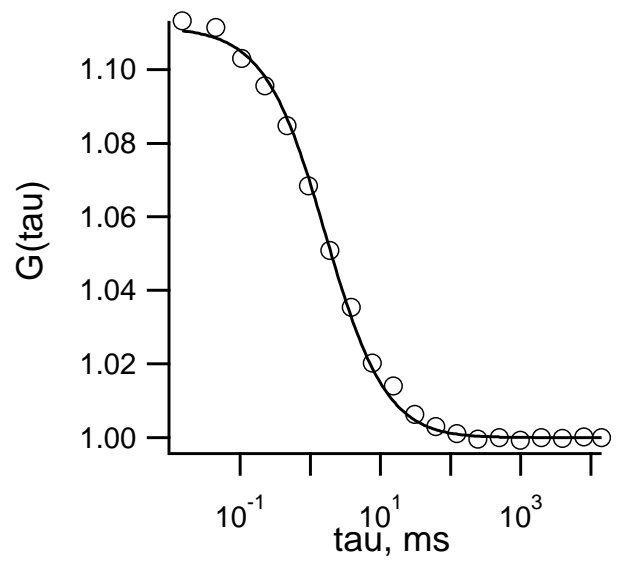

Brightness of single vesicles and beads in imaging experiments In order to determine the brightness ratio of vesicles and beads, an independent approach was carried out using laser scanning microcopy and application of the two-step one-color protocol (using argon laser excitation, refer to figure 2.5 for the details of the protocol).

Hippocampal cultures were first stimulated with only 5 APs at $5 \mathrm{~Hz}$ in the presence of $15 \mu M$ FM 1-43 applied for $10 \mathrm{~s}$ to achieve minimal staining of synapses. Only if a stimulation was performed during dye presence, fluorescence spots (with an appearance similar to those of $40 \mathrm{~nm}$ fluorescent beads) were visible after dye wash off, which were able to lose their fluorescence after arrival of a destaining stimulus. This was an indication that fluorescent spots originated from single vesicles or vesicle clusters. The $\Delta F$ intensities for these spots were determined by integration of the fluorescence intensity in a circular ROI centered on the spot $(\varnothing=13$ pixel $)$ and subtracting the image after arrival of the destaining stimulus from the one before. Figure 4.3 shows the $\Delta F$ intensity histogram of these fluorescent puncta, and the corresponding fit with equation 2.1 revealed the intensity of a single vesicle to be $\mu=96.8 \pm 3.2$ photons. If this value represents indeed the true fluorescence intensity contribution from a single vesicle, then the fluorescence intensity of a synapse where the complete recycling pools is stained should be at least $\gtrsim 30$ bigger than this value, to be in good agreement with the literature (Murthy and Stevens, 1998; Aravanis et al., 2003b; Ryan et al., 1997; Harata et al., 2001). To test this, the recycling pool was completely stained using a strong stimulus of $2 \times 600$ APs during 3 min application of FM 1-43. The average intensity of a bouton was found to be 52 times the value of a single vesicle (see figure 4.3), which encouraged confidence in the estimate of the fluorescence contribution from a single vesicle. Please note that the dye was present throughout the complete stimulus, so that any form of partial staining of vesicles was 
very unlikely. Furthermore, the average number of vesicles stained by the stimulus used in the FFS experiments by Jordan (2000) was determined to be 24 (120 AP's @ $10 \mathrm{~Hz}, 60 \mathrm{~s}$ dye application, see figure 4.3). The intensity of individual beads was now measured under the same imaging conditions and found to be $\mu=48.6 \pm 1.2$ photons (see figure 4.3). A vesicle was thus approximately two times brighter than a fluorescent bead and the brightness of a single synaptic vesicle in the FFS experiments was thus $c p v=2 \cdot 1.57 \cdot \sqrt{8}$ kcps.

With typical mean count rates of $10-15 \mathrm{kcps}$ of the fluorescence traces in the FFS experiments from Jordan (2000) (see table 4.1) this corresponds to $\sim 3$ vesicles in the detection volume rather than 50, proving the model of freely diffusing vesicles in the synapse to be wrong.

One explanation for the high number of particles determined by the above analysis could be that there was only a fraction of mobile particles $N_{m}$ diffusing on an immobile background. According to Qian and Elson (1990b), this fraction can be calculated using

$$
\frac{N_{m}}{N_{t}}=\frac{1}{c p v} \cdot g \cdot \frac{\text { variance }}{\text { mean }}
$$

with $N_{t}$ the total number of particles and $\mathrm{g}=2$ for a 2D Gaussian illumination profile (Kask et al. 1997). The results calculated according to this model for the experimental data from Jordan (2000) are listed in table 4.1. For control cells typically 2-3\% mobile components are found and one could rather expect to see bursts in the fluorescence trace in the rare event that a vesicle passes through the detection volume, which was not observed (see for example figure 4.5). Furthermore, no mobile component of freely diffusing vesicles was observed in fluorescence recovery after photobleaching experiments (FRAP) by Kraszewski et al. (1995). We can thus conclude that this model is also not sufficient to describe vesicle mobility in hippocampal boutons (see also discussion).

\subsubsection{Monte-Carlo simulations of FFS data}

The original data from Jordan (2000) was reanalyzed (all data was kindly made accessible by Randolf Jordan and Jurgen Klingauf). Changes in vesicle mobility were observed by Jordan (2000) when the myosin light chain kinase was inhibited and also 


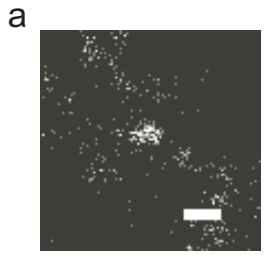

minimal staining

C

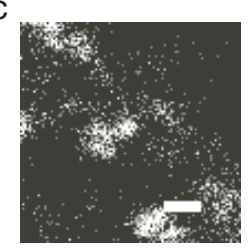

strong staining

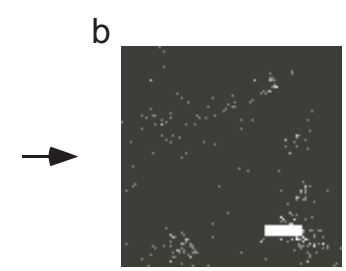

destaining

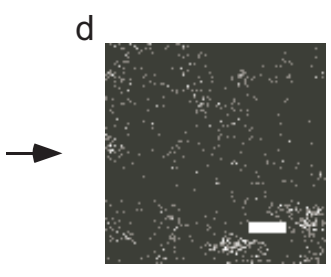

destaining

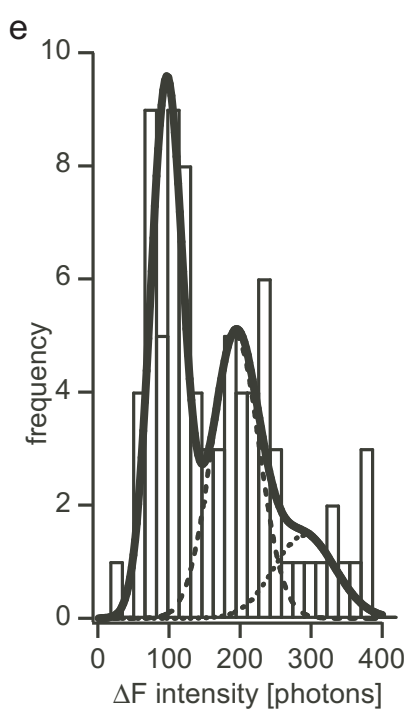

$\mathrm{h}$

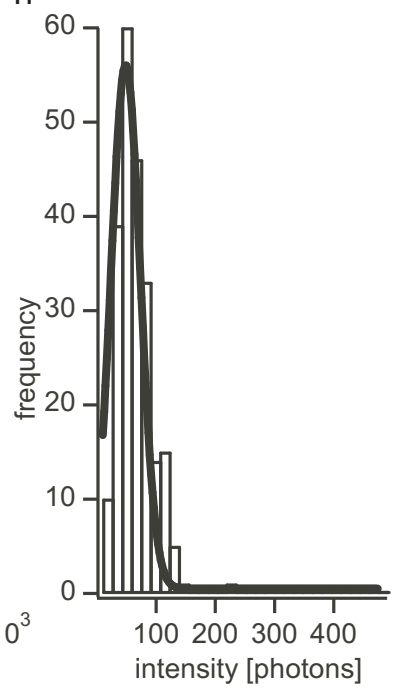

Figure 4.3: Brightness of single synaptic vesicles and beads. Panel a shows a region of interest of a minimally stained hippocampal culture (5 APs @ $5 \mathrm{~Hz}$ during $15 \mathrm{~s}$ of $15 \mu M$ FM 1-43 application). The fluorescence spot was lost upon further stimulation b. Functional synapses at this position are identified by a strong staining with dye and subsequent destaining $\mathbf{c}$ and $\mathbf{d}$. Please note that images $\mathbf{c}$ and $\mathbf{d}$ were acquired at 12 times lower intensity than $\mathbf{a}$ and $\mathbf{b}$. The intensity of individual spots in the minimally stained culture was measured by centering a circular region $(\varnothing=13 \mathrm{pxl})$ on it. The $\Delta F$ intensities (intensity difference before and after the destaining stimulus of the minimal staining protocol) are plotted in histogram e, yielding a quantal distribution which was fitted with equation 2.1 with $\mu_{\text {offset }}=0$ yielding the fluorescence of single vesicles to be $\mu_{\text {vesicle }}=96.8 \pm 3.2$ photons. Panel $\mathbf{f}$ shows the histogram of fully stained synapses $(2 \times 600 \mathrm{APs}$ and $90 \mathrm{~s}$ dye application) with a Gaussian fit centered at $5137 \pm 122$ photons, corresponding to 52 vesicles. Panel $\mathrm{g}$ shows the histogram of synapses stained under the same conditions as in the FFS experiment (120 AP's @ $10 \mathrm{~Hz}$ ), with a peak at $2353 \pm 206$ photons corresponding to 24 stained vesicles. In panel $\mathbf{h}$ the same beads that were used in the FFS experiments (see figure 4.2) were allowed to settle on hippocampal cultures and then imaged. The measured intensities within a circular region $(\varnothing=13$ pixel) were plotted yielding an absolute intensity of $\mu_{\text {bead }}=48.6 \pm 1.2$ photons. The emission filter used in the imaging experiments was similar to that used by Jordan (2000) $(\lambda=530-580 \mathrm{~nm}$ bandpass filter $)$. 


\begin{tabular}{|l|c|c||l|c||c|}
\hline drug & $\mathrm{n}$ & mean $[$ kcps $]$ & variance $[k c p s]^{2}$ & $\mathrm{p}$ & $\frac{N_{m}}{N_{t}}$ \\
\hline \hline $15 \mu M$ ML-7 & 14 & $9.98 \pm 1.09$ & $0.54 \pm 0.09$ & 0.0627 & 0.012 \\
control & 13 & $10.70 \pm 0.78$ & $1.38 \pm 0.30$ & & 0.029 \\
see figure 4.5 and 4.7 & & & & & \\
\hline $\begin{array}{l}5 \mu M \text { OA } \\
\text { control }\end{array}$ & 9 & $12.31 \pm 0.84$ & $2.81 \pm 0.46$ & \multirow{2}{*}{0.0020} & 0.051 \\
see figure 4.8 & 11 & $13.43 \pm 1.17$ & $1.40 \pm 0.27$ & & 0.023 \\
\hline
\end{tabular}

Table 4.1: For each pharmacological treatment (column one), there were also measurements performed on control boutons. Column two lists the number of experiments (n) performed by Jordan (2000). Column three and four list the mean and variance after reanalysis of the data. Column five lists the p-value for the variance over mean ratio between control and treated boutons (unpaired t-test with unknown variance). Column six lists the $\frac{N_{m}}{N_{t}}$ ratio calculated according to equation 4.2 .

when the broadband phosphatase blocker okadaic acid was applied. In the following sections, the obtained data for control cells and these two treatments will be analyzed using Monte-Carlo simulations. Table 4.1 shows the results of the variance analysis for these two treatments and the corresponding control experiments.

The mean of a fluorescence trace is a measure for the number of the vesicles in the detection volume, and the variance (after shot-noise correction) a measure for their mobility. Changes in the variance over mean ratio indicate a change in vesicle mobility. However, power spectra or autocorrelation analysis provide additional information on how the mobility changed (in the frequency or time domain respectively). The FFS experiments thus delivered two crucial parameters (i.e. the variance over mean ratio, and the PS or ACF) that were dependent on the type of movement the objects undergo in the detection volume.

As explained above, the hitherto described analytical models were not sufficient to describe the data obtained in the FFS experiments, so that Monte-Carlo simulations of vesicle mobility were carried out (see section 3.1 for details). Most parameters of the simulation were known from control experiments and could be fixed in the simulation. These are:

1. The shape of the confocal detection volume (known from calibration experiments, see Jordan (2000) for details)

2. The shape of the synapse (approximately a sphere, $\varnothing=1 \mu \mathrm{m}$, Schikorski and Stevens (1997, 2001)) 
Figure 4.4: ACF of control data from ML-7 experiments (see table 4.1, and figure 4.5) in black. The blue ACF is a simulation with $D=$ $5 \cdot 10^{-5} \frac{\mu m^{2}}{s}$ and cage $e_{\text {radius }}=50 \mathrm{~nm}$ $\left(\right.$ mean $_{\text {sim }}=13.14 \mathrm{kcps}, \mathrm{var}_{\text {sim }}=$ $\left.1.28 \mathrm{kcps}^{2}\right)$.

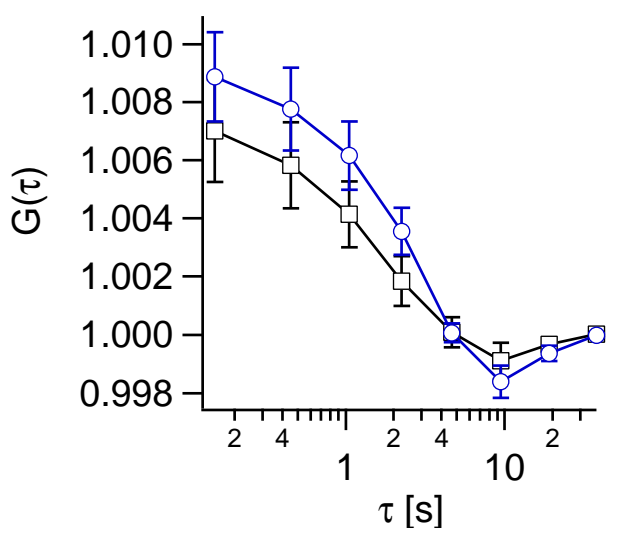

3. The brightness of a single vesicle (cpv, see figure 4.3)

Knowing that the synapse is a highly specialized and fine-tuned machinery to perform the task of vesicle endo-exocytosis, one can suggest the model that vesicles are anchored at specific locations by certain proteins. This model was tested first by simulating that vesicles can move only within a small region, the cage, with diffusion coefficients $D$ similar to those found in other synaptic or model systems (Holt et al., 2004; Rea et al., 2004; Steyer et al., 1997; Zenisek et al., 2000). The value of $D$ was varied in the simulations with $D=5 \cdot 10^{-3}-5 \cdot 10^{-6} \frac{\mu m^{2}}{s}$ and for the cage, cage $_{\text {radius }}=12.5-200 \mathrm{~nm}$. Since we had two readout parameters to characterize vesicle mobility (the mean over variance ratio and the PS or ACF analysis), and as the simulations had only two parameters (the cage size and D), explicit results for these parameters were expected. Figure 4.4 shows that the simulation was capable of approximating the shape of the measured autocorrelation function. Additionally, the slope of the variance over mean was similar to the experimental data (see figure 4.1).

Autocorrelation and power spectral analysis are mathematically related to each other by the Fourier transform. While the autocorrelation analysis includes phase information, this parameter is lost in the power spectral analysis. However, power spectra (PS) are a convenient tool to easily investigate changes in the frequency domain. As one can see in figure 4.1, the variance over mean ratio was quite variable, limiting the accuracy of this parameter and thus the reproducibility of the autocorrelation function (ACF) amplitude. Since no analytical model was available to interpret the ACF, the shape of the PS was inspected, which was very reproducible (e.g. see figure 4.5). For this reason, power spectral analysis together with a separate analysis of the variance over mean ratio were preferred in this thesis.

Jordan (2000) tested the effect of a variety of pharmacological treatments targeting 

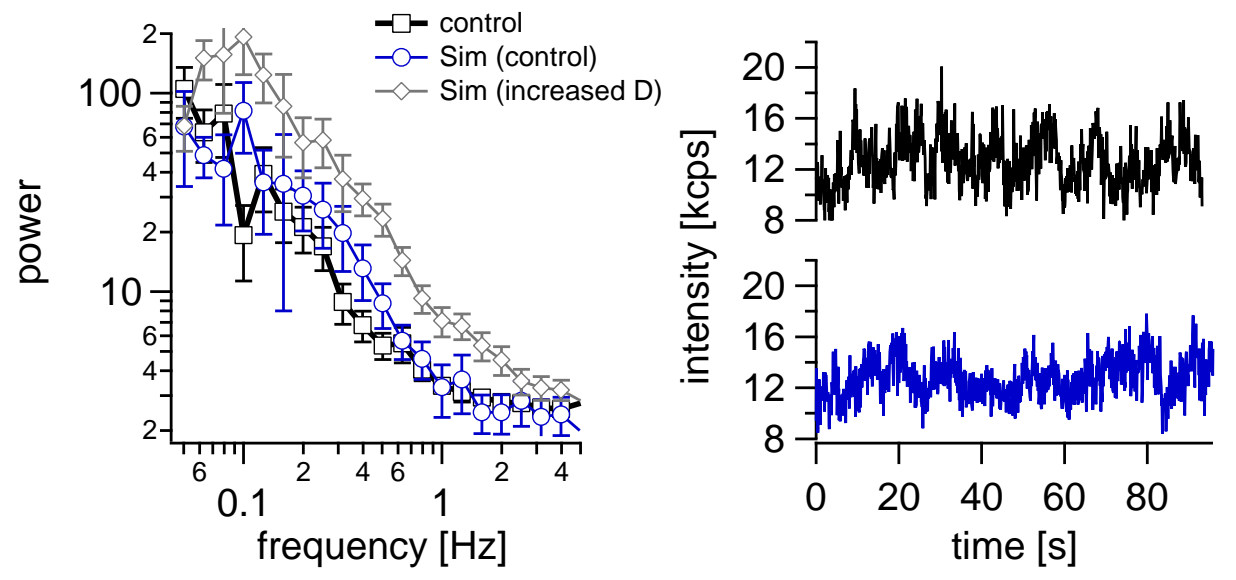

Figure 4.5: Power spectra of resting untreated synapses and simulated data. The left panel shows the power spectra of control cells from ML-7 experiments (see table 4.1) in black, as well as a simulation of this data with $D=5 \cdot 10^{-5} \frac{\mu m^{2}}{\mathrm{~s}}$ and $r_{\text {cage }}=50 \mathrm{~nm}$ $\left(\right.$ mean $\left._{\text {sim }}=10.8 \mathrm{kcps}, \mathrm{var}_{\text {sim }}=1.1 \mathrm{kcps}^{2}\right)$. The grey power spectrum is a simulation with a twofold higher D but same cage size. Its shape differs from the one in blue and the variance also increased $\left(\right.$ mean $\left._{\text {sim }}=13.7 k c p s, v r_{s i m}=2.77 k c p s^{2}\right)$. The right panel shows individual fluorescence traces of control cells (black) and simulation (blue).

kinases, phosphotases and cytoskeletal elements. To propose a quantitative model of vesicle mobility, some of this data is summarized in the following paragraphs together with results from simulations.

\section{Accuracy of simulated parameters}

Simulation were carried out spanning a 10000 fold range for D. The cage radius was varied ranging from 12.5 to $200 \mathrm{~nm}$ (see appendix $\mathrm{B}$ ). For $D=5 \cdot 10^{-5} \frac{\mu m^{2}}{\mathrm{~s}}$ and a cage radius of $50 \mathrm{~nm}$, doubling $\mathrm{D}$ resulted in a detectable shape change of the power spectrum (see figure 4.5). However, varying the cage radius (and fixing $D$ to $5 \cdot 10^{-5} \frac{\mu^{2}}{\mathrm{~s}}$ ) did not result in any obvious changes of the power spectra, and only in small changes of the variance (see also appendix $\mathrm{B}$ ). This is due to the high pass filtering at $0.05 \mathrm{~Hz}$ that had to be implemented to get reproducible measurements (Jordan, 2000). This filter removed all contributions to the variances from slow frequency components, which increased with growing cage size at a given D (see also appendix B). Figure 4.6 illustrates this effect, and the precision of our cage estimate was thus limited to approximately a factor of two. 
Figure 4.6: Effect of high pass filtering on the variance. The cage $_{\text {radius }}$ was varied with $D$ fixed to $5 \cdot 10^{-5} \frac{\mu m^{2}}{s}$. Plotted in circles is the variance for the unfiltered simulated data, and in squares for the $0.05 \mathrm{~Hz}$ high pass filtered data.

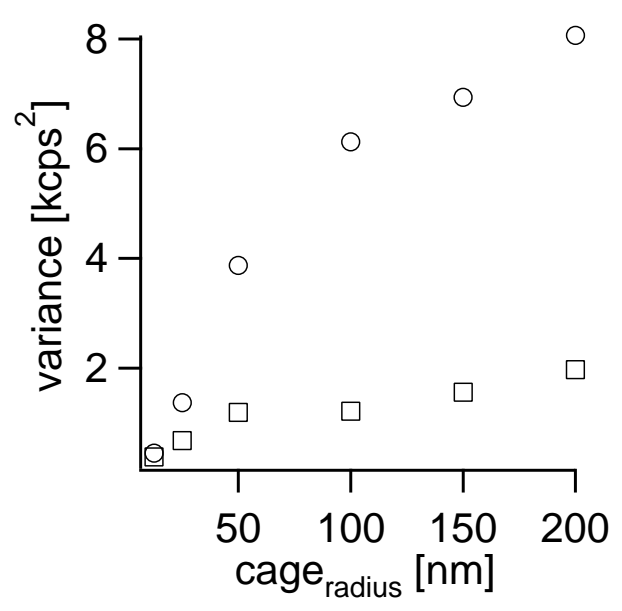

\section{Blockage of myosin light chain kinase}

The potent blocker of myosin light chain kinase (MLCK) ML-7, was proposed to have an effect on vesicle mobility by disabling the function of the motor protein myosin. However, this was only indirectly concluded from analyzing the staining and destaining properties of FM 1-43 stained synapses after blocking MLCK (Ryan, 1999).

FFS directly showed the impact of ML-7 on vesicle dynamics (Jordan, 2000). The power spectra showed a decrease in the amplitude at lower frequencies (figure $4.7)$, and the mean over variance ratio decreased $(\mathrm{p}=0.0627)$ by approximately twofold (see table 4.1). This decrease in variance was already visible if one compared the fluctuation time traces between wild type and control (compare figure 4.5 with 4.7). The data could be nicely simulated with a tenfold lower $\mathrm{D}$ than control conditions and similar cage radius $\left(D=5 \cdot 10^{-6} \frac{\mu \mathrm{m}^{2}}{\mathrm{~s}}\right.$ and $r_{\text {cage }}=50 \mathrm{~nm}$, mean $_{\text {sim }}=12.4 \mathrm{kcps}, \mathrm{var}_{\text {sim }}=0.18 \mathrm{kcps}^{2}$ ). Disabling motor proteins thus directly had an effect on vesicle mobility. Other possible side effects of ML-7 on PKA or PKC (Saitoh et al., 1987) were tested in control experiments in which these phophatases where specifically blocked. No effects on variance or power spectral analysis were detected by Jordan (2000).

\section{Treatment with okadaic acid}

Jordan (2000) specifically tested the involvement of cytoskeletal elements by targeting drugs to disrupt actin and tubulins. It first seemed a bit surprising that no effect was observed (Jordan, 2000). However, these experiments also served as a crucial control since there might have been some remaining concern that bouton (synapse) movement had a contribution to the observed variance. Since application of cytochalasin and 

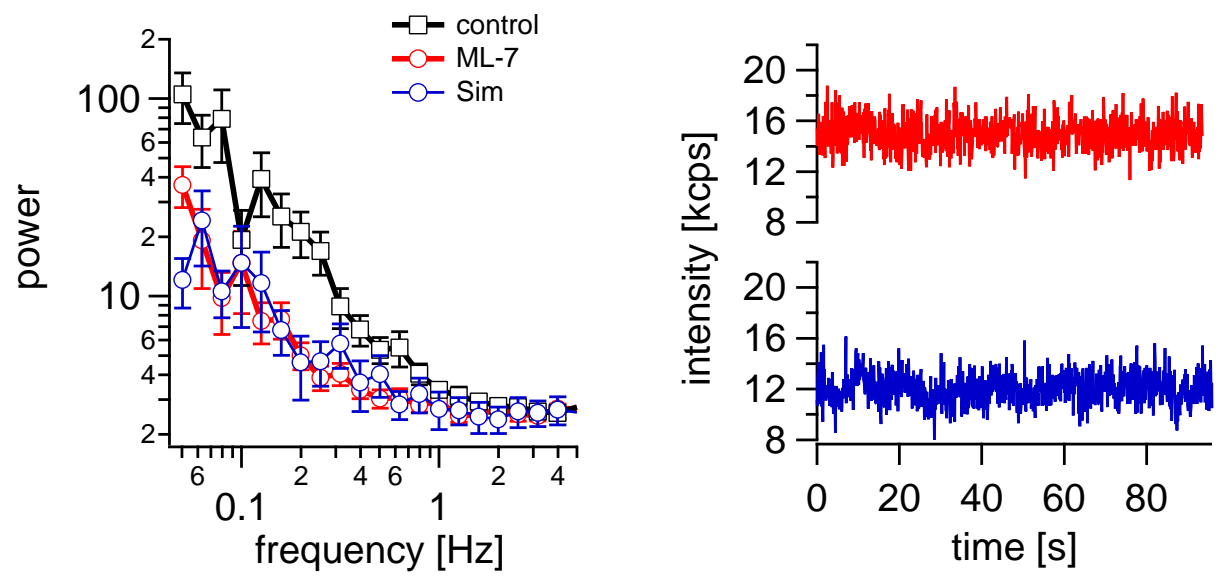

Figure 4.7: Power spectra and simulation of ML-7 treatment. The left panel shows the power spectra for ML-7 treatment in red, and the control experiment as a reference in black (control cells from ML-7 experiments, see table 4.1). A simulation with $D=$ $5 \cdot 10^{-6} \frac{\mu m^{2}}{s}$ and $r_{\text {cage }}=50 \mathrm{~nm}\left(\right.$ mean $_{\text {sim }}=12.4 \mathrm{kcps}$, var $\left._{\text {sim }}=0.18 \mathrm{kcps}^{2}\right)$ is overlaid in blue. The right panel exemplarily shows fluctuation time traces of ML-7 treated synapses (red) and the simulation (blue).

latrunculin (which promote actin depolymerization) were known to block synapse movement completely (Fischer et al., 1998), and no changes were observed in the mobility analysis, it could be ruled out that bouton movement had a significant effect on the outcome of the performed analysis. However, since myosins require actin as a guiding track in order to build a functional transport unit, the finding that actin disruption had no effect on the mobility seemed at odds with the finding that motor proteins were important (see preceding section). This suggested that another protein also anchors the vesicles in place, so that actin depolymerization in itself was not enough to increase mobility. Good candidate proteins were the synapsins (for a review see Greengard et al. (1993)). Jordan (2000) tested this hypothesis by applying the broadband phosphatase blocker okadaic acid (OA), which was known to disperse the synaptic vesicle cluster (Henkel et al., 1996, Kraszewski et al., 1995). Application of OA to hippocampal synapses led to increases in the high frequency range of the PS and to a significant increase in the variance to mean ratio (see figure 4.8 and table 4.1). Modelling this effect turned out to be more difficult. Good results were obtained by simulating vesicles with a 8 fold higher D than control cells, and even better if a vesicle mixture was assumed with only $25 \%$ of the vesicles having a hundred fold higher D than in the control simulations. Jordan $(2000)$ could also show that the OA effect was more pronounced with increasing concentrations of latrunculin B suggesting that vesicles were anchored to both synapsins and actin (indirectly via 

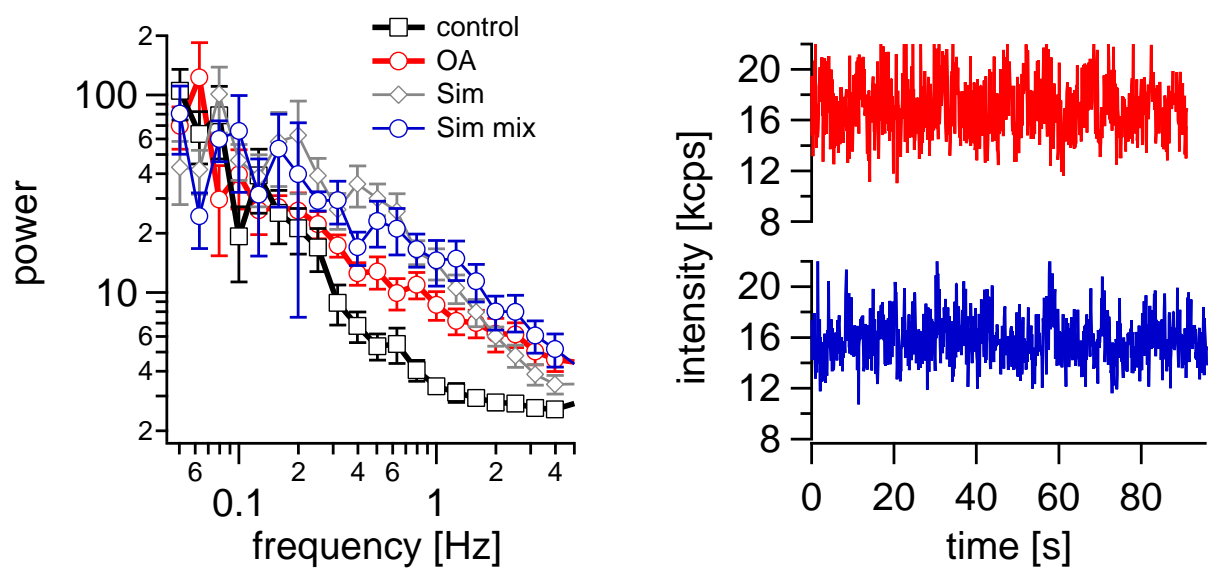

Figure 4.8: Power spectra and simulation of okadiac acid treatment. The left panel shows in red the power spectra of the experimental data from Jordan $(2000)$ after OA treatment. Additionally, two simulations are shown. A simulation with $D=4 \cdot 10^{-4} \frac{\mu^{2}}{\mathrm{~s}}$ and $r_{\text {cage }}=25 \mathrm{~nm}\left(\right.$ mean $_{\text {sim }}=11.98 \mathrm{kcps}$, var $\left._{\text {sim }}=1.75 \mathrm{kcps}^{2}\right)$ in grey rhombi and when $25 \%$ vesicles were simulated with $D=5 \cdot 10^{-3} \frac{\mu m^{2}}{s}, r_{\text {cage }}=50 \mathrm{~nm}$ and $75 \%$ with control conditions $D=5 \cdot 10^{-5} \frac{\mu m^{2}}{s}, r_{\text {cage }}=50 \mathrm{~nm}$ in blue circles $\left(\right.$ mean $_{\text {sim }}=$ $11.9 k c p s, v r_{\text {sim }}=2.44 \mathrm{kcps}^{2}$ ). The right panel shows fluctuation time traces of simulations with mixed vesicle population in blue, and those of okadaic acid-treated synapses in red.

myosin).

\subsection{Optimizing a setup for single vesicle tracking}

The aim of the following section is to describe the development of a setup that is ideally suited to monitor mobility of single fluorescently labelled vesicles in hippocampal synapses in real-time.

Fluorescent labelling of individual small synaptic vesicles can be achieved with the FM 1-43 styryl dyes using the two-step one-color staining protocol as outlined in figure 2.5 and already used in the preceding section. However, in a primary hippocampal culture, FM 1-43 can also unspecifically bind to the neuronal membrane, underlying astrocytes and dead cell material, as well as being constitutively endocytosed by cells. This non-synaptic fluorescence is not obvious if one works with fully stained and thus bright synapses, however, it is a major problem when trying to image single vesicles in real-time, since these images suffer from a low contrast. For example, Aravanis et al. (2003b) stated in their method section that they selected about 20 coverslips 
with growing neurons out of 600 to finally image approximately 40 individual vesicles in real-time with a wide field fluorescent microscope.

In this thesis, in order to decrease the influence of background staining and autofluorescence when imaging single vesicle fluorescence, confocal or two-photon microscopy were used. In the following paragraphs, preliminary results that were acquired using two-photon microscopy are reported, which led to the subsequent development of a dual-color single-photon confocal microscopy configuration.

\subsubsection{Imaging single synaptic vesicles using two-photon microscopy}

For real-time imaging of single vesicles using two-photon microscopy, the culture was stained using the two-step one-color protocol (see methods section 2.3 for details). In this protocol, the culture was first stained minimally, and a time series was acquired to monitor single vesicle. The existence of functional synapses in the regions of interest (ROIs) was then ascertained by another round of staining, image acquisition and destaining using strong stimulation of the synapse.

Single synaptic vesicles were stained minimally by application of 5 APs at $5 \mathrm{~Hz}$ during 10 s presence of FM 1-43 dye, similar to the experiments performed in section 4.1.1. The intensity of individual fluorescent spots was measured by integrating over all pixels in a region of interest $(\varnothing=17$ pixel) and plotted in a histogram. The obtained intensity histogram of individual fluorescent spots showed the expected quantal distribution with three peaks (see figure 4.9 and methods section 2.3 for details on obtaining and analyzing these histograms), corresponding to synapses with one, two, or three stained vesicles, respectively. Those fluorescent spots with intensities belonging to the first peak -corresponding to synapses with a single stained vesiclewere further analyzed in the time series (off-line). In every frame, the position of a fluorescent spot originating from a single vesicle was determined using a fit with a 2D Gaussian. To analyze the mobility of vesicles, the root mean square displacement of vesicle positions in the time series was calculated $\left(R M S=\sqrt{\left(\frac{1}{N} \cdot \sum y_{i}\right)}\right), \mathrm{N}=$ number of points, and $y_{i}$ the displacement from the first image). The RMS was an average measure of how far from the initial origin the object moved. Figure 4.10 summarizes the RMS obtained for 47 single vesicles. The observed mobility may to some degree reflect the error in the precision of the position determination. To estimate the limits of this precision, synapses were minimally stained and fixed with 
Figure 4.9: Histogram of green fluorescent puncta. The intensity of a putative single vesicle was determined by measuring the number of photons after background correction in a circular region of $17 \mathrm{pxl}$. The histogram shows the quantal distribution of measured intensities, and was fitted with a sum of three Gaussian according to equation 2.1. 162 regions of interest were analyzed. The peaks were centered at integer numbers of $\mu=191$ photons. The first peak corresponds to boutons stained with only one vesicle, the second peak to those with two stained vesicles, etc..

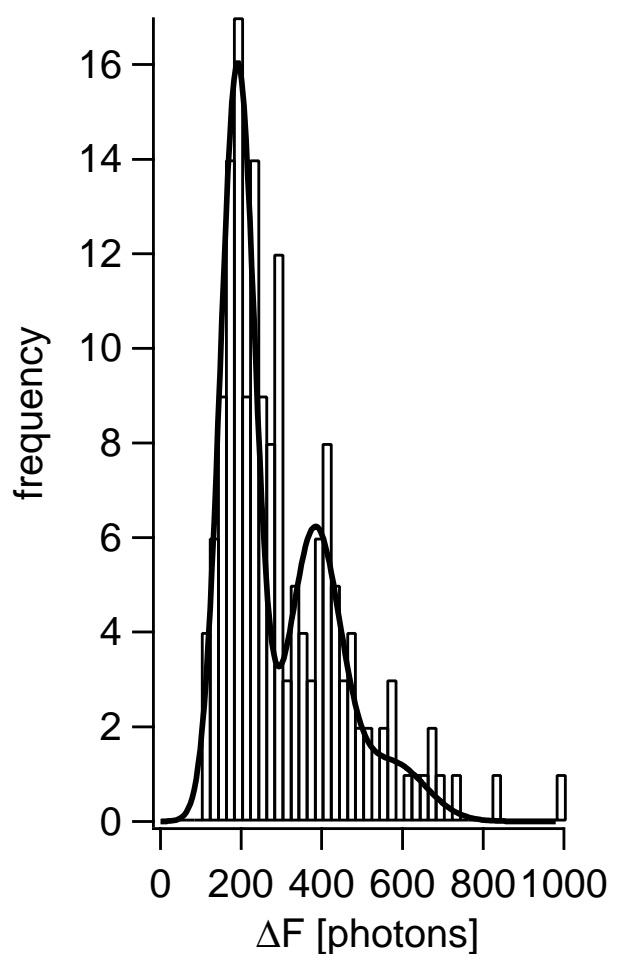

$4 \%$ paraformaldehyde, after which a time series was acquired. The RMS of spots having a similar intensity as the single vesicles in the unfixed culture was analyzed from this time series. The obtained RMS for these spots is also depicted in figure 4.10. Please note that the RMS of fluorescent spots in the fixed cultures is a measure of the precision limit of the tracking experiment. The mobility of vesicles in living cultures was thus higher than the limit of the tracking precision. Even though RMS analysis was a very crude analysis on vesicle mobility, several points could be learned from the performed experiment:

Figure 4.10: RMS analysis of single vesicles. 47 single vesicles belonging to the first peak in figure 4.9 were tracked for at least 20 frames (pixel dwell time $1 \mu \mathrm{s})$. As a crude analysis on overall vesicle mobility, the root mean square displacement was analyzed (shown in red). The RMS of 33 putative vesicles from a culture fixed with $4 \%$ paraformaldehyde is shown in blue.

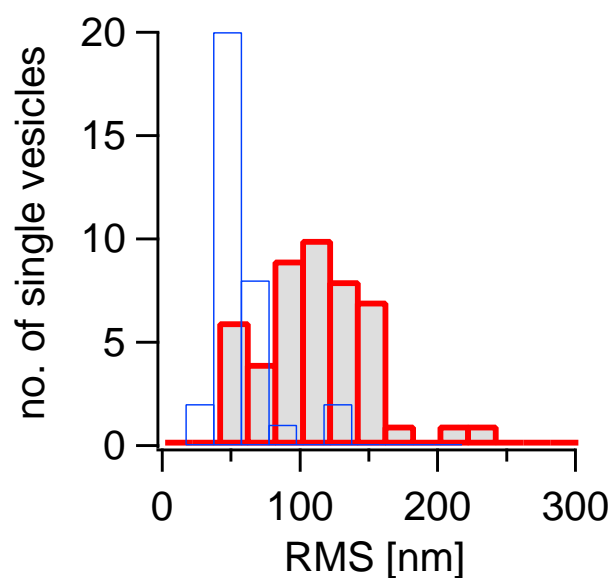


1. Finding the focus and a proper imaging region in a culture only minimally stained was very difficult due to the low contrast of such images. One had to be very careful that no prebleaching occurred while adjusting the focus.

2. After each tracking experiment, the culture had to be stained again to identify fully functional synapses. If any form of image drift or cell movement happened during this time, the coverslip usually had to be excluded from analysis.

3. The RMS plot in figure 4.10 indicated that some vesicles were also quite mobile $($ RMS $>150 \mathrm{~nm})$. One of the biggest and most important concerns was: Did the vesicle or possibly the whole synapse move? Slow synapse mobility is a known phenomena in neurobiology (Fischer et al., 1998). It seemed impossible to eliminate this concern if only individual vesicles were labelled. Unfortunately, hippocampal synapses were very often too small $(\varnothing \sim 1 \mu \mathrm{m})$ or had too low contrast to be identified properly in a Nomarski image. It was thus not possible to determine if a synapse moved by conventional light microscopy techniques such as phase contrast imaging.

4. A strong bleaching of dye by the two-photon excitation was observed (see appendix C for details).

All these described problems led to the development of a new dual-color setup, in which simultaneous imaging of the vesicles and the synapses was possible, using the green fluorescent styryl dye FM 1-43 and the red fluorescent dye FM 5-95. The characterization of this dual-color single photon excitation setup is outlined in detail in appendix C. The most important changes were the use of a single-photon laser as opposed to the previous two-photon excitation, which reduced bleaching, and the use of a custom-made four APD detector unit, which increased the sensitivity and linearity of the detection system.

The dual-color single-photon setup was superior to the two-photon setup in all regards and was used in all following vesicle tracking experiments. Single vesicles and synapses were tracked using a Gaussian fit and a crosscorrelation algorithm respectively (see section 3.3 for details). If a small shift (see also section 3.3 for details) of the synapse was detected, the synapse and the corresponding vesicle were removed from analysis. Thus, now one could be sure that any movement detected in the green fluorescent channel could only originate from vesicle movement. 
In addition to the technical advantages of the dual-color setup, there were also some practical assets. As outlined in methods section 2.3, in the dual-color experiments a two-step staining protocol was used to first stain the complete recycling pool of the synapses with a red fluorescent dye before staining some vesicles in green with a minimal stimulation protocol. Staining synapses first had a tremendous experimental advantage, since regions of proper imaging quality (e.g. nicely separated synapses and minimal background staining) could be preselected. This reduced the time spent collecting data on single vesicles. Furthermore, due to the bright red fluorescent spots, the optimal focus plane could be adjusted easily.

\subsection{Single synaptic vesicle tracking using dual color imaging}

In the following section, the dual-color protocol (recall figure 2.5) was employed on the single-photon setup (described in the preceding section and in figure 2.7) to monitor single vesicle mobility. Single vesicles were identified and tracked using the algorithm introduced in section 3.3. Dynamics of vesicles that were endocytosed via different modes will be analyzed during resting conditions and during stimulation of the synapses.

The first step in any vesicle tracking experiment was to solve the problem of labelling and identifying single vesicles which was performed according to the two-step dual-color protocol.

First, synapses were stained using the red fluorescent dye FM 5-95 and a strong stimulus. An additional staining protocol was then applied to only minimally stain the synapses, aiming to stain only one vesicle per synapse. In this section, four different protocols will be outlined to minimally stain individual synapses. Every condition will be analyzed in the same way using the algorithm described in section 3.3 , and the results for each condition will be listed in a separate paragraph and summarized at the end of this section.

The most important issue was to ensure that only single vesicles were tracked. As outlined in the methods section 3.3.2, this was done by various selection criteria, the intensity of the fluorescent object being the most important. 
The typical intensity of a single dye-stained synaptic vesicle was already determined in the results section 4.1.1, although under slightly different experimental conditions. While those experiments were performed on the same setup, they used the initial two-step single-color (rather than dual-color) protocol to identify single fluorescent vesicles. The laser was operated at half intensity, and a different emission filter was used, which allowed for collecting twice as much green fluorescent light as in the dual-color experiments. Taking into account these parameters, it was expected for the following dual-color experiments that a single vesicle should have an approximate intensity of 100 photons (for pixel dwell time please refer to section 2.3.2)

\subsubsection{Rapidly endocytosed vesicles}

In the work by Aravanis et al. (2003b), Ryan et al. (1997), and Murthy and Stevens (1999) single vesicles were stained by weak stimulation during a brief presence of FM 1-43 dye. This allowed only those vesicles that were endocytosed simultaneously with the arrival of the stimulus to be stained. These vesicles will be referred to as rapidly or fast endocytosed vesicles.

Aravanis et al. (2003b) investigated the exocytotic dynamics of these vesicles during stimulation using real-time imaging, and suggested that they are rapidly reused and capable of undergoing exocytosis as a kiss and run event. However, no particle tracking studies on the single vesicle level were possible so far, which was the aim of the work described in the following paragraphs.

\section{Single vesicle histogram of rapidly endocytosed vesicles}

To investigate the mobility of these rapidly endocytosed vesicles, it was absolutely necessary to restrict analysis to those fluorescent puncta originating from one stained vesicle per synapse, since particle tracking algorithms are not reliable if two fluorescent objects are in close vicinity. To achieve this aim, the minimal staining protocol was applied in the following way: FM 1-43 was applied for $10 \mathrm{~s}$ to the medium during stimulation with 3 APs at $5 \mathrm{~Hz}$ (starting one second after dye application). Regions of interest (ROIs) were those regions where in the red channel a bright fluorescent object was visible, and in the green fluorescent channel a fluorescent spot. In both channels, the fluorescence had to be destainable in the course of further stimulation of the culture (for details on selecting ROIs refer to section 3.3.2). The $\Delta F$ intensities of the green fluorescent spots were plotted (measured in a box region with edge length $=11$ 
pixel before and after destaining of the synapse) in a histogram yielding the expected peaked quantal distribution (see figure 4.11). Compared to the similar histogram analysis in the preceding section (section 4.1.1 and 4.2.1), this histogram could not be fitted satisfactorily with the model proposed by Murthy and Stevens (1998) (equation 2.1 with $\mu_{\text {offset }}=0$ ), because the histogram showed a small intensity offset. This offset originated from background fluorescence in the green fluorescent channel that was present everywhere in the green channel where a synapse was identified after staining of the culture with the red fluorescent dye (even in the absence of any FM 1-43). The reason for this offset was a minor bleed-through of the red fluorescent light into the green fluorescent detection channel. The background fluorescence was determined by measuring regions centered on red synapses that did not show any obvious staining in the green channel ( $<60$ photons, see figure 4.11 , and please refer to section 3.3.2, where details are given on how those regions were selected). The result showed a Gaussian distribution centered around 13 photons, which was the value used for $\mu_{\text {offset }}$ in equation 2.1, which now yielded a good fit of the experimental data in the histogram (see figure 4.11).

\section{Fusion of rapidly endocytosed single vesicles}

For the following sections, it will be useful to recall the stimulation protocol that was applied during acquisition of the time series (see section 2.3.2). For the first 15 frames $(1$ frame $=1.512 \mathrm{~s}, 15$ frames $=22.68 \mathrm{~s})$, the culture was at rest (indicated with blue bars). The culture was then stimulated at $5 \mathrm{~Hz}$ for another 15 frames (frames 16-30 $=22.68 \mathrm{~s}$, indicated with red bars) and then starting from the beginning of frame 31 with $20 \mathrm{~Hz}$ (black bars) until the end of the time series acquisition.

As explained in the methods section 3.3.2, a fusion event was determined as a drop of $>40 \%$ in the fluorescence amplitude (determined by a Gaussian fit to the fluorescent spot) of the single vesicle due to stimulation. 22 single vesicles were taken for analysis (see section 3.3 .2 for selection criteria). Out of these vesicles, $68 \%$ fused during the first 75 APs (frames 16-30) of the weak $5 \mathrm{~Hz}$ stimulus. This is in agreement with the data from Aravanis et al. (2003b), who also found that rapidly endocytosed vesicles had a very high release probability during another round of action potential stimulus.

Figure 4.12 shows a histogram of the distribution of these fusion events over time, while in the left panel of figure 4.13 the absolute fluorescence intensity (not the amplitude of the Gaussian fit) as a function of time of these 22 vesicles are shown. 

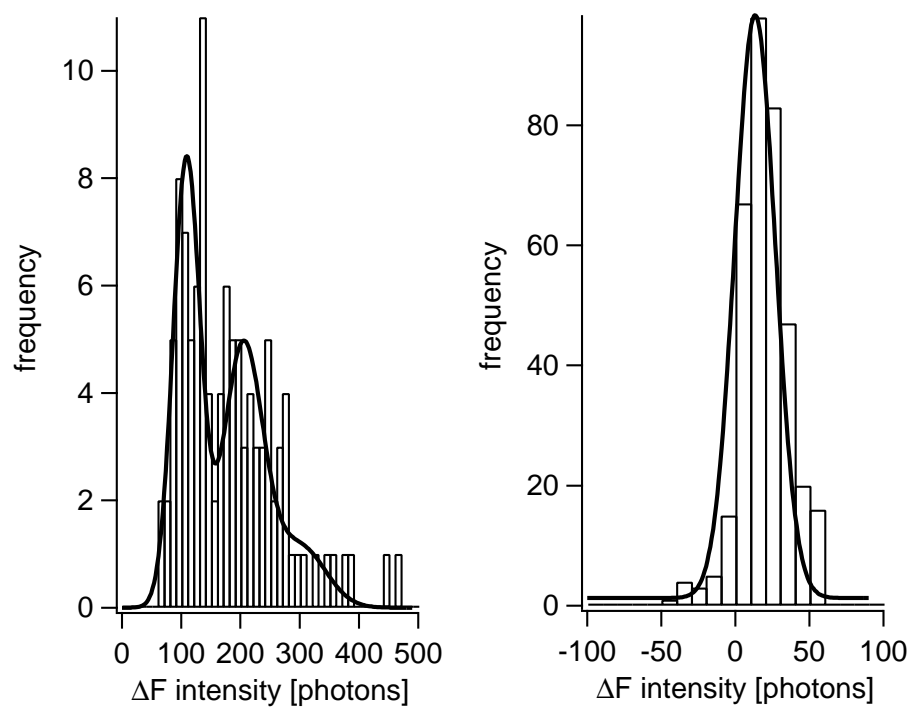

Figure 4.11: Rapidly endocytosed vesicle histogram. The left panel shows the quantal intensity histogram $(\mathrm{N}=109)$ that was fitted (black solid line) using equation 2.1 yielding $\mu=91$ photons. The width of the first Gaussian was $\sigma=22$ photons, and only vesicles within $95 \%$ confidence of the the first peak were taken for analysis. The right panel shows the distribution of the background intensity in ROIs (in the green channel) where only red synaptic staining was apparent in the red channel but no green fluorescent puncta in the green channel $(\mathrm{N}=331)$. This bleed-through effect caused the offset in the left histogram. A fit with a single Gaussian returned $\mu_{\text {of } f s e t}=13$ photons. This parameter was used for fitting the single vesicle histogram in the left panel.

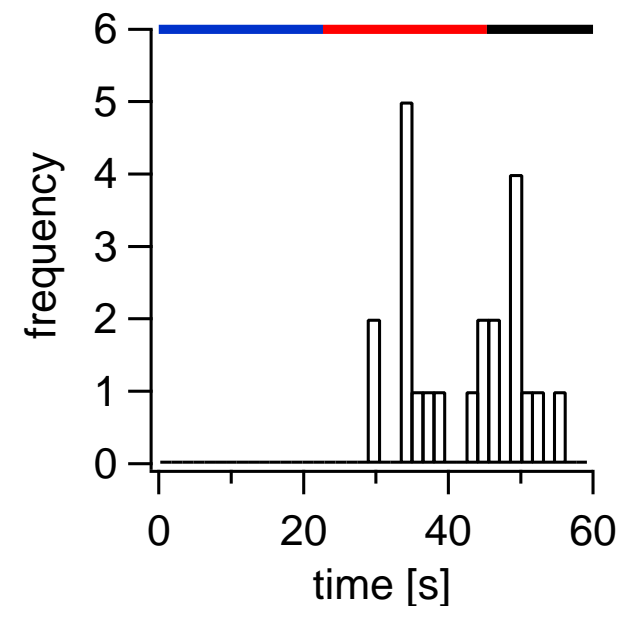

Figure 4.12: This histogram shows the distribution of fusion events of the 22 single vesicles as a function of time. Blue bar $=$ no stimulus, red $=$ $5 \mathrm{~Hz}$ stimulus and black $20 \mathrm{~Hz}$ stimulus. The stimulus protocol started 10 min after minimal staining of the culture (see figure 2.5 for details). 

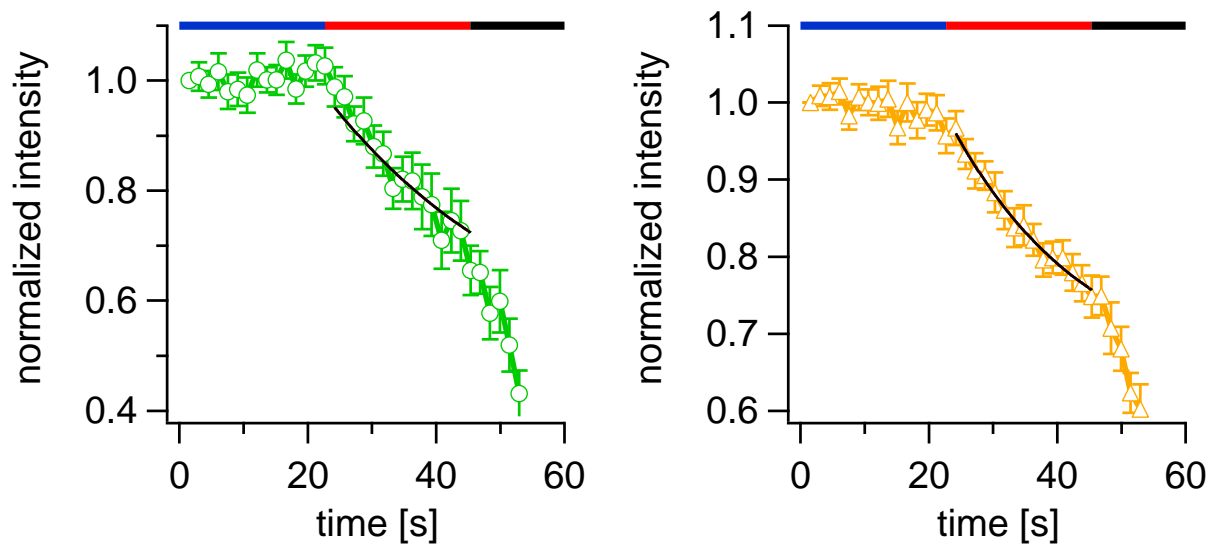

Figure 4.13: Plot of the normalized average intensity of the vesicles (left panel) and the corresponding synapses (right panel) versus time. A mono-exponential fit to the averaged single vesicle fluorescence destaining during mild stimulation (75 APs in total for frame 16 to 30 which is equal to $\sim 22.7 \mathrm{~s}$ ) yielded $\tau_{\text {ves }}=33.2 \mathrm{~s}$ and for the synapses that were stained in red using a strong stimulation protocol with $\tau_{\text {synapse }}=21.5 \mathrm{~s}$.

To allow for later comparison of different experimental conditions (see summary in paragraph 4.3.5 it was important to know whether the cultures were always stimulated in a similar way throughout all experiments. For this reason, an additional analysis of the synapse intensity over time was performed. The right panel in figure 4.13 summarizes the obtained destaining kinetics of synapses.

Please note that avalanche photo diodes were used as detectors in this study. These display a non-linear detection behavior, especially at high photon fluxes. All intensity measurements in the time series recordings (where the pixel dwell time was short, see section 2.3 .2 for details on image acquisition) can thus be biased due to the nonlinearity of the detectors. This is especially a concern in the red channel, where synapses appeared as bright fluorescent objects. All absolute values from analysis of destaining kinetics should thus be taken with some caution.

\section{Mobility of rapidly endocytosed single vesicles}

For those single vesicles passing all the selection criteria outlined in section 3.3.2, the positions were determined in every frame up to two frames before the detected release event. Please remember that the last two frames before the detected release event were discarded to rule out the possibility that dye that was just released but not completely washed away, which could lower the precision of the tracking algorithm. 


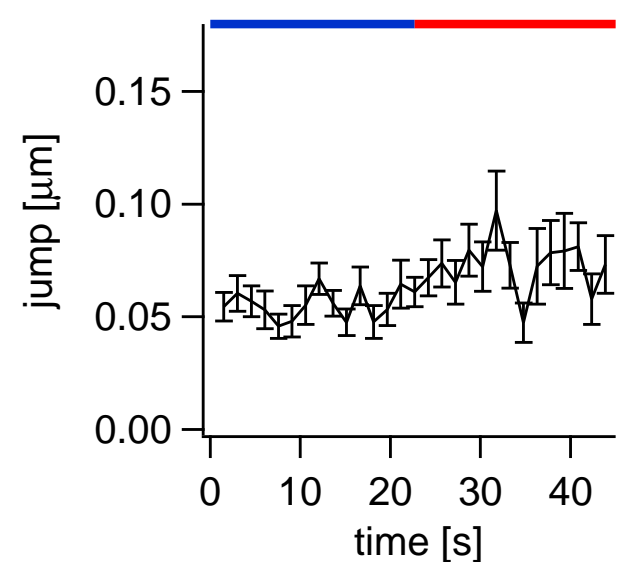

Figure 4.14: Frame-to-frame displacement, i.e. the distance how far a single vesicle moves from one frame to the next (jump) is plotted versus the time $(1$ frame $=1.512 \mathrm{~s})$. Shown is the average over the 22 vesicles. The error bars are in s.e.m..

Furthermore, all of the following mobility analysis was only carried out until frame 30 , which is the end of the mild stimulus of $5 \mathrm{~Hz}$. Since vesicles fused after very few stimuli, not enough frames for a proper statistical analysis for vesicle movement during the high frequency stimulus were available.

To visualize the average mobility of single vesicles, the jump distances from one frame to the next were analyzed and plotted in figure 4.14 (this type of analysis will be referred to as the overall mobility analysis). The average jump seemed to be very small $(<100 \mathrm{~nm})$ and seemed to increase slightly during stimulation. Please note, that, due to fusion, the number of vesicles analyzed decreases with time. In the following paragraphs more powerful analysis techniques were used to investigate this tendency.

\section{Comparing mobility of vesicles at rest and during stimulation}

The following paragraphs aim to analyze if stimulation indeed caused an increase in vesicle movement. For this purpose, the movement of vesicles during stimulation is compared to the best available control, i.e. the same vesicle during resting conditions. The following analysis is best described using two examples:

Example 1 A fusion event was detected in frame 34. Frames 1-15 (15 frames in total) of this vesicle where the culture was at rest were analyzed and compared to the analysis of frame 16-30 (also 15 frames in total) where the culture was stimulated continuously at $5 \mathrm{~Hz}$.

Example 2 A fusion event was detected in frame 29. The analysis is thus restricted to frames up to and including frame 27. For the resting conditions, frames 4-15 are 
analyzed, and for the stimulation conditions frames 16-27.

Please note that to allow for a proper statistical comparison, always the same number of frames are analyzed for both conditions.

This type of analysis will be used in all following paragraphs dealing with mean square displacement (MSD) and jump frequency analysis (see below).

\section{MSD analysis of rapidly endocytosed single vesicles}

The mean square displacement is a powerful analysis technique for studying single

object dynamics (Saxton, 1993) and was previously used to study e.g. granule dynamics in endocrine cells (Steyer et al., 1997). The MSD plotted versus time carries information about the type of movement taking place. In the case of free diffusion, such a plot depicts a line and the diffusion coefficient can be calculated from the slope using the Einstein-Smoluchowski relationship,

$$
D_{2 D}=\lim _{t \rightarrow \infty} \frac{1}{4 t}\left\langle r^{2}\right\rangle
$$

with time $t, D_{2 D}=$ the two dimensional diffusion coefficient and $\left\langle r^{2}\right\rangle$, the average of the square of the mean displacement. In figure 4.15 mean square displacement analysis was performed on the same vesicles and number of frames before and during weak stimulation. Please note that it is inherent to MSD analysis that the points of highest statistical significance are the first few points, and in addition, due to fusion of vesicles the number of vesicles analyzed also decreased with time. However, the plot for the unstimulated condition seemed to saturate, which was an indication for confined diffusion. The MSD for the stimulated culture was shifted upwards, which indicated mobilization. An analytical interpretation of the MSD with a confined diffusion model needs a multi-parameter fit, which only returns meaningful results if sufficient data points are available. Due to the low time resolution in the experiments, the first points in the MSD that should show a decline towards zero are missing. Furthermore, due to the limited precision of the tracking algorithm, even at very high time resolution the MSD would show a zero offset. In order to avoid the danger of over-interpreting the data, the conclusion from the MSD was only a qualitative one, namely that the vesicle movement was confined and that mobility seemed to increase during stimulation. 


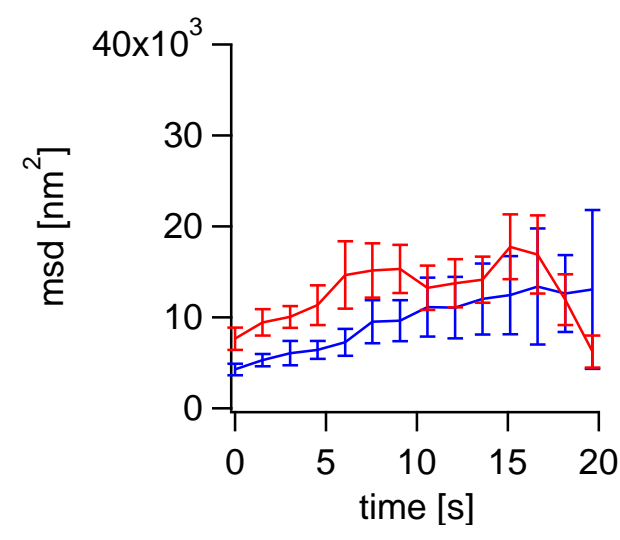

Figure 4.15: MSD analysis for rapidly endocytosed vesicles $(\mathrm{N}=22)$. Analysis of the vesicles during resting conditions is shown in blue and during stimulation in red.

\section{Jump frequency distribution analysis of rapidly endocytosed vesicles}

Fortunately, there is another type of analysis for studying single object dynamics called the jump frequency distribution (Crank, 1975). Analytically, it is very similar to the MSD analysis, however, the biggest advantage is that the data is not averaged and subpopulations can be identified. The method has been successfully used e.g. to identify three different mobility subpopulations of U1 splicing factors in the cell nucleus (Kues et al. 2001). In this analysis, the frequency of jump distances between $\Delta n$ frames is plotted in a histogram and can be analytically fitted with

$$
p(r, t) \Delta r=\sum_{j=1}^{k} \frac{M f_{i}}{2 D_{j} t} e^{\frac{-r^{2}}{4 D_{j} t}} r \Delta r,
$$

to yield $D_{j}=$ two dimensional diffusion coefficient for species $j$, with $t$ the lag time between $\Delta n$ frames, $M$ the number of jumps analyzed, $f_{j}$ the fraction of species $j$ with $\sum_{i=0}^{\infty} f_{j}=1$ and $r$ the jump distance and $\Delta r$ the analyzed jump interval (the bin size in the histogram plot, see below).

First, to get the best estimate of $D_{j}$ and $f_{j}$ for rapidly endocytosed vesicles under resting conditions, "all" frames acquired before stimulation of the 22 vesicles were plotted in a histogram (figure 4.16) and analyzed (all frames were the culture was at rest, $\mathrm{N}=308 ; 22$ vesicles times 14 for $\Delta n$ frames $=1$ ). The data could be fitted with a one-component model $\left(f_{j}=1\right)$ resulting in $D=5.12 \cdot 10^{-4} \frac{\mu m^{2}}{s}$. However, this fit ignored some data points for larger jumps. Alternatively the data was fitted with a two-component model giving $f_{1}=0.80, D_{1}=4.39 \cdot 10^{-4} \frac{\mu m^{2}}{\mathrm{~s}}$ and a faster component with $f_{2}=0.20, D_{1}=1.74 \cdot 10^{-3} \frac{\mu m^{2}}{s}$ (figure 4.16). The two-component fit seemed to describe the real data better, however this fit also had more parameters, and it is an inherent problem to data fitting that increasing the number of parameters will always 
Figure 4.16:

Jump frequency distribution analysis from rapidly endocytosed vesicles. Plotted are 308 frame to frame jump distances $(\Delta n$ frames $=1)$ over the jump distance $\mathrm{r}$ (bin size $\Delta \mathrm{r}=0.005$ $\mu m$ ). The data was fitted assuming one component of a freely diffusing species (green solid line). This fit however seems to miss data points for higher jump distances. An alternative fit assuming two components is also shown in black.

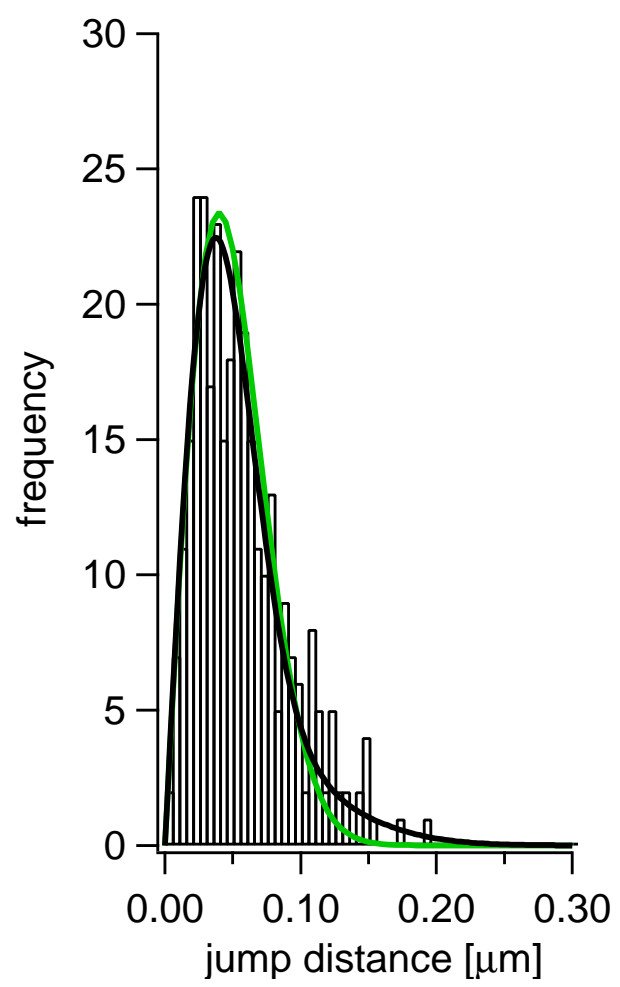

increase the quality of the fit.

Are there really two components? Statistics provides us with an almost unlimited repertoire of tools to analyze experimental data. For the problem of fitting the jump frequency distribution with one or more components, an excellent tool is the Akaike Information Criterion (AIC; Akaike (1981)). AIC allows to compare different types of fits to a model and to determine the best one. This is done by comparing how good a model is describing the data in terms of the maximum likelihood of this model and penalizing for the number of parameters used by the model, since increasing the number of parameters also increases the variance of the fit. Figure 4.17 shows that the AIC was better (smaller) using the two-component fit suggesting that there were indeed two populations of vesicle movement. A fit assuming more than two components did not even converge and could thus not be tested.

The bigger subpopulation $(80 \%)$ with $D_{1}=4.39 \cdot 10^{-4} \frac{\mu m^{2}}{s}$ represents an almost immobile fraction, since an average precision of the tracking algorithm can be estimated from the diffusion coefficient to be $\sigma_{\text {precision }}=\sqrt{\frac{D \cdot t}{4}} \sim 35 \mathrm{~nm}$ (compare to the precision obtained from simulations in section 3.3).

It was then interesting to investigate what happened to vesicle mobility during stim- 


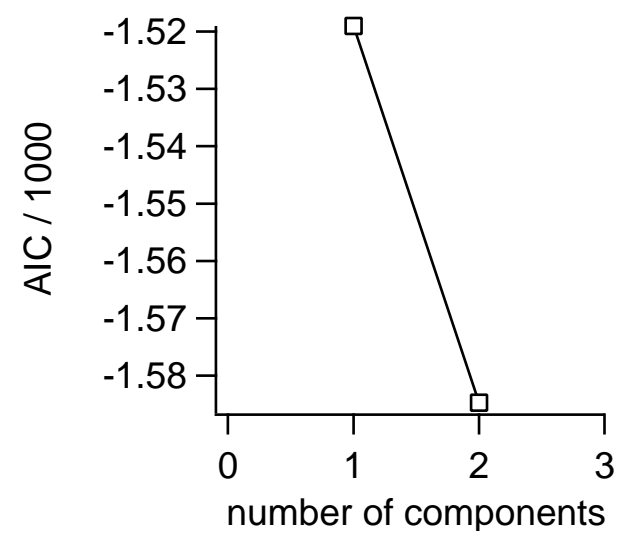

Figure 4.17: AIC plotted versus the number of components used by the fit according to equation 4.4 . The number of parameters for the one-component fit was 1 and for the two-component fit was three. Adding another two parameters and performing a three-component fit was not possible because the fit did not converge.

ulation of the culture. Figure 4.18 now shows the jump frequency histograms for the same number of jumps $(\mathrm{N}=194$, see the above given examples on how these frames were picked) analyzed before stimulation (in blue) and during weak stimulation (in red). In this case, the red histograms looks as if the blue one was "pushed down" from the top. The histograms were then fitted using a two-component model, where the diffusion coefficient of the immobile component was fixed. Not fixing the immobile component resulted in similarly good fits, but the variance in each fitted parameter made an easy comparison of different histograms (fits of histograms) more difficult. The fit for the blue histogram revealed $f_{1}^{\text {nostim }}=0.83, D_{1}^{\text {nostim }}=4.39 \cdot 10^{-4} \frac{\mu m^{2}}{\mathrm{~s}}(\mathrm{fixed})$ and $f_{2}^{\text {nostim }}=0.17, D_{2}^{\text {nostim }}=2.95 \cdot 10^{-3} \frac{\mu m^{2}}{\mathrm{~s}}$. This was almost identical to analyzing all jumps under resting conditions (see above). For vesicles during stimulation the fit resulted in an increase of the mobile fraction $f_{1}^{\text {stim }}=0.46, D_{1}^{\text {stim }}=4.39 \cdot 10^{-4} \frac{\mu m^{2}}{s}($ fixed $)$ and $f_{2}^{\text {stim }}=0.44, D_{2}^{\text {stim }}=1.75 \cdot 10^{-3} \frac{\mu m^{2}}{s}$.

Are the two distributions significantly different? The fit suggested that vesicles were mobilized due to stimulation of the culture. It was important to verify that the distribution of the stimulated vesicles was significantly different from the resting conditions. The Kolmogorov-Smirnov (K-S) test allows to test if two normalized cumulative distribution functions (cdf) are significantly different or not. A K-S analysis was performed for the two distribution functions. Figure 4.19 shows that the two jump frequency distributions were indeed significantly different. The KS-distance, here defined as the maximum length of a vertical line between the data and the diagonal of a $95 \%$ confidence bound, was 0.08 . 

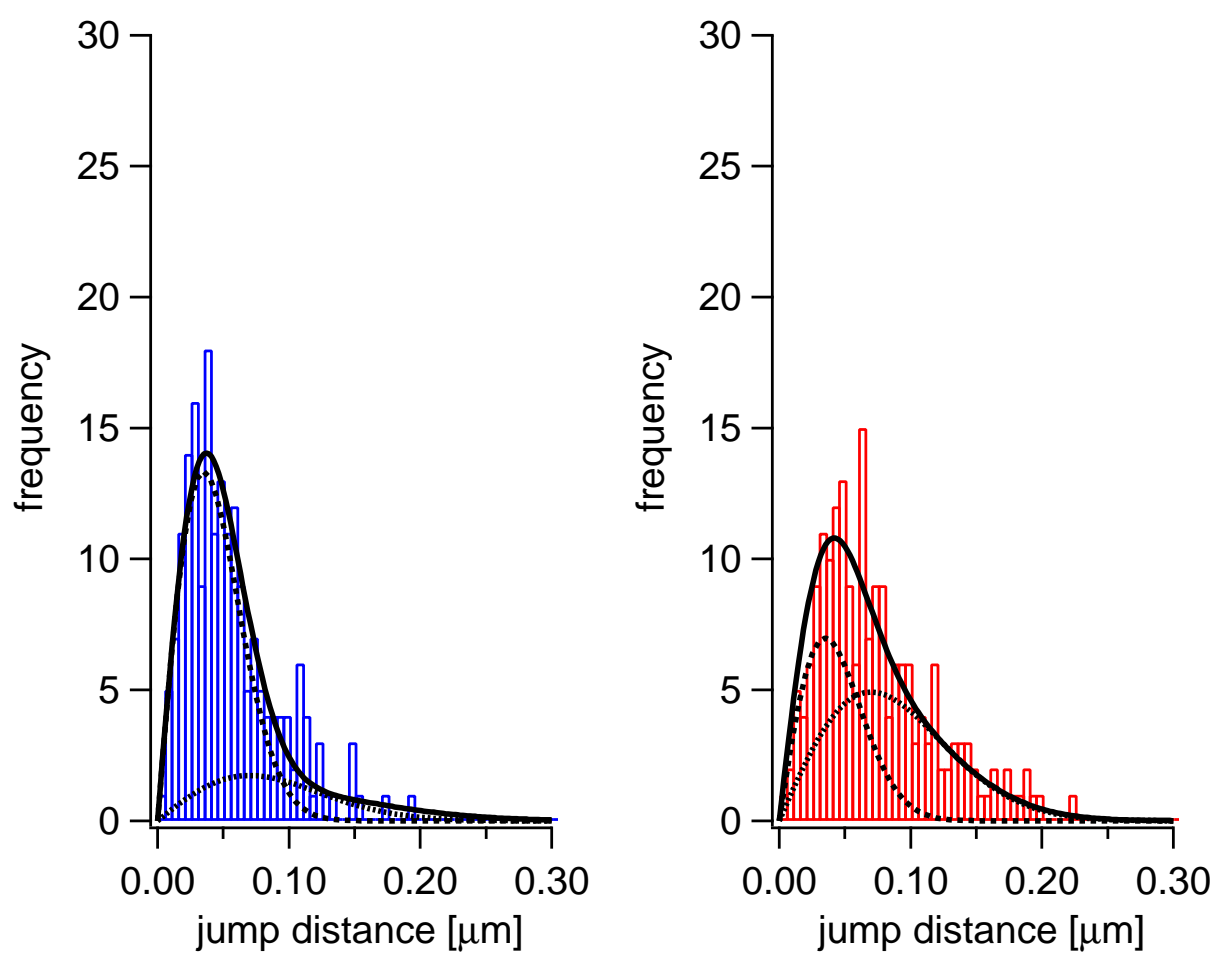

Figure 4.18: The left panel shows the jump frequency histogram of unstimulated vesicles for only $\mathrm{N}=194$ data points (compare to figure 4.16) and the two-component fit in black solid lines. The right panel shows the histogram analyzed for the same vesicles and the same number of jumps for the stimulated vesicles (see the above given examples for how frames were selected). The dashed and dotted lines highlight the two fractions of the two component fit.

Figure 4.19: K-S-test for mobility of rapidly endocytosed vesicles. Normalized jump frequency cdf of the stimulated vesicles (red) plotted vs the unstimulated vesicle jump frequency cdf. The blue diagonal shows the obtained plot in case of identical cdf's. The black lines show the 95\% confidence interval which is calculated according to the K-S theory to be $\pm 1.36 \cdot \sqrt{N}$, with $\mathrm{N}$ being the number of sample points (here $\mathrm{N}$ $=194$ ).

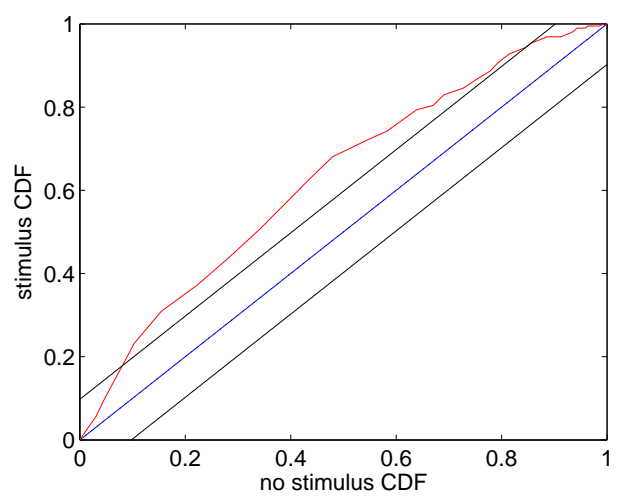




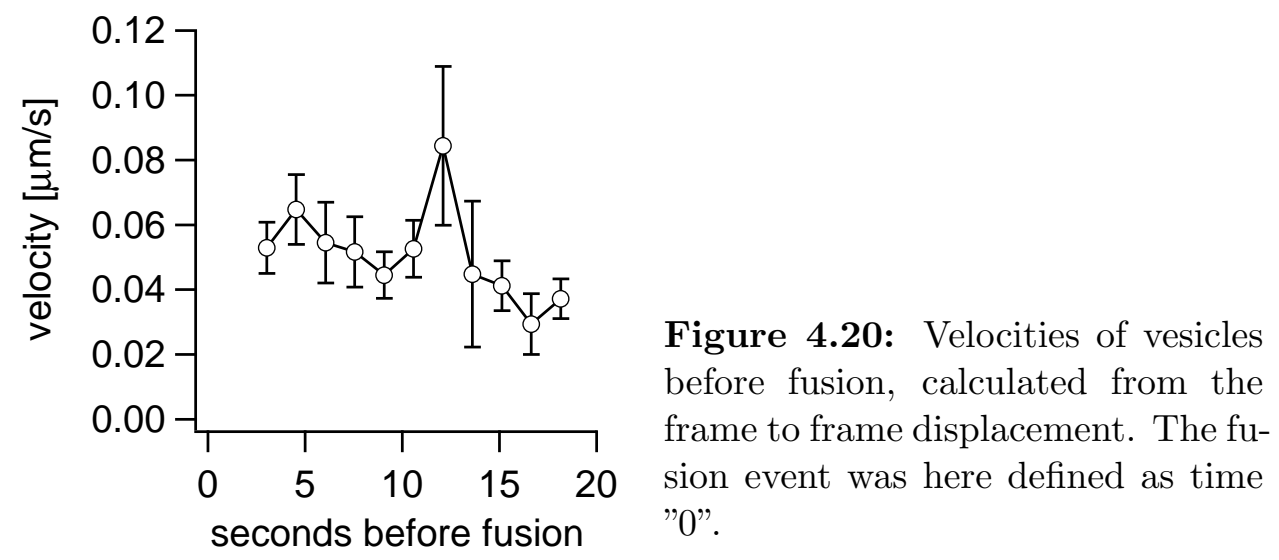

\section{Velocity of rapidly endocytosed vesicles}

It is certainly interesting to investigate when a vesicle is mobilized due to stimulation. The velocity was determined from the distance a vesicle moved from one frame to the next, i.e. within 1.512 s. In figure 4.20 the velocities are plotted versus a time axis, were the fusion time point was defined as the "0" time point. No abrupt increases in the velocities are visible and the average velocities did not exceed $0.1 \frac{\mu m}{s}$. The requirement to reject the last two frames before fusion from analysis (due to the departitioning rate of FM 1-43, see above and section 3.3.2 limits the analysis to approximately $3 \mathrm{~s}$ before the fusion event. In these three seconds vesicles could abruptly jump over long distances and thus increase their velocity, however the performed analysis suggests that vesicles are moving slowly to their release site.

\subsubsection{Slowly endocytosed vesicles}

After characterizing the dynamics of rapidly endocytosed vesicles, it was tempting to characterize dynamics of vesicles that were endocytosed differently. Since for the rapidly endocyotosed vesicles the dye was only briefly present during stimulation, two modes of endocytosis could have happened. A fast clathrin-mediated mechanism (suggested by Mueller et al. (2004)) or kiss and run, which has been suggested by Aravanis et al. (2003b). Murthy and Stevens (1999) used another stimulation protocol for minimal staining, which excludes the possibility that vesicles could be taken up by a rapid mechanism. The dye was applied several tens of seconds after the end of the stimulus, which only allowed for the staining of vesicles that were endocytosed via a slow mechanism, presumably via clathrin mediated endocytosis (Mueller et al., 2004). 

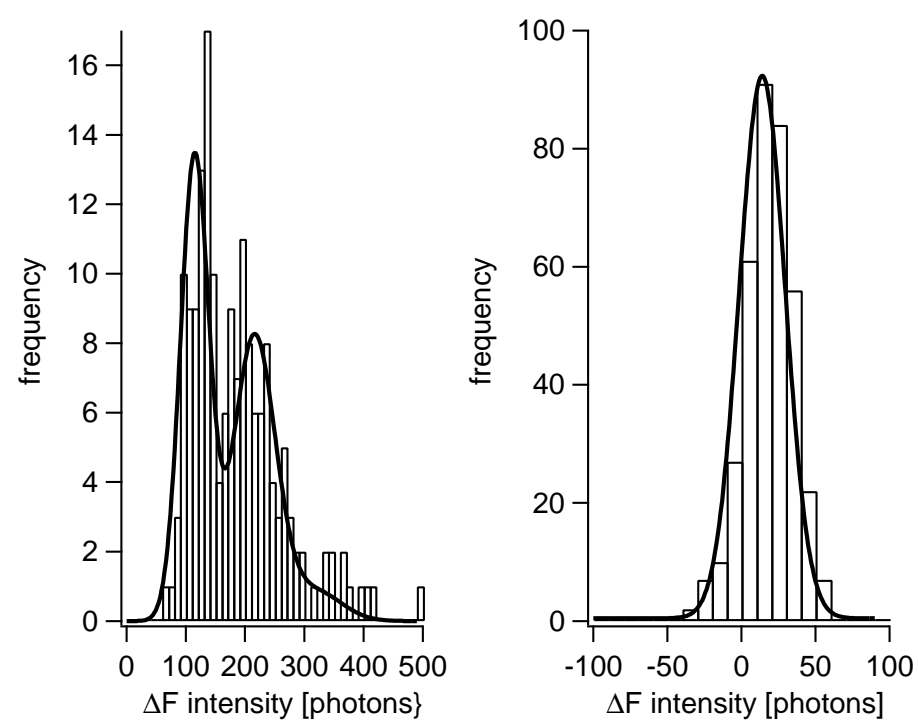

Figure 4.21: Slowly endocytosed vesicle histogram. The left histogram shows the quantal distribution from intensity measurements of 169 green fluorescent puncta. The black solid line shows a fit according to equation 2.1 and revealed the intensity of a single vesicle to be 100 photons, which is similar to the results in figure 4.11. The offset $\left(\mu_{o f f s e t}\right)$ due to bleed-through from the red into the green channel was determined to be 14 photons (right panel).

Please note that the analysis in this and the following two sections was performed in identical fashion as in the case of the rapidly endocytosed vesicles, so that its description will be somewhat shortened. Please refer to the preceding paragraphs for details where necessary.

To study the mobility of slowly endocytosed vesicles, the minimal staining protocol was slightly modified. The culture was stimulated with 100 APs @ 10 Hz (a total of $10 \mathrm{~s}$ ). During the stimulation no dye was present. The dye was then applied 30 seconds after the end of the stimulus for $10 \mathrm{~s}$. This guaranteed that only slowly endocytosed vesicle were stained. Figure 4.21 shows the obtained quantal histogram from measuring the intensity of single fluorescent spots. Also shown in this figure is the histogram from which the offset of the quantal histogram was determined.

Figure 4.22 summarizes the destaining kinetics of the fully stained synapses and the single vesicles, while figure 4.23 shows the distribution of fusion events as a function of time. The obtained results were similar to the results from the preceding section on rapidly endocytosed vesicles (compare with section 4.3.1, or see table 4.2). 

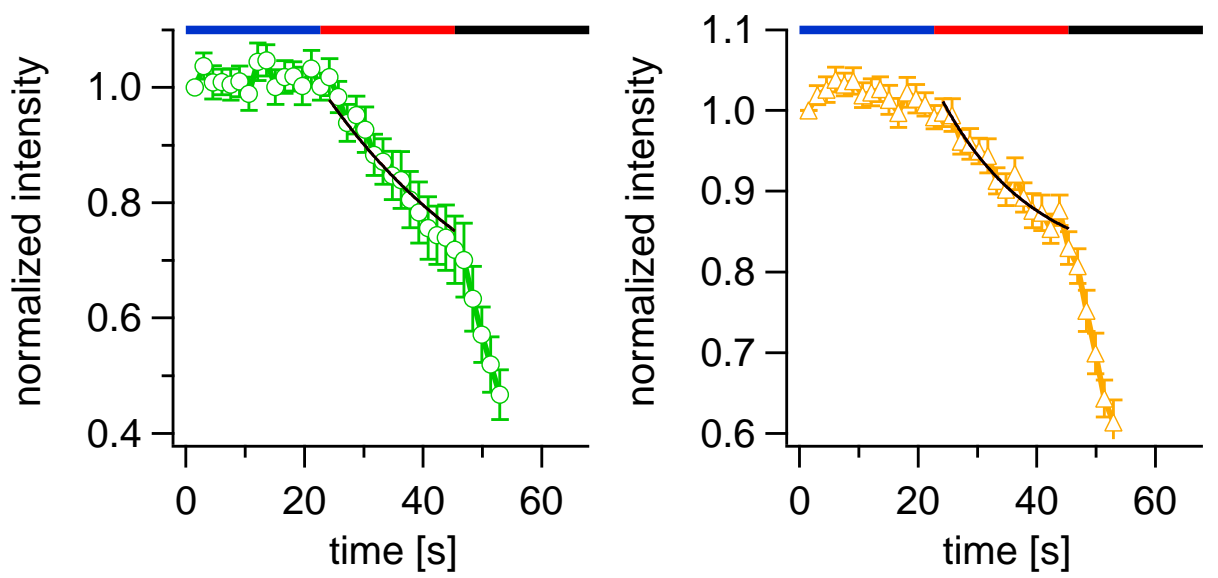

Figure 4.22: Destaining kinetics of slowly endocytosed vesicles and corresponding synapses. A mono-exponential was fitted for the time window where the weak stimulus of 75 APs @ $5 \mathrm{~Hz}$ was applied. The left panel shows the result for the average over the single vesicles and a fit with $\tau_{v e s}=34.5 \mathrm{~s}$ and the right panel for the synapse with $\tau_{\text {ves }}=15.6 \mathrm{~s}$.

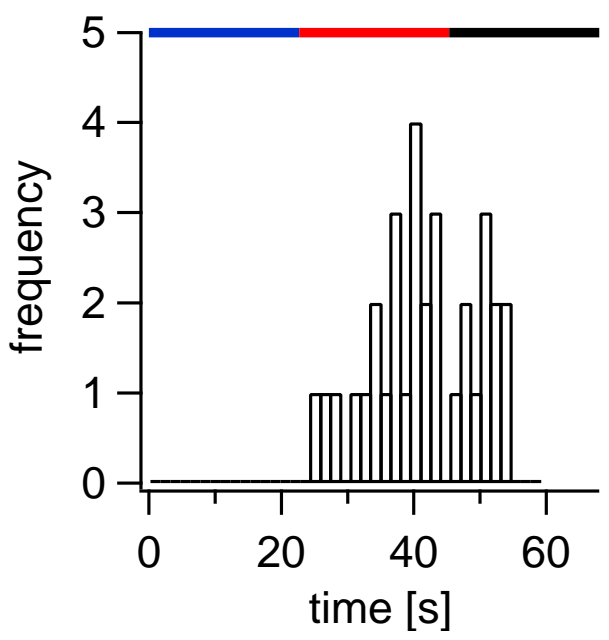

Figure 4.23: The histogram shows the distribution of fusion events over time. $69 \%$ of the 29 analyzed vesicles fused during the arrival of the first 75 APs. 

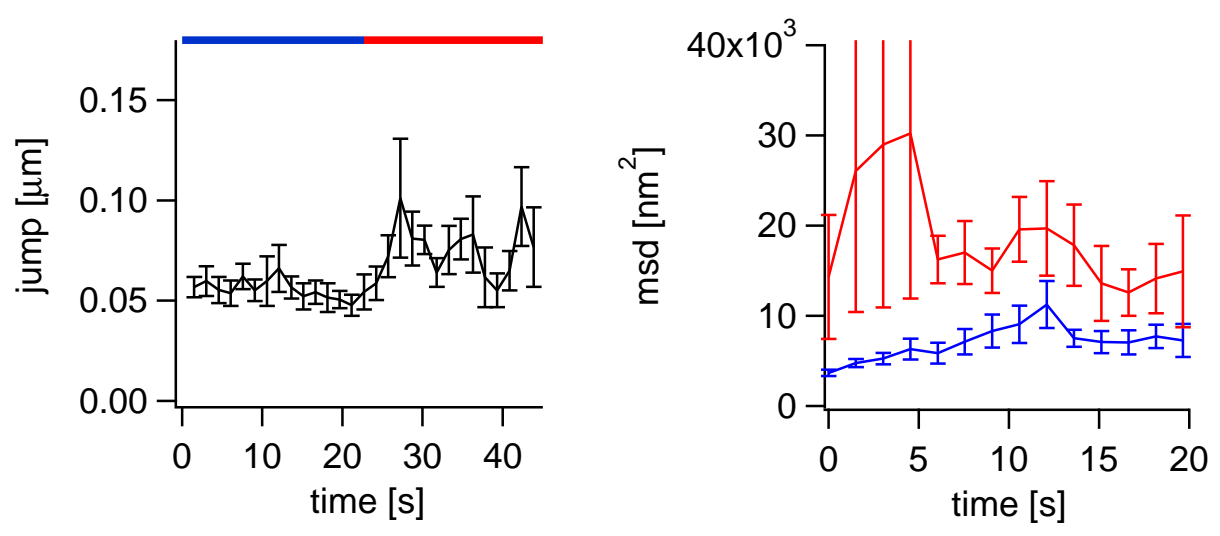

Figure 4.24: Average mobility and MSD analysis of slowly endocytosed vesicles. The left panel shows the average frame to frame jump distance for all analyzed slowly endocytosed vesicles. Beginning with the weak stimulation (indicated by the red bar) the mobility seemed to increase. The right panel shows the results form the MSD analysis on vesicle mobility before and during stimulation. The blue plot for the resting vesicles indicated confined diffusion, and the red MSD for the stimulated vesicles is very noisy, but suggests a mobility increase. Error bars represent s.e.m..

\section{Mobility analysis of slowly endocytosed vesicles}

Figure 4.24 summarizes the analysis of vesicle dynamics endocytosed via the slow pathway using overall mobility and MSD analysis. The performed analyzes were explained in detail in the preceding section. The overall mobility analysis indicated again a slight vesicle mobility increase upon stimulation. The MSD was saturating consistent with a model of caged diffusion. The MSD for the stimulated vesicles was very noisy and hard to interpret.

\section{Jump frequency distribution analysis of slowly endocytosed vesicles}

In the analysis of rapidly endocytosed vesicles, the jump frequency distribution analysis was a good tool to perform a quantitative analysis. The analysis was now repeated for the slowly endocytosed vesicles and is summarized in figure 4.25. Here, only one population of vesicle mobility could be identified for the unstimulated vesicles with $D=5.02 \cdot 10^{-4} \frac{\mu m^{2}}{s}(\mathrm{~N}=448$ of "all" frame to frame jump distances of unstimulated vesicles, frame 1-15) indicating that these vesicles were more or less immobile. In figure 4.25 the two distributions for unstimulated and stimulated vesicles are shown. The fit gave almost the same result for the unstimulated vesicles $(N=273$, see above how these 273 frames were picked out of all 448 jumps) as for the analysis of all 448 
jumps with $D^{\text {nostim }}=5.02 \cdot 10^{-4} \frac{\mu m^{2}}{\mathrm{~s}}$. For vesicle mobility during stimulation, the data was fitted with a one-component model returning $D^{\text {stim }}=8.92 \cdot 10^{-4} \frac{\mu m^{2}}{\mathrm{~s}}$ or with a two-component model with $f_{1}^{\text {stim }}=0.55, D_{1}^{\text {stim }}=5.32 \cdot 10^{-4} \frac{\mu m^{2}}{s}($ fixed $)$ and $f_{2}^{\text {stim }}=0.45, D_{2}^{\text {stim }}=1.976 \cdot 10^{-3} \frac{\mu m^{2}}{s}$. For easier comparison of fits to the different histograms, the one immobile component found for the unstimulated vesicles was fixed as the first component in this analysis. By means of the AIC (see figure 4.25) the two-component model was identified as the better model describing the mobility for slowly endocytosed vesicles under mild stimulation $(5 \mathrm{~Hz})$. The K-S analysis was then used to prove that the two distributions for unstimulated and stimulated vesicles were significantly different (see figure 4.25), KS-distance $=0.11$.

\section{Velocity of slowly endocytosed vesicles before fusion}

In figure 4.26 the average velocity of the single vesicles is plotted over the time before the fusion event.

\subsubsection{Spontaneous miniature events}

Even in the absence of any action potentials arriving at the synapse, vesicle are still undergoing spontaneous exo-endocytosis (Katz and Miledi, 1969). These events are called spontaneous miniature events, or in short: minis. Ryan et al. (1997) and Murthy and Stevens (1999) showed that single vesicles could be stained using the spontaneous firing rate of the synapse.

To investigate the mobility of vesicles that were endocytosed following a spontaneous fusion event, the minimal staining protocol was modified as follows. The dye was applied to the cultures for $30 \mathrm{~s}$ as opposed to the usual $10 \mathrm{~s}$ period. Unfortunately, longer exposure of the culture to the dye was not possible, since application of FM 1-43 resulted in non-synaptic staining of the culture, as for example by constitutive endocytosis of the underlying glia, which increased with the application time of the dye and thus lowered contrast. To be able to compare the final tracking data, it was necessary to ensure that the imaging quality was kept as constant as possible (refer to methods section 3.3 for details).

Over 40 coverslips were measured and only very few fluorescent spots originating from vesicles or vesicle clusters could be identified. The algorithm (see section 3.3 for details) identified less than three vesicles to be "trackable". Nevertheless, this result had a positive implication, too. It showed that the spontaneous release rate was too 

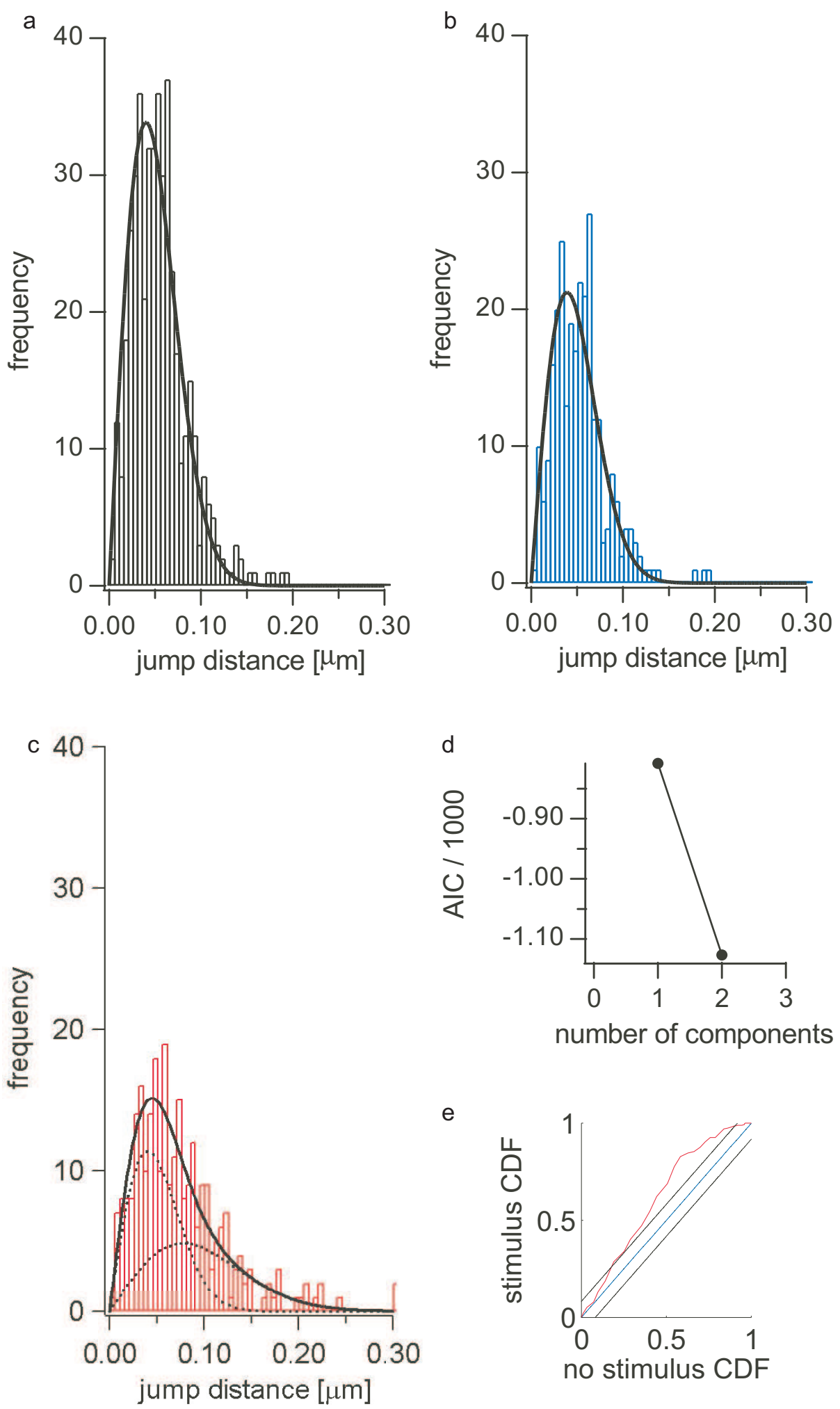

Figure 4.25: Jump frequency analysis of slowly endocytosed vesicles. Panel a shows the jump frequency histogram for all jumps under resting conditions and panel $\mathbf{b}$ only for the same number of jumps as analyzed for the stimulated conditions (c). The black lines show the corresponding fits with one component for $\mathbf{a}$ and $\mathbf{b}$ and two components for $\mathbf{c}$. Panel $\mathbf{d}$ shows the AIC analysis for a one-competent fit versus a two-component fit of the vesicle mobility during stimulation (c). Panel e shows the K-S analysis for the two distributions in panel $\mathbf{b}$ and $\mathbf{c}$. The red line representing the CDF from histogram c vs $\mathbf{d}$ does not lie within the $95 \%$ confidence bounds (black lines). 


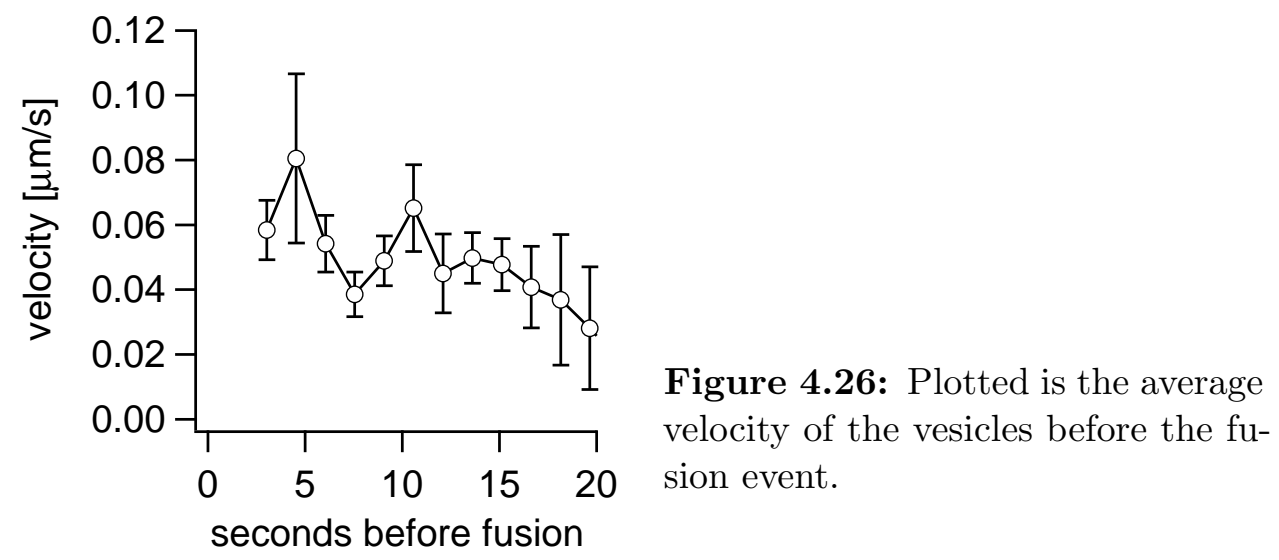

slow to significantly contaminate the results of the rapidly and slowly endocytosed vesicles.

Recently, Sara et al. (2005) investigated the kinetics of spontaneously recycling vesicles in hippocampal synapses. In their experiments the probability for a spontaneous fusion event was increased in high calcium solution (see appendix A for media and solutions). Increasing the mini frequency allows more spontaneously retrieved vesicles to be stained during a short application of dye.

\subsubsection{Mini staining using high $\mathrm{Ca}^{2+}$-solution}

Spontaneously retrieved vesicles were stained according to the following protocol: FM 1-43 was applied to the culture for $20 \mathrm{~s}$ in the presence of TTX, APV, CNQX and $8 \mathrm{mM} \mathrm{Ca}^{2+}$ (see appendix A for details on media and solutions). Tetrodoxin (TTX) blocks the generation of any action potential. More than 70 cultures were measured and the intensity histogram of 61 fluorescent puncta (figure 4.27) revealed the single vesicle intensity in these experiments to be 111 photons, comparable to the preceding experiments.

Since TTX is a high-affinity toxin, there was some concern that these experiments might not be comparable to the others due to a remaining effect of TTX on the excitability of the neurons. Analysis of the destaining kinetics of the fully stained synapses thus served as an excellent control and yielded $\tau_{\text {synapse }}=20.1 \mathrm{~s}$, a similar value as obtained before, verifying that TTX must have been washed out sufficiently (see figure 4.28). The destaining kinetics for the average over the 21 analyzed single vesicles $\left(\tau_{\text {ves }}=19.9 \mathrm{~s}\right)$ is also shown in figure 4.28 and the distribution of the fusion 

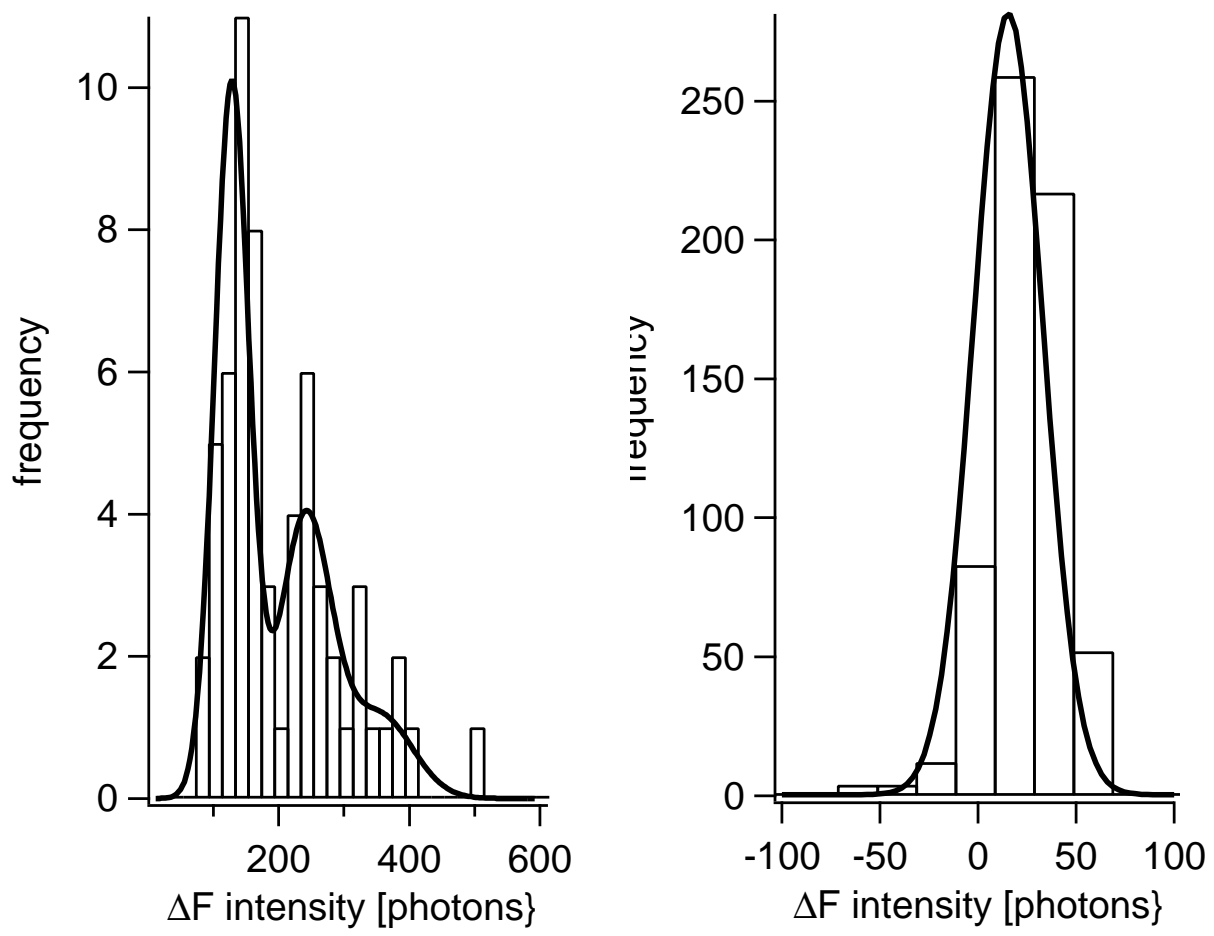

Figure 4.27: Intensity distribution from fluorescent spots of spontaneously stained cultures. The left panel shows the quantal intensity distribution of green fluorescent punctae $(\mathrm{N}=61)$. The solid black line is the fit according to the model described by equation 2.1 and determined the intensity of a single vesicle to be 111 photons. The right panel shows the histogram used to quantify the offset in the left histogram. A Gaussian fit returned $\mu_{\text {offset }}=15$ photons. 

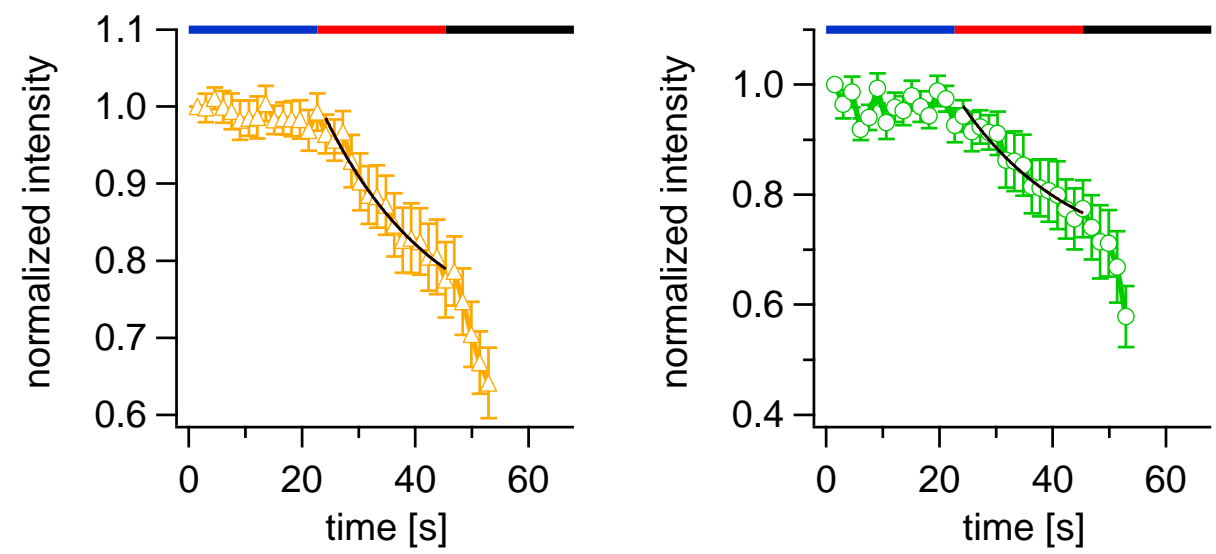

Figure 4.28: Destaining kinetics of 21 synapses (left panel) and their corresponding single vesicles (right panel). The black lines represent the result from a monoexponential fit to the data for the time points during weak stimulation.

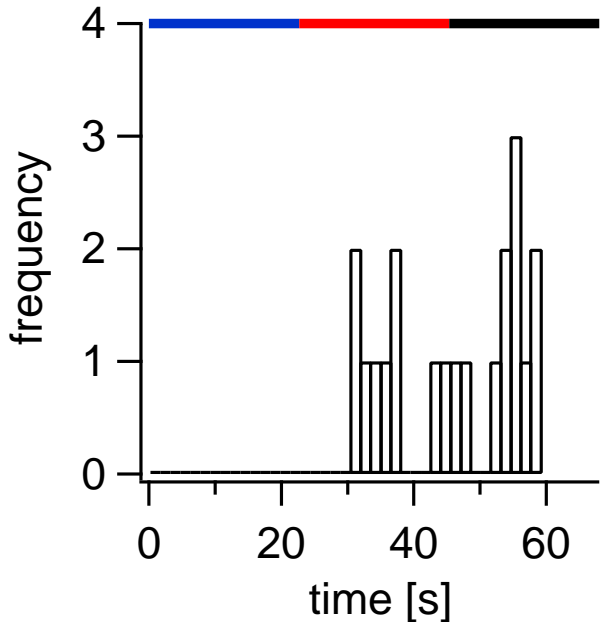

Figure 4.29: This plot shows the distribution of fusion events of the 21 vesicles. About $48 \%$ of them were released within the first 75 APs applied at $5 \mathrm{~Hz}$.

events in figure 4.29 .

Figure 4.30 gives an overview of the average mobility of single vesicles, which was found to be low. Also shown is the result from the MSD analysis, which resulted in a less noisy diagram for the stimulated vesicles and indicates mobilization of vesicles.

\section{Jump frequency distribution analysis for spontaneously recycled vesicles}

Figure 4.32 summarizes the results from the jump frequency analysis, which was performed in analogy to the preceding sections. For the mobility of spontaneously stained vesicles, all three histograms could be fitted better using a two component model. The AIC analysis in figure 4.32 was performed exemplarily on the jump frequency distribution for all jumps under resting conditions $(\mathrm{N}=294)$. In this instance 

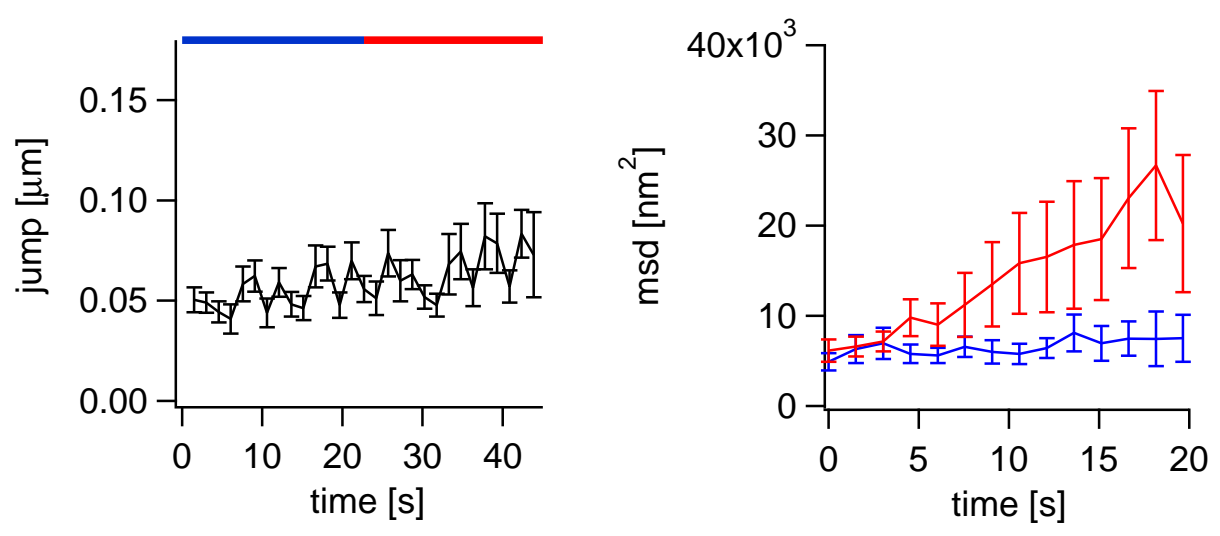

Figure 4.30: The left panel shows the average frame-to-frame jump distance for 21 analyzed single vesicles. The right panel shows the MSD for unstimulated vesicles in blue, and for the same vesicles during stimulation in red.

Figure 4.31: Plotted is the average velocity of single vesicles over the time before the detected fusion event.

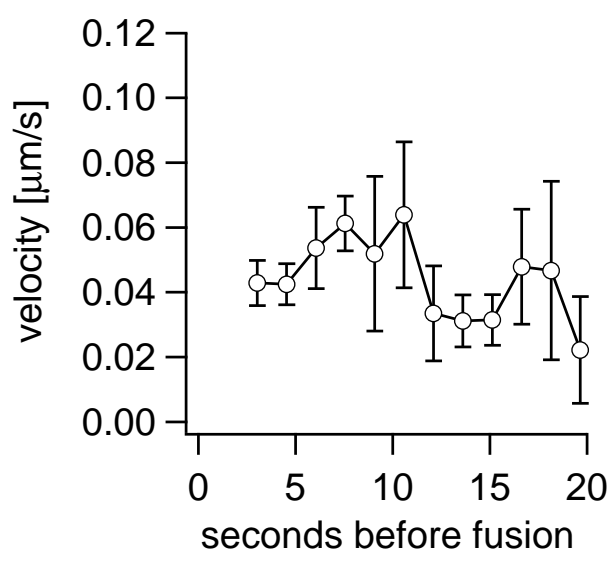

a three component model could be tested as well, but was found not to be a valid model for the data by means of the AIC. The two-component fit revealed a slow component with $f_{1}=0.85, D_{1}=4.60 \cdot 10^{-4} \frac{\mu m^{2}}{s}$ and a faster component with $f_{2}=0.15$, $D_{2}=2.80 \cdot 10^{-3} \frac{\mu \mathrm{m}^{2}}{\mathrm{~s}}$. The fit for vesicles during stimulation yielded $f_{1}^{\text {stim }}=0.76$, $D_{1}^{\text {stim }}=4.60 \cdot 10^{-4} \frac{\mu m^{2}}{s}($ fixed $)$ and $f_{2}^{\text {stim }}=0.24, D_{2}^{\text {stim }}=2.22 \cdot 10^{-3} \frac{\mu m^{2}}{s}$. The analysis of the same number of jumps for the unstimulated vesicles was very similar to the analysis of all jumps in absence of stimulation (see table 4.2). The K-S analysis of vesicles at rest and under stimulation in figure 4.32 suggested that the two distributions were only slightly different, since almost the complete CDF was within the $95 \%$ confidence bands. 

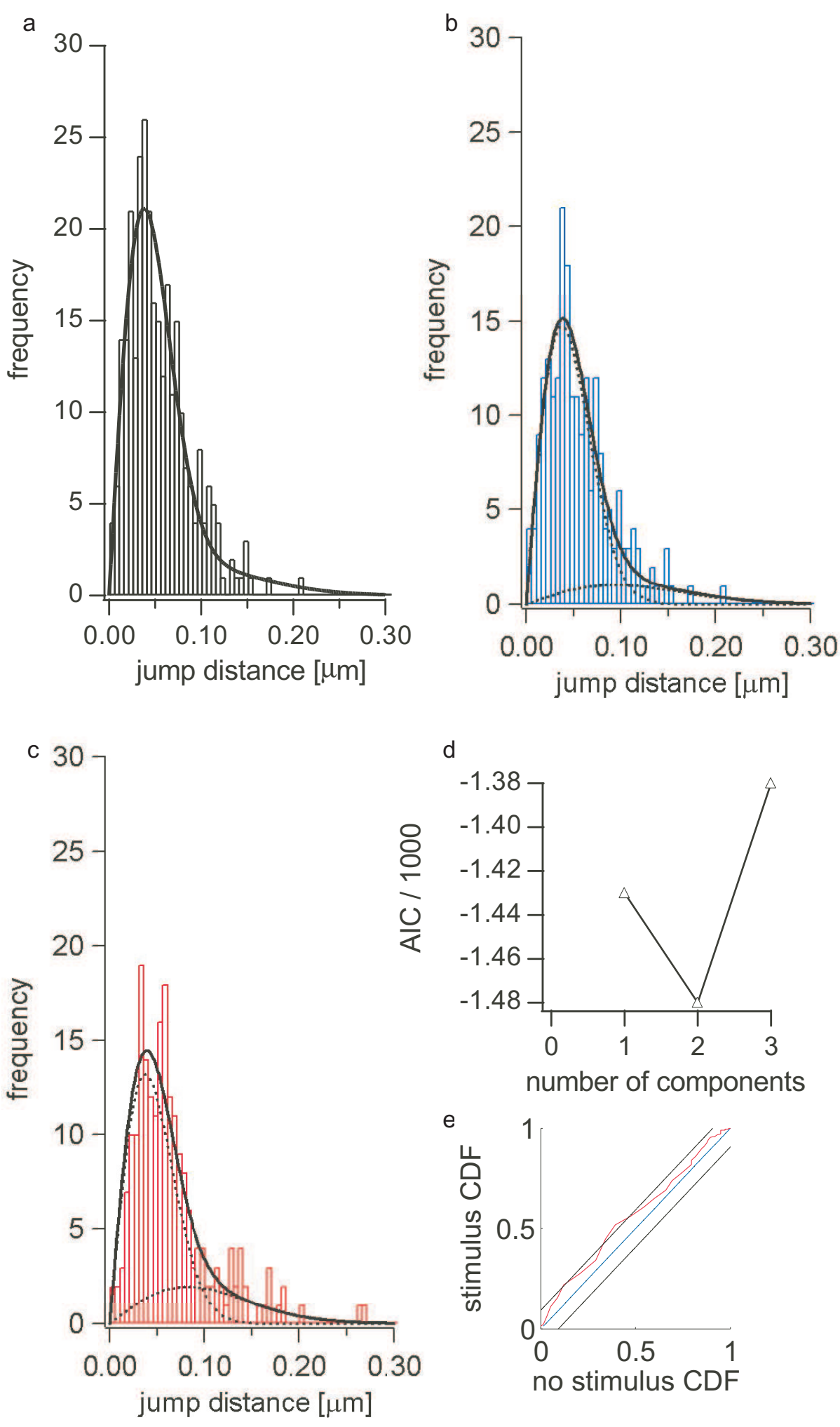

Figure 4.32: Jump frequency analysis of spontaneously recycled vesicles. Panel a,b and $\mathbf{c}$ show the jump frequency distributions of all jumps during resting conditions $(\mathrm{N}=$ 294 , a), and for $\mathrm{N}=214$ analyzed for the conditions before and during stimulation (b,c), as well as the corresponding two-component fits in solid black lines. Dashed and dotted lines visualize the underlying individual components. Panel $\mathbf{d}$ shows the results of AIC comparing one, two and three-component fits of the distribution in panel $\mathbf{a}$. The result from the K-S analysis of the distribution in $\mathbf{b}$ and $\mathbf{c}$ is plotted in panel $\mathbf{e}$. 


\section{Velocity of spontaneously endocytosed vesicles}

The average velocities of vesicles before the fusion event is analyzed in figure 4.31 .

\subsubsection{Summary of data on single vesicle tracking}

In the preceding sections, the mobility of single vesicles was determined using single particle tracking. The procedure in selecting and tracking vesicles ensured reproducibility. One should note that for each of the three different experimental conditions used for particle tracking studies, more than 70 coverslips were analyzed. Together with the vesicle intensity measurements from chapter 4.1.1, there are four independent experiments yielding a single vesicle intensity around 100 photons which strengthened confidence in the reproducibility of the experiments and showed that dye uptake by the vesicles was always quantized.

The destaining kinetics of the synapses can be taken as a control that experimental conditions were similar throughout the experiments. However, as already mentioned, one has to be careful when interpreting the destaining kinetics quantitatively, especially for the bright red fluorescent synapses, since these kinetics rely on intensity measurements (in the fast image acquisition mode) which might be biased due to the non-linear detection behavior of the avalanche photo diodes. Table 4.2 summarizes all results obtained from single particle tracking using the dual-color setup. 


\begin{tabular}{|c|c|c|c|c|c|}
\hline & & \multirow[b]{2}{*}{ unit } & \multicolumn{3}{|c|}{ endocytosis type } \\
\hline \multicolumn{2}{|l|}{ parameter } & & rapidly & slowly & mini \\
\hline $\begin{array}{l}\text { number of green fluores- } \\
\text { cent spots }\end{array}$ & $N_{\text {spots }}$ & \multirow{3}{*}{ photons } & 109 & 169 & 61 \\
\hline intensity of single vesicles & Int & & 91 & 100 & 111 \\
\hline $\begin{array}{l}\text { number of " trackable" sin- } \\
\text { gle vesicles }\end{array}$ & \multirow[t]{2}{*}{$N_{S V}$} & & 22 & 29 & 21 \\
\hline $\begin{array}{l}\text { fusion during weak stimula- } \\
\text { tion }\end{array}$ & & $\%$ & 68 & 69 & 48 \\
\hline synapse destaining & $\tau_{\text {synapse }}$ & $\mathrm{s}$ & 21.5 & 15.6 & 19.9 \\
\hline averaged vesicle destaining & $\tau_{\text {vesicle }}$ & $\mathrm{S}$ & 33.9 & 34.5 & 20.1 \\
\hline \multirow{2}{*}{$\begin{array}{l}\text { jump frequency analysis } \\
\text { for "all" vesicles under rest- } \\
\text { ing conditions } \\
\text { one component jump fre- } \\
\text { quency analysis }\end{array}$} & $N_{\text {frames }}$ & & 308 & 448 & 294 \\
\hline & $D$ & $\frac{\mu m^{2}}{s}$ & $5.12 \cdot 10^{-4}$ & $5.32 \cdot 10^{-4}$ & $5.02 \cdot 10^{-4}$ \\
\hline \multirow{4}{*}{$\begin{array}{l}\text { two component jump } \\
\text { frequency analysis }\end{array}$} & $f_{1}$ & $\%$ & 80 & --- & 85 \\
\hline & $D_{1}$ & $\frac{\mu m^{2}}{s}$ & $4.39 \cdot 10^{-4}$ & --- & $4.60 \cdot 10^{-4}$ \\
\hline & $f_{2}$ & $\%$ & & --- & 15 \\
\hline & $D_{2}$ & $\frac{\mu m^{2}}{s}$ & $1.74 \cdot 10^{-4}$ & --- & $28.79 \cdot 10^{-4}$ \\
\hline \multirow{5}{*}{$\begin{array}{l}\text { jump frequency analysis } \\
\text { for vesicles under resting } \\
\text { conditions }\end{array}$} & $N_{\text {frames }}^{\text {nostim }}$ & & 194 & 273 & 214 \\
\hline & $f_{1}^{\text {nostim }}$ & $\%$ & 83 & --- & 83 \\
\hline & $D_{1}^{\text {nostim }}$ & $\frac{\mu m^{2}}{s}$ & $4.39 \cdot 10^{-4}$ & $5.02 \cdot 10^{-4}$ & $4.60 \cdot 10^{-4}$ \\
\hline & $f_{2}^{\text {nostim }}$ & $\%$ & & --- & $28.64 \cdot 10^{-4}$ \\
\hline & $D_{2}^{\text {nostim }}$ & $\frac{\mu m^{2}}{s}$ & $29.50 \cdot 10^{-4}$ & --- & 17 \\
\hline \multirow{5}{*}{$\begin{array}{l}\text { jump frequency analysis } \\
\text { for vesicles during } \\
\text { stimulation } \quad \text { (fixed) }\end{array}$} & $N_{\text {frames }}^{\text {stim }}$ & & 194 & 273 & 214 \\
\hline & $f_{1}^{s t i m}$ & $\%$ & 46 & 55 & 76 \\
\hline & $D_{1}^{\text {stim }}$ & $\frac{\mu m^{2}}{s}$ & $4.39 \cdot 10^{-4}$ & $5.32 \cdot 10^{-4}$ & $4.60 \cdot 10^{-4}$ \\
\hline & $f_{2}^{s t i m}$ & $\%$ & & & 24 \\
\hline & $D_{2}^{s t i m}$ & $\frac{\mu m^{2}}{s}$ & $17.46 \cdot 10^{-4}$ & $19.76 \cdot 10^{-4}$ & $22.25 \cdot 10^{-4}$ \\
\hline KS-distance & & [a.U.] & 0.08 & 0.11 & 0.02 \\
\hline
\end{tabular}

Table 4.2: Summarized are the results from vesicle tracking experiments on rapidly endocytosed vesicles (rapidly, see section 4.3.1), slowly endocytosed vesicles (slowly, see section 4.3.2), and spontaneously endocytosed vesicles (mini, see section 4.3.4). 


\section{Discussion}

The motivation of this thesis was to gain new insights into the dynamics of vesicles inside hippocampal synapses. Vesicle mobility has hitherto not been studied very often in this system directly and in real-time. Hippocampal synapses are very small and densely packed with vesicles, so that studying the isolated movement of single hippocampal vesicles is experimentally very challenging. Rather, most of the knowledge about single vesicle dynamics is deduced from studies on larger systems, particularly the movement of large single granules in endocrine cells.

In the first part of this work, the fluorescence fluctuation spectroscopy (FFS) technique introduced by Jordan (2000) to the task of monitoring vesicle mobility in small synapses was expanded to a more quantitative tool using Monte-Carlo simulations. In the second part, a single particle tracking technique was developed, which allowed for direct observation of individual vesicles in real-time. In particular, different staining protocols were used to selectively label single vesicles taken up via rapid, slow and spontaneous endocytotic mechanisms. The mobility of vesicles was then investigated while the culture was at rest and during continuous stimulation of the synapse with action potentials at $5 \mathrm{~Hz}$.

Both techniques (FFS and single particle tracking) are in fact complementary to each other, and each have their pros and cons, which will be discussed below. During the technical development of the particle tracking project, a standard confocal fluorescence microscope equipped with avalanche photodiodes (APDs) was employed. A theoretical analysis of the detection performance of these APDs revealed that they are very well suited for single particle tracking studies, even though their detection performance is highly nonlinear. In the next paragraph the applicability of avalanche photodiodes for particle tracking experiments is discussed. 


\subsection{Performance of APDs in particle tracking experiments}

APDs are detectors that lack spatial resolution, and can thus only be used in fluorescence imaging when the spatial information is provided by scanning the image in a raster. Scanning microscopes are very popular in biology, so that, in principle, there would be ample opportunity to use APDs in particle tracking studies in biological systems. Furthermore, particle tracking studies have demonstrated great potential for investigating important biological phenomena; their applicability is currently still increasing and is expected to grow further in biology with the development of better fluorophores and microscopes. However, APDs are not commonly used in particle tracking experiments, probably since most researchers are reluctant to use a detector that has a dead-time, making it unable to detect photons some of the time, i.e. for a small period after a previous photon has been counted.

On the other hand, APDs were found to be one of the best available detectors with high apparent quantum efficiency (QE) at very low photon fluxes (Tan et al., 1999), which is the typical experimental paradigm in real-time fluorescent imaging, where it is usually desired to acquire images as long as possible with the highest possible sampling rate without significant bleaching of the fluorophores. It is thus unfortunate that these high QE detectors also have a dead-time. This gave the motivation to theoretically investigate how severely the dead-time effect jeopardizes the advantages of these detectors in particle tracking experiments.

Since the in-focus point spread function (PSF) in most standard fluorescence microscopes is approximately Gaussian-shaped, one convenient and widely used particle tracking algorithm is a direct Gaussian fit to the recorded diffraction image of a fluorescent subresolution particle. Data fitting requires some form of minimization procedure, where a model is continuously generated by the computer and compared to the raw data. Typically, finding the best unbiased model describing the data can be achieved by using Maximum Likelihood estimators. In chapter 3.2, different Maximum Likelihood (ML) estimators for fitting a Gaussian to a point spread function were tested. Most simulations were performed for the one-dimensional case since computation time increases drastically when simulating the two dimensional case (see below). 
First, different ML estimators for the case of an ideal counting process were tested. In ideal counting processes, the fluctuations in the detected signal can be described by Poisson statistics. To simulate this, a detected, "noisy" PSF was generated by drawing random numbers from a Poisson distribution with the expectation value at every pixel position given by the function describing the continuous ideal PSF (here, a 1D Gaussian). All fits were then performed on this "noisy" PSF. The tested ML estimators behaved as expected, and the best ML estimator derived from Poisson statistics was as good as theoretically expected from equation 3.5 , proving that the simulation was correct (see figure 3.4 and table 3.1).

Next, the simulation was performed assuming that the photon detection process was biased by the presence of $50 \mathrm{~ns}$ dead-time. Such a process is no longer following simple Poisson statistics. The ML estimator derived from dead-time biased counting statistics performed best, as expected, but surprisingly, the standard Poisson ML was almost as good in finding the true center of the PSF. Furthermore, the inverse variance (a measure of precision) no longer scaled linearly with the number of detected photons when these ML estimators were used, as shown in figure 3.6. The "value" of a detected photon for determining the center of the PSF increased at higher photon fluxes, and could no longer be predicted from equation 3.5. The reason for this effect is that at higher photon fluxes photons are not just lost due to the dead-time effect, but also the variance decreases relative to an ideal counting process (see figure 3.2). The "right" (dead-time) Maximum Likelihood estimator "knows" this additional information, and estimates the expected variance for every number of photon counts at a given pixel and weights this pixel accordingly in the minimization procedure.

The advantage of using an APD in particle tracking experiments is better explained in a more realistic example, when an APD with $40 \%$ quantum efficiency (QE) and a dead-time of $50 \mathrm{~ns}$ is compared with a detection device without dead-time, such as a PMT with 10\% QE. These are typical characteristics of commercially available APDs and PMTs. For simplicity, we assume that no further noise sources are present, but one should keep in mind that the strength of an APD is the fact that these detection devices introduce only very low additional noise to the detected signal. The following example is based on the results from simulations for the 2D case presented in section 3.2.6. Let us assume that a 2D PSF (originating from illumination of a subresolution particle) with an amplitude of $50 \frac{\text { photons }}{\mu s}$ is "arriving" on the APD. The average amplitude of the detected PSF would be $10 \frac{\text { photons }}{\mu s}$, corresponding to an inverse variance 
of $105.1 \frac{1}{p_{i x e l^{2}}}$. In contrast, if detected by the PMT, the detected amplitude would be $5 \frac{\text { photons }}{\mu s}$ and the inverse variance $32.3 \frac{1}{\text { pixel }^{2}}$, i.e. about $70 \%$ less. Thus, even at high photon fluxes the position estimate when using the APD is still much better than when using a standard PMT. Furthermore in the case of the APD the integral of the detected Gaussian was $533 \frac{\text { photons }}{\mu s}$. If a 2D Gaussian shaped PSF with an integral of 533 photons would be detected by a detector without dead-time (a hypothetical ideal detector with same QE), the inverse variance of the position estimate would be still $16 \%$ worse than with the APD, i.e. an inverse variance of $88.1 \frac{1}{\text { pixel }^{2}}$.

In many biological tracking experiments such high photon fluxes are not even present. In the study on single vesicle tracking in section 4.3 for example, the average photon flux at maximal amplitude of the 2D PSF was $\sim 2 \frac{\text { photons }}{\mu s}$. The lower the photon flux, the smaller the loss of photons due to dead-time, and the better the precision when using an APD. The described effect for dead-time affected counting processes is also present in the presence of background noise, as demonstrated in appendix E. However, the strength of the effect here depends on the signal to background ratio.

One should keep in mind that when using Poisson or dead-time Poisson ML estimators for minimization of a fit, as described in this thesis, it is important that the fit be performed on the raw data, since any form of filtering destroys the true counting statistics in the image. In the present study on vesicle tracking in neurons (section 4.3), the data was high-pass (and low-pass) filtered, since the background was highly inhomogeneous due to autofluorescence from underlying structures of dendrites and axons. Thus, the Poisson or dead-time ML could not be used, a $\chi^{2}$ estimator was used instead (see equation D.5). It remains to be tested in detail how severely the inhomogeneity would affect the usage of these estimators. However, in most non-neuronal studies, the background is more homogeneous, at least within a small region of interest around the fluorescent spot, which is usually sufficient for a good fit. This study suggests that APDs would be the superior detector for most tracking studies, even for bright objects; and having exceedingly bright objects is usually not the central problem in the field of live biological imaging. 


\subsection{A quantitative model for vesicle mobility from FFS experiments}

Jordan (2000) probed vesicle dynamics in hippocampal synapses using fluorescence fluctuation spectroscopy (FFS). It was the aim of the work described in section 4.1 to derive a quantitative model of vesicle mobility using Monte-Carlo simulations of the FFS data. For a proper simulation, one important parameter in the FFS experiments was still lacking from the experiments performed by Jordan (2000): a reliable estimate of the fluorescence intensity contribution from a single stained vesicle in the FFS experiments. This brightness was determined by combining fluorescence imaging and spectroscopy techniques, as outlined in detail in section 4.1.1. Monte-Carlo simulations in which the only unknown component was the vesicle mobility were then performed, generating simulated fluorescence fluctuation signals. These signals were analyzed the same way as the experimental data from Jordan (2000), i.e. using power spectral (PS), autocorrelation (ACF), mean and variance analysis.

Analysis of the PS was preferred, since interpretation of autocorrelation functions (ACFs) typically requires a priori knowledge and a fit with an analytical model, which was not available. Furthermore, the amplitude of the ACF also depends on the mean and on the variance of the fluorescence signal. The mean is a measure for the number of fluorescently labelled vesicles in the detection volume. The variance, however, a measure for the mobility of the objects in the detection volume was quite variable, even at similar mean intensities of the synapses, as shown in figure 4.1. A tighter correlation for the variance-over-mean ratio would be expected if the measurements on a single synapse could be repeated for stainings with different stimulus strength, which is, however, experimentally not feasible. For control cells, results of the Montecarlo simulations matched the experimental data, when it was assumed that vesicles are kept in place and can only diffuse in a small cage of approximately cage $_{\text {radius }} \sim$ $50 \mathrm{~nm}$ and a diffusion coefficient of $D=5 \cdot 10^{-5} \frac{\mu m^{2}}{\mathrm{~s}}$. These values are not unreasonable for a synapse, and similar results were also obtained for large granule motion in chromaffin cells by Steyer and Almers (1999) using particle tracking techniques where one type of motion was diffusion within a cage of cage $_{\text {radius }}^{\text {granule }}=70 \mathrm{~nm}$ and a $D_{\text {cage }}^{\text {granule }}=19 \cdot 10^{-4} \frac{\mu m^{2}}{\mathrm{~s}}$ or another type of motion was e.g. slow long distance motion with $D_{\text {long range }}^{\text {granule }}=1.88 \cdot 10^{-4} \frac{\mu m^{2}}{\mathrm{~s}}$.

The precision of the estimate for the diffusion coefficient and the cage radius de- 
pend on each other, as can be seen in the systematic simulation of FFS data in the appendix B. In the range of the diffusion coefficient for control cells, already a twofold change in $D$ could be detected by a change in the shape of the PS and in the variance over mean ratio. In contrast, changing the cage radius and fixing $D$ to $5 \cdot 10^{-5} \frac{\mu m^{2}}{\mathrm{~s}}$ only resulted in a small change of the variance over mean ratio and no change in the PS (see figure 4.6), so that for control conditions the cage radius $_{\text {lies }}$ between 25 and $100 \mathrm{~nm}$. The low sensitivity for the cage size parameter was a consequence of the high-pass filter (at $0.05 \mathrm{~Hz}$ ), which limited the data analysis to a 20 second time window. Thus, it cannot be concluded form this study that the only type of vesicle mobility in synapses is confined movement, but for a time window of $20 \mathrm{~s}$ this seems to be the most prominent type of mobility. Furthermore, it could not be distinguished whether the cage is indeed a physical cage due to e.g. an actin matrix, the space of one vesicle in the densely packed cluster, or the consequence of a tether attached to the vesicle that pulls it back to an origin if it moves too far.

Jordan (2000) also inhibited myosin activation by blocking the myosin light chain kinase (MLCK), and this data could be simulated assuming a 10-fold slower diffusion constant than in control cells (and same cage size, see figure 4.7). Thus, vesicle motion in resting synapses seems to stem from residual activity of myosins, while one can only speculate why this residual activity exists. Maybe myosins are part of a "street-cleaning" mechanism that actively organizes the synaptic vesicle cluster. The finding that disabling myosin function already decreases the $D$ of vesicles by a factor of 10 , even in resting synapses, points towards a much bigger importance of myosins for vesicle transport when the synapse is stimulated. This was also suggested by Ryan (1999) who could, however, only indirectly probe myosin importance by analyzing the kinetics of vesicle cycling at the synapse.

The broadband phosphatase inhibitor okadaic acid is implied to block the function of the phosphoprotein synapsin in living synapses. Results from experiments on okadaic-acid treated synapses were harder to reproduce with a model, indicating that in this case, caged diffusion is not the only type of mobility occuring on a timescale of $20 \mathrm{~s}$. The effect of okadaic acid increased with increasing concentrations of latrunculin B (Jordan, 2000). However, given that the pure okadaic acid effect was already difficult to model, modelling this additional effect was not within the scope of this thesis. In section 4.1.2, it was shown that the data can be approximated e.g. by assuming a 100-fold higher value for $D$ and cage $_{\text {radius }}=50 \mathrm{~nm}$ (relative to control conditions) 
for about $25 \%$ of the vesicles, or 8 fold higher $D$ than control for all vesicles and cage $_{\text {radius }}=25 \mathrm{~nm}$.

One additional piece of information can be obtained from the okadaic acid experiments. Alternatively to the presented model of caged diffusion, it is still conceivable to consider the model of mobile, freely diffusing vesicles and an immobile vesicle fraction as described in section 4.1 and by Jordan (2000). If one calculates the mobile fraction according to equation 4.2 for the okadaic acid data (see table 4.1), a mobile fraction of $2.3 \%$ for control synapses, and $5.1 \%$ for okadaic acid (OA) treated synapses is obtained (see table 4.1). First of all there is no evidence for a mobile fraction from FRAP studies on hippocampal synapses or frog NMJ (Kraszewski et al. 1996; Henkel et al., 1996). However, it could be also that the bleaching pulse was too long in the FRAP experiments, such that a mobile fraction was bleached during the bleaching pulse itself. Secondly, FRAP studies performed on frog neuromuscular junction (NMJ) pointed to a much stronger effect of okadaic acid (Henkel et al., 1996) than just a twofold increase in the mobile fraction. Finally, in the presented particle tracking study, which will be discussed in the next paragraph, also no mobile vesicles were found. Nothing seems to point into the direction of a mobile vesicle population, however, it cannot be excluded that there is a way to simulate the experimental data by assuming an immobile fraction and very few vesicles that diffuse in a bigger cage than $100 \mathrm{~nm}$, or even freely across the whole synapse. Nevertheless, the model derived from the performed Monte-Carlo simulations is physiologically reasonable, allowed to determine cage radii and diffusion coefficients, and could describe the experimental data both in resting boutons and for certain pharmacological treatments.

Summarizing all data obtained from the FFS study, vesicles are most likely anchored to actins via myosin and additionally to synapsin, as suggested in a current review from (Rizzoli and Betz, 2005).

\subsection{Single vesicle tracking}

"Watching" directly the mobility of a single vesicle is one of the most promising approaches to the study of vesicle dynamics. To achieve this for the study of vesicles within hippocampal synapses was the aim of the work presented in section 4.2 and 4.3 .

Particle tracking of individual single vesicles requires that the center of a fluores- 
cent spot originating from a single vesicle is precisely determined in every acquired image of a time series. As single stained vesicles are very dim compared to the background originating from autofluorescence and nonsynaptic staining, optical sectioning microscopy was used. Preliminary experiments with a two-photon setup, which consisted of merely tracking single vesicles, did not entirely demonstrate that the observed movements have their origin exclusively in vesicle movements, rather than movement of the whole synapse. Slow synapse movement is a well-known phenomenon in neurobiology (Fischer et al., 1998). Along with other technical problems in using the two-photon microscopy system, such as strong bleaching, this shortcoming led to the development of a dual-color single photon setup, where synapses (stained in red) and vesicles (stained in green) could be tracked simultaneously. This setup employed a standard confocal fluorescence microscope, where the pinhole was set to 1.7 Airy units, i.e. a compromise between reducing background and collecting enough fluorescence intensity from single stained vesicles for off-line particle tracking analysis.

Single synaptic vesicles are subresolution particles and a Gaussian fit was used for particle tracking. In contrast, synapses are bigger structures that can even vary in shape. These were tracked using a crosscorrelation algorithm. All region of interests where a shift of the synapse bigger than two pixel was detected were rejected from any further analysis. A shift of two pixels does not necessarily mean that indeed the synapse shifted, but could also be the consequence of the low precision of this algorithm (for details see section 3.3.2). It cannot be excluded that a very small mobility of synapses, such as jittering, still has a minor contribution to the detected mobility of vesicles. However, due to the described selection criterium, this would only cause a decrease in the precision limit of the single vesicle tracking algorithm.

Being able to image a single vesicle in green and the entire synapse with multiple stained vesicles in red also had several experimental advantages. Besides the fact that the experimental protocol was much shorter (compare the one-color and dualcolor protocol in figure 2.5), the chances for performing a good measurement were also much higher since "good" regions showing nicely spaced synapses and little background staining could be pre-selected in the red channel. However, the "pre-selection" procedure seemed to somewhat undermine the requirement for reproducible measurements: For particle tracking experiments under different biological conditions to be comparable, all analyzed vesicles must have been selected using the same selection criteria. To achieve this, the selection procedure was controlled by an automated 
algorithm that was presented in figure 3.10 .

Each particle tracking experiment consisted of two steps. First, the complete recycling pool of the synapse was stained with a red fluorescent styryl dye and strong stimulation of the synapse. Then, after washing of the culture a minimal stimulation protocol was applied to only stain very few vesicles per synapse with the green fluorescent FM 1-43. Next a time series was acquired. To monitor vesicle mobility during resting conditions and during stimulation of the culture, after acquisition of 15 frames the culture was weakly stimulated at $5 \mathrm{~Hz}$ for another 15 frames. Then another 3 times 600 APs were applied to the culture to release any releasable fluorescence from the synapse. The intensities of vesicles and synapses were analyzed before and after this destaining stimulus. From all vesicles that fulfilled certain criteria, such as the width of a subresolution particle, those were selected that had the intensity contribution from a single vesicle (for details of this procedure refer to section 3.3).

\section{Comparing vesicle mobility in the absence of and during action potential stimulation}

The particle tracking algorithm presented in section 3.3 was used to track the mobility of vesicles in the time series. One more problem was that the single vesicle intensity could change during stimulation of the culture. In order to be able to compare resting vesicles with those during stimulation, the algorithm halted when a vesicle lost $40 \%$ of its original intensity amplitude (determined by the Gaussian fit, see section 3.3). The coefficient of variation of the amplitude measurement was found to be roughly $c_{a} \sim 0.1-0.25$ (in the performed simulations, see table 3.2). Thus, a $40 \%$ drop of the fitted amplitude is very unlikely to be merely the consequence of the variance of the fitting routine. Furthermore, the $F W H M_{\text {axial }}$ of the PSF was $1200 \mathrm{~nm}$, so that a drop of $40 \%$ was also very unlikely to be the consequence of vesicle movement in the axial direction. On the other hand, if a vesicle lost less than $40 \%$ of its fluorescence intensity due to partial release of dye, the vesicle could still be tracked. Since Aravanis et al. (2003b) found that typically $>40 \%$ of the fluorescence is lost upon vesicle fusion, this could only happen in very rare cases. In summary, in most cases a drop of $40 \%$ in the Gaussian fit amplitude depicts the occurrence of a fusion event.

The designed particle tracking algorithm was then used to study vesicle mobility of rapidly, slowly and spontaneously endocytosed vesicles, which were fluorescently 
labelled using different minimal staining procedures as explained in subsections 4.3.1, 4.3 .2 and 4.3.4. The mobility of vesicles during resting conditions and during stimulation of synapses with a continuous train of action potentials at $5 \mathrm{~Hz}$ was analyzed.

\subsubsection{Mobility of single vesicles}

Diffusion coefficients for all three vesicle populations corresponding to three different endocytosis mechanisms (rapid, slow and spontaneous) were obtained by analyzing how far a vesicle jumped from one frame to the next (jump frequency analysis, see section 4.3.1. For resting vesicles, the value of $D$ lay around $D=4.4-5.3 \cdot 10^{-4} \frac{\mu m^{2}}{\mathrm{~s}}$, while for the rapidly and spontaneously endocytosed vesicles, a second component of $\sim 20 \%$ with roughly 4 -fold higher $D$ was found (see table 4.2 ).

Every tracking algorithm has a precision limit. Thus, even tracking a perfectly immobile particle would allow us to calculate a non-zero diffusion coefficient from a jump frequency distribution. This $D$ can be approximated from the standard deviation $\sigma_{P}$ of the position estimate by $D \cdot t=\frac{\sigma_{p}^{2}}{4}$. A $D$ of $4.5 \cdot 10^{-4}$ corresponds to a $\sigma_{P}^{\text {slow }}=52 \mathrm{~nm} .50 \mathrm{~nm}$ is the approximate precision of the Gaussian fit tracking algorithm (in the simulation the precision for an object with intensity of 100 photons was $\sigma_{P}^{\text {sim }}=40 \mathrm{~nm}$, see table 3.2 . The immobile component in the jump frequency distributions is thus not much different than the tracking precision. The true $D$ caused by vesicle movement is thus hidden in this $D$ and could be as low as zero. The results from the particle tracking experiment are thus not in disagreement with the FFS study, where the $D$ of resting vesicles was approximately a magnitude lower than the results of the jump frequency analysis.

During stimulation conditions, two components were found for all vesicle populations. Here the faster component accounted for roughly $50 \%$ for the rapidly and slowly endocytosed vesicles, and only for about $20 \%$ in the case of the spontaneously endocytosed vesicles. The difference between the jump frequency histogram of resting and stimulated vesicles was found to be significant. For the spontaneously stained vesicles, stimulation of synapses only resulted in a very minor increase in the faster component, and the histograms looked more similar, even though in terms of the Kolmogorov Smirnov (KS) analysis, they were significantly different (see figure 4.32). However, if one compares the KS plots for all three different vesicle populations, it is obvious that the differences are much stronger for the rapidly $(\mathrm{KS}$-distance $=0.08$ ) 
and slowly endocytosed vesicles $(\mathrm{KS}$-distance $=0.11)$ than for the spontaneously endocytosed vesicles $(\mathrm{KS}$-distance $=0.02$, see figures $4.19,4.25$ and 4.32$)$. If one calculates as described above the standard deviation for the $D$ of the more mobile component this gives for $D=22 \cdot 10^{-4} \frac{\mu m^{2}}{s}$ a $\sigma_{P}^{\text {faster }}=115 \mathrm{~nm}$. This component is higher than the precision limit, however, the calculated $D$ is still biased by the precision of the tracking algorithm, such that all values given for $D$ are only upper limits of $D$ 's of the true vesicle mobility.

The two components are two components of the jump distribution and thus do not necessarily represent two populations of vesicles. The exact physiological correlate of the two components remains somewhat speculative. The slow component found in the jump frequency distribution could maybe depict either vesicles or just states of a vesicle during its lifetime, where it is tightly tethered to a cytoskeletal element. During stimulation, this tether might be lost because vesicles are transported to their release site, and thus the $D$ increased. The $D$ of the more mobile component is still very low, and points to a highly organized vesicle transport machine, which suggests that the vesicles are not transported to the active zone over long distances. This is also evident from the fact that the velocity of vesicles does not seem to exceed $\sim 0.1 \frac{\mu m}{s}$ (see $4.20,4.26,4.31$ during stimulation.

The slow departitioning rate of FM 1-43 from the plasma membrane only allows to determine the exact time point of a fusion event with $\sim 3 \mathrm{~s}$ uncertainty. Dye that was already released from the vesicle but not yet washed away from the region of interest could lower the precision of the tracking algorithm and thus the last $3 \mathrm{~s}$ (2 frames) before a detected fusion event were excluded from analysis. It is thus theoretically still possible that in the last moment $(<3 \mathrm{~s})$ before the fusion event, the vesicles are transported very quickly over large distances. No evidence from this study, however, points in this direction.

\section{Vesicles move within a cage in the absence of stimulation}

A method to investigate the type of movement of a tracked particle is the mean square displacement analysis. Plotting the particle's mean square displacement over time typically yields a straight line for free diffusion, a saturating curve for confined diffusion, and a non-saturating curve for active transport.

The MSD analysis in figures 4.15, 4.24 and 4.30 shows that the movement was 
highly restricted and that the maximum amplitude of all three MSD plots for rapidly, slowly and spontaneously endocytosed vesicles was approximately $10000 \mathrm{~nm}^{2}$, corresponding to an approximate cage size $\sim 100 \mathrm{~nm}$. But as for the diffusion coefficient the amplitude of the MSD had an offset caused by the limited precision of the tracking algorithm, so that even at very high time resolution, the MSD would not start at zero but have a zero offset. Furthermore, due to the low time resolution, the first points that would normally show a decline of the curve towards zero or the zero offset in the MSD analysis are missing. Due to the lack of data points, an analytical fit was not performed, and the amplitude offset could also not be quantitatively analyzed. The $100 \mathrm{~nm}$ cage $_{\text {radius }}$ thus represents the upper limit of the true cage size; therefore, also the cage radius estimate is in good agreement with the results from the FFS experiments, where the cage radius was approximately $50 \mathrm{~nm}$.

The MSDs for vesicle mobility during stimulation were very noisy, but they suggested mobilization, i.e. that vesicle movement was no longer just restricted to confined diffusion.

\subsubsection{Destaining kinetics of single vesicles and synapses}

In addition to tracking the position of vesicles and synapses in every image of the time series, the absolute intensities were measured to analyze the destaining kinetics in the red and green channel over time. The setup employed avalanche photo diodes that show a nonlinear detection behavior already at photon fluxes exceeding $\sim 2 \frac{\text { photon }}{\mu s}$. The setup was optimized for the task of particle tracking, and not for measuring intensities in a fast acquisition mode. Thus, all absolute values of destaining kinetics obtained from time series recordings have to be interpreted with some caution (see figure $4.13,4.22$ and 4.28). This is especially important for the fully stained synapses monitored in the red channel, where the photon fluxes were higher than in the green channel. The purpose of the destaining analysis was to verify that synapses were stimulated in an approximately similar way throughout all experiments which was found to be the case (all $\tau_{\text {synapses }}$ lay between $15-22 \mathrm{~s}$, see table 4.2). Please note that for the purpose of analyzing absolute intensities of minimally stained boutons for the

histogram analysis (see figures 4.11, 4.21 and 4.27) images were acquired in a slow acquisition mode, where the pixel dwell time was long enough for the APDs to be operated in their linear range (see section 2.3.2). 


\section{Distribution of fusion events}

In addition to the analysis of the average destaining kinetics of synapses and vesicles, the distribution of fusion events of the single vesicles over time was analyzed (see figure 4.12, 4.23 and 4.29). For the rapidly and slowly endocytosed vesicles out of all detected fusion events for all vesicles about $70 \%$ fused during the first 75 APs of the weak $5 \mathrm{~Hz}$ stimulus and from the spontaneously endocytosed about 50\%. Aravanis et al. (2003b) also found that rapidly endocytosed vesicles have a very high release probability during weak stimulation of the neuron.

The number of sampled vesicles, however, is small, so that it is hard to determine if the difference between rapidly/slowly endocytosed vesicles and the spontaneously endocytosed vesicles is significant, or just a consequence of the low sample number. Furthermore, about $70 \%$ of the data on fast and slow endocytosed vesicles was obtained about 6 month earlier than the rest of the experiments. During this time, the method was slightly optimized (as for example the measuring chamber was changed) which could have introduced a very small systematic error, which was however not detectable in any of the control experiments, but could also contribute to the slight difference observed in the distribution of fusion events.

\subsubsection{What do rapidly and slowly endocytosed vesicles have in common?}

Rapidly and slowly endocytosed vesicles behaved similar in terms of their mobility and in terms of their destaining properties, which also promotes confidence in the reproducibility of the experiments. However, they were endocytosed via two retrieval mechanisms that differ in their speed and maybe even in the mechanism of endocytosis, i.e. kiss and run for the fast mode (Aravanis et al., 2003b) and clathrin-mediated for the slow mode (Mueller et al., 2004). However, the rapidly and slowly endocytosed vesicles had one thing in common: In both experiments, they were the last to be endocytosed. In the protocol for staining rapidly endocytosed vesicles, these were the first and the last at the same time. In the protocol for staining slowly endocytosed vesicles, the synapses were fist stimulated with 100 action potentials for $10 \mathrm{~s}$, and the dye was then added $30 \mathrm{~s}$ after the stimulation ended, so that most likely only the last vesicles endocytosed were stained with dye (Ryan et al., 1996b). As for the rapidly endocytosed vesicles, about $70 \%$ of all detected fusion events occured during the weak stimulation with $75 \mathrm{APs}$ at $5 \mathrm{~Hz}$ (see figure 4.12 and 4.23 ). This allows the conclusion 
that under the stimulation protocols used for staining rapidly and slowly endocytosed vesicles, those vesicles that were the last to be endocytosed will always go out first when the synapse is stimulated again. The last endocytosed vesicles might thus be the one that are targeted to the readily releasable pool. A recent study from Mueller (2004) also pointed in this direction.

The spontaneously endocytosed vesicles behaved a bit differently than the other two vesicle populations studied. They seemed to be less activated by stimulation in terms of mobility increase, as well as in the probability to fuse during arrival of the first 75 APs. The last point would be in line with the finding by Sara et al. (2005). However, this question is certainly very interesting and should be investigated in more detail. Particle tracking experiments performed with a higher time resolution and for a larger number of vesicles could certainly verify whether vesicle mobility of spontaneously endocytosed vesicles is different from the rapidly and slowly endocytosed ones, and whether their release probability during action potential stimulation is indeed lower.

\subsection{Imaging vs FFS; pros and cons and the future of the techniques}

Compared to particle tracking, FFS experiments can be peformed much faster. Less synapses had to be sampled to obtain reasonable results, while for particle tracking, more than 70 coverslips had to be measured to end up with about 20 individual vesicles for each condition. Furthermore, FFS does not require exceptionally high contrast images since synapses are stained more strongly. Certain pharmacological studies might not even be possible in the imaging experiments. For example, preliminary studies with okadaic acid showed that there is slight increase in autofluorescence due to incubation with this drug. This is of no concern for the spectroscopy technique, but it certainly is for the particle tracking, where it is necessary that all regions of interest have similar signal-to-background ratio. FFS typically requires that the average number of fluorescent particles stays constant, and thus limits this technique to resting conditions. However, a more interesting question in neurobiology is how a vesicle behaves in response to stimulation, and what types of movements drive a vesicle to the active zone. Particle tracking is ideally suited to answer these ques- 
tions. Theoretically and also practically, the time resolution can be increased, e.g. by scanning a smaller image region, or by using a more advanced scanning system that allows for faster acquisition of large regions (Tan et al., 1999).

If one were to use a dye that departitions from the membrane more quickly than FM 1-43, such as FM 2-10, it would no longer be necessary to drop the last frames before the fusion event from the analysis in the particle tracking experiments. The study could then be extended to monitor vesicle mobility immediately before the fusion event. However, the quantum yield of FM 2-10 is much lower than that of FM $1-43$, thus the biggest limitation is the quality of the dye. This problem may be solved in the near future, since the field is currently progressing very fast in developing more photostable dyes with a high quantum yield. Eventually, the FM 1-43 dye might also be replaced with some form of quantum dot.

While the potential of FFS is somewhat limited (as it may not be possible to apply this technique to the study of synapses during stimulation) the power of the particle tracking technique is theoretically only minimally limited. Speaking optimistically, and aside from the current technical limitations, the particle tracking assay presented in this thesis has the potential to monitor vesicle mobility similar to the study of Zenisek et al. (2000), who acquired more than 500 images from one synaptic terminal stained with FM 1-43 of the goldfish retina at very high time resolution using TIRF. One difference to the TIRF experiments certainly will remain, which is the unknown orientation of the active zone relative to the optical axis in hippocampal cultures, while in the TIRF experiments, the active zone was facing the coverslip, at least when fusion events could be observed. The single particle tracking technique in hippocampal synapses presented in this work is, however, limited to those experimental protocols where single vesicles can be stained in a quantal fashion, so that the intensity of a fluorescent spot makes it possible to identify the presence of one or more stained vesicles in the synapse.

In summary, the conclusion from both studies is that the synaptic vesicle cluster is highly organized, and that random movement of vesicles across the whole synapse, such as found in ribbon synapses of the goldfish retina (Holt et al., 2004), is not an abundant form of movement. This is in line with the result of FRAP studies on hippocampal synapses where no highly mobile vesicles could be detected (Kraszewski et al. 1996). Even during stimulation, the vesicle mobility is only slightly increased, 
making it very likely that active transport of vesicles in the hippocampal synapse is the main mechanism that drives a vesicle to its release site.

Both methods are valuable quantitative tools that can help to identify the molecular underpinnings regulating vesicle dynamics. Compared to other methods, these two assays selectively and directly probe only the vesicle movement. In contrast, FRAP, which also directly probes vesicle mobility, is very limited in its applicability to hippocampal synapses, since typically half of the synapse has to be bleached, while the total number of vesicles that can be stained is already very low. In fact, so far no quantitative analysis of vesicle mobility in hippocampal synapses has been performed with this technique.

Using the methods described in this thesis, it should be possible to close the gap in our knowledge concerning the mobility of small synaptic vesicles in small hippocampal synapses, as opposed to large granule mobility in endocrine cells, where particle tracking studies are widely applied in combination with e.g. TIRF and/or confocal microscopy. 


\section{A Media and solutions}

\section{A.1 Hippocampal cell culture}

Coating for coverlsips Matrigel (Beckton-Dickinson, BD 35 6239); diluted 1:50 in minimal essential medium (MEM 51200-038, Invitrogen-Gibco, Karlsruhe, Germany) containing 2.5 g glucose, $100 \mathrm{mg} \mathrm{NaHCO}_{3}$, $50 \mathrm{mg}$ transferrin (Calbiochem, Darmstadt, Germany) per $500 \mathrm{ml}$ medium.

Plating medium $100 \mathrm{ml}$ medium containing 10\% FCS (fetal bovine serum, Biochrom AG, Berlin, Germany), $1 \mathrm{ml} 0.2$ M L-glutamine (Sigma, Munich, Germany), $200 \mu l$ insulin solution.

Growth medium $100 \mathrm{ml}$ medium containing 5\% FCS, $0.25 \mathrm{ml} 0.2 \mathrm{M}$ L-glutamine solution, 2 ml B-27 supplement (Invitrogen-Gibco, Karlsruhe, Germany), $4 \mu M$ or 2 $\mu M$ cytosine arabinoside (ARA-C, Sigma, Karlsruhe, Germany).

Hank's solution Hank's balanced salt solution without calcium or magnesium (Sigma, Karlsruhe, Germany) plus 238 mg HEPES (1-Piperazineethane sulfonic acid, 4-(2hydroxyethyl)-monosodium salt), $350 \frac{\mathrm{mg}}{\mathrm{l}} \mathrm{NaHCO}_{3}, \mathrm{pH} 7.4$.

Dissociation solution Hank's solution plus $12 \mathrm{mM} \mathrm{MgSO}_{4} \cdot 7 \mathrm{H}_{2} \mathrm{O}$. Furthermore $1 \mathrm{mg}$ DNase (type IV, Sigma, Karlsruhe, Germany) was freshly added per $2 \mathrm{ml}$ dissociation solution.

Digestion solution $137 \mathrm{mM} \mathrm{NaCl}, 5 \mathrm{mM} \mathrm{KCl}, 7 \mathrm{mM} \mathrm{Na} \mathrm{HPO}_{4}, 25 \mathrm{mM}$ HEPES, $\mathrm{pH}$ 7.4. Furthermore $10 \mathrm{mg}$ Trypsin (type XI) and $1 \mathrm{mg}$ DNase (type IV, sigma, Karlsruhe, Germany) were freshly added per $2 \mathrm{ml}$ digestion solution. 


\section{A.2 Measuring solutions}

$150 \mathrm{mM} \mathrm{NaCl}, 5 \mathrm{mM} \mathrm{KCl,} 2 \mathrm{mM} \mathrm{MgCl}_{2} 10 \mathrm{mM}$ HEPES (pH 7.4), $30 \mathrm{mM}$ glucose (modified Tyrode solution) plus

stimulation solution $10 \mu M$ CNQX, (6-Cyano-7-nitroquinoxaline-2,3-dione) and 50 $\mu M$ APV (D,L-2-amino-5-phosphonovaelric acid (Tocris, Northpoint Forthway, UK) and $2 \mathrm{mMCaCl}$.

high $\mathrm{Ca}^{2+}$ solution $10 \mu M$ CNQX, $8 m M C a C l_{2}$ plus $1 \mu M$ tetrodoxin (TTX).

minimal $\mathrm{Ca}^{2+}$ washing solution $10 \mu \mathrm{M}$ CNQX and $0.1 \mathrm{mMCaCl} \mathrm{Cl}_{2}$ plus $2 \mathrm{mM}$ $\mathrm{MgCl}_{2}$. 


\section{B Systematic simulation of FFS data}

Monte-Carlo Simulations were performed as described in method section 3.1. For each cage radius four simulations were carried out with varying diffusion constants. The following effects can be observed (see figure B.1 and table B.1).

1. For low diffusion coefficients ( $D \leq 5 \cdot 10^{-5} \frac{\mu m^{2}}{s}$ ) the shape of the power spectra stays roughly constant and the variance increases only weakly with the cage radius. The reason for this is that very slow components are removed from analysis by high pass filtering $(0.05 \mathrm{~Hz}$, compare with figure 4.6).

2. For $D \geq 5 \cdot 10^{-4} \frac{\mu m^{2}}{s}$ the amplitude in the higher frequency range increases with cage size, as well as the variance. 

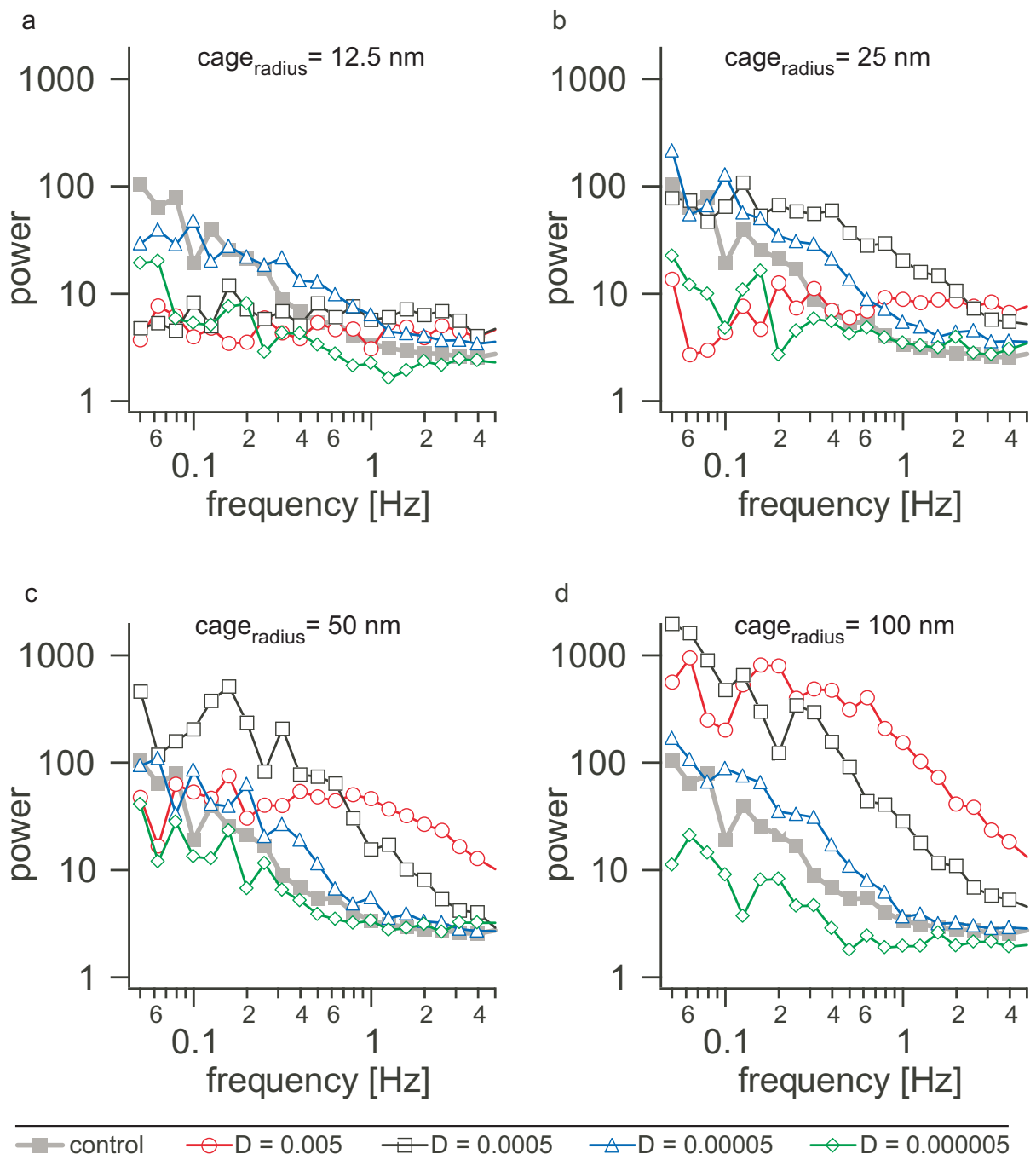

Figure B.1: In each panel the results from simulation of four different D's is shown. As a reference a measured trace from control cells is shown in grey in every picture. Panel $\mathbf{a}$ is a simulation for cage $e_{\text {radius }}=12.5 \mathrm{~nm}, \mathbf{b}$ for cage $_{\text {radius }}=25 \mathrm{~nm}, \mathbf{c}$ for cage $_{\text {radius }}=50 \mathrm{~nm}$ and $\mathbf{d}$ for cage $_{\text {radius }}=100 \mathrm{~nm}$. 


\begin{tabular}{|c|l|c|c|}
\hline cage $_{\text {radius }}[\mathrm{nm}]$ & $\mathrm{D}\left[\frac{\mu \mathrm{m}^{2}}{\mathrm{~s}}\right]$ & mean & variance \\
\hline \hline \multirow{5}{*}{12.5} & 0.005 & 15.7 & 0.5 \\
& 0.0005 & 18.3 & 0.6 \\
& 0.00005 & 16.7 & 0.5 \\
& 0.000005 & 11.7 & 0.1 \\
\hline \multirow{5}{*}{25} & 0.005 & 14.1 & 2.1 \\
& 0.0005 & 17.5 & 2.6 \\
& 0.00005 & 17.4 & 1.5 \\
& 0.000005 & 16.3 & 0.2 \\
\hline 50 & 0.005 & 12.6 & 6.3 \\
& 0.0005 & 11.3 & 5.4 \\
& 0.00005 & 13.1 & 1.1 \\
& 0.000005 & 16.5 & 0.2 \\
\hline \multirow{5}{*}{100} & 0.005 & 14.2 & 23.0 \\
& 0.0005 & 16.9 & 14.8 \\
& 0.00005 & 13.3 & 1.5 \\
& 0.000005 & 9.85 & 0.2 \\
\hline
\end{tabular}

Table B.1: Simulations were performed for different cage sizes and diffusion coefficients. Column three and four list the mean and variance of the fluorescence signal respectively. The respective power spectra are shown in figure B.1. 


\section{Optimal setup for single vesicle tracking}

\section{C.1 Bleaching of FM 1-43}

For exciting FM 1-43 a two-photon laser operating at $\lambda=900 \mathrm{~nm}$ and the $\lambda=488 \mathrm{~nm}$ from an argon laser were suitable. To ease focussing of the culture, the two photon laser was underfilling the back pupil of the objective, which resulted in a resolution decrease mainly along the optical axes.

Synapses were stained with FM 1-43 and the two-photon and argon laser were adjusted such that the synapses appeared in comparable brightness. This was done for a high power setting of the laser and a low power setting of the laser. The high powers setting of the argon laser was also used for the time series recordings in the vesicle tracking experiments described in section 4.3. The low power setting of the two photon laser was the same power used in the tracking experiments using this laser (section 4.2.1). Figure C.1 shows, that FM 1-43 dye was bleached much stronger using the multi photon laser. Table C.1 summarizes the data from the bleaching experiments.

\section{C.2 The optimal detector}

Two different highly sensitive photon counting heads were available (see figure 2.7 for details). To identify the photon counting head with the better performance (quantum efficiency) a $1 \mathrm{mM}$ Rhodamin $6 \mathrm{G}$ solution was scanned and the fluorescent light was split equally and detected by an APD and the Hamamatsu photon counting head (for detector details see figure 2.7). Figure C.2 plots the obtained count rate for the two detectors over the laser intensity. The slope for the APD is steeper and thus the APD is the more sensitive detector. 


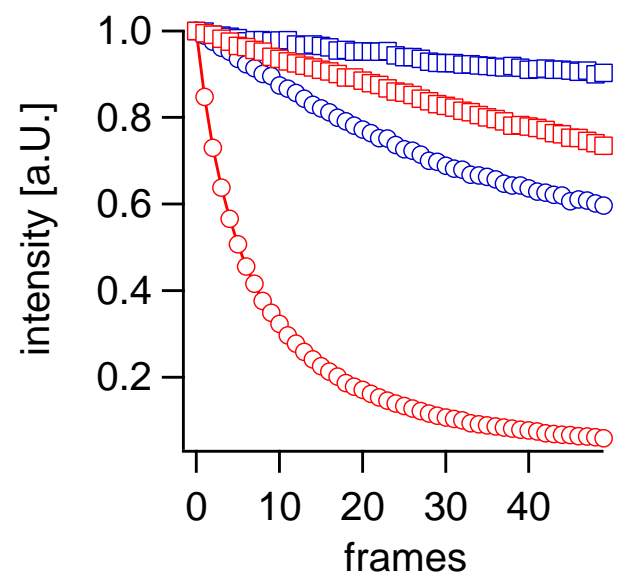

Figure C.1: Synapses stained with FM 1-43 were imaged using either two photon excitation (circles) or the $\lambda=488 \mathrm{~nm}$ from an argon laser (squares). The laser intensity was adjusted such that the synapses appeared with similar brightness. The bleaching was then measured at two different settings: High power of the two lasers are shown in red and low power settings in blue. The pixel dwell time was $1 \mu \mathrm{s}$. Please see the right table for data summary.

\begin{tabular}{|l|c|c|c|c|}
\hline & $\begin{array}{c}\lambda \\
{[\mathrm{nm}]}\end{array}$ & $\begin{array}{c}\text { power } \\
{[\mu W]}\end{array}$ & $\begin{array}{c}\text { Synapse } \\
\text { Int [a.U.] }\end{array}$ & $\begin{array}{c}\tau \\
\text { frames }\end{array}$ \\
\hline$M P_{\text {high }}$ & 900 & $13.4 \cdot 10^{3}$ & 90 & 9.8 \\
$A r_{\text {high }}$ & 488 & 26 & 100 & 159.4 \\
$M P_{\text {low }}$ & 900 & $6 \cdot 10^{3}$ & 14 & 85.1 \\
$A r_{\text {low }}$ & 488 & 4.5 & 17 & 433.4 \\
\hline
\end{tabular}

Table C.1: This table summarizes the data shown in the left plot. The PSF of the two photon excitation $(M P)$ was in the xy plane $F W H M_{x y}=520 \mathrm{~nm}$ and axial, $F W H M_{z}=$ $1900 \mathrm{~nm}$ (detected on the descan port of the microscope with an open pinhole, see figure 2.7 for details). For the single photon excitation $(A r)$ the PSF was $F W H M_{x y}=360 \mathrm{~nm}$ and $F W H M_{z}=1200 \mathrm{~nm}$. The pinhole was set to 1.7 airy. (FWHM= full width half maximum of a Gaussian distribution). The PSF was determined by measuring $40 \mathrm{~nm}$ green fluorescent (505/515 fluospheres, Molecular probes, Eugen, OR) beads settled on a cell layer. The pixel dwell time was $1 \mu s$. 


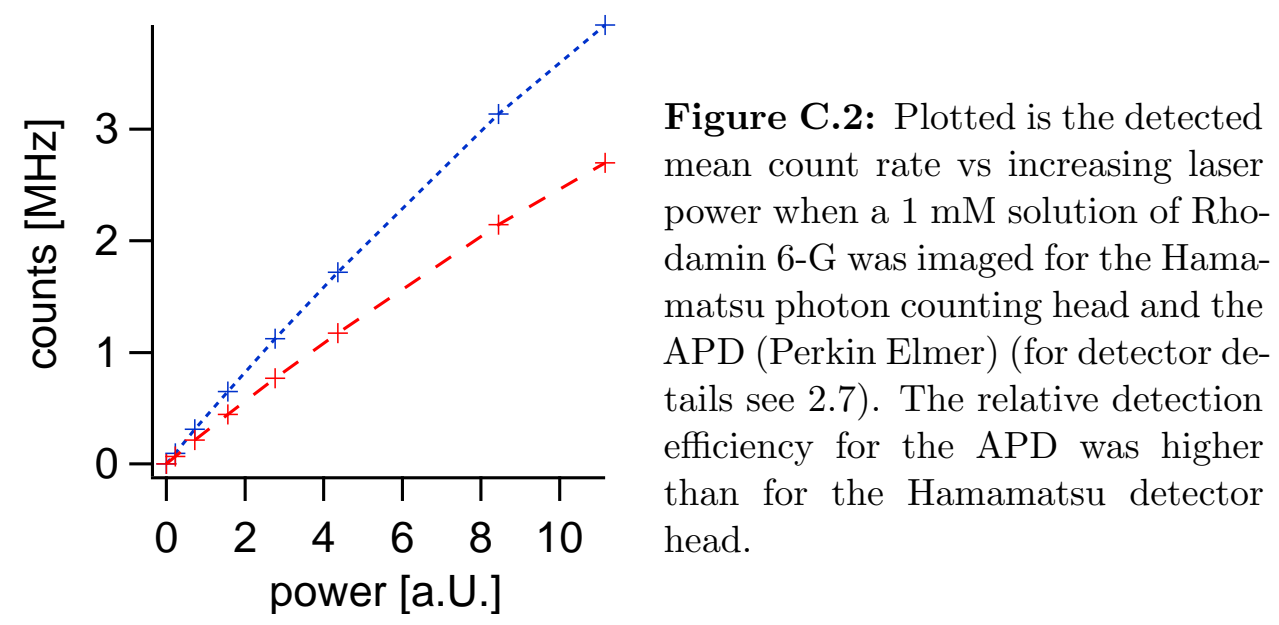

To increase the linearity of the detector unit, on each channel the light was split onto two APDs (for details see figure 2.7). Since the microscope was only equipped with two digital counters for each channel, the TTL pulses from each two APDs were shortened to $6 \mathrm{~ns}$ and combined. This basically created one detector unit with reduced dead-time. Figure C.3 summarizes the variance dependence from the mean count rate for all used detectors and detector combinations. The two APDs from Perkin Elmer showed the best performance (best linearity in the beginning) and were used to detect green fluorescent light, while the two APDs from EG\&G were employed on the red fluorescent channel. 

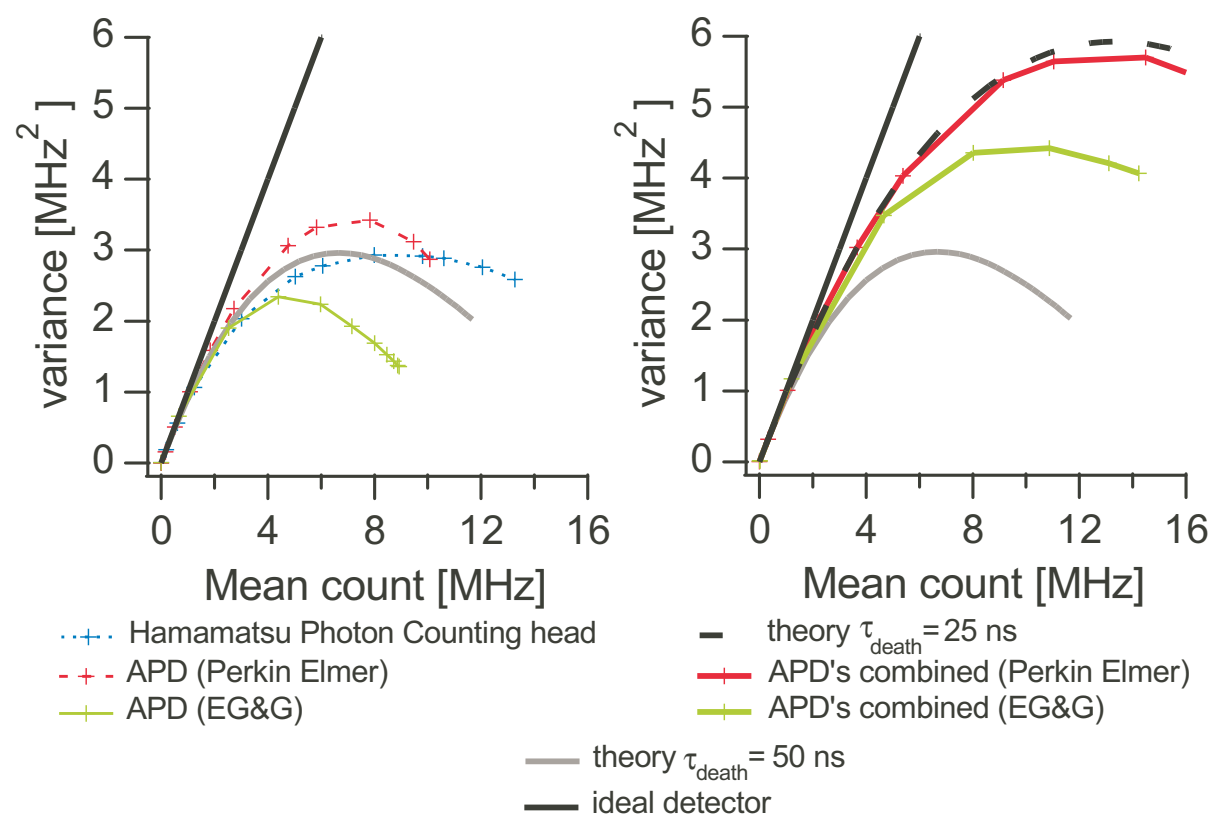

Figure C.3: The left panel shows the variance over mean plot measured for the individual photon counting detectors. The right panel shows the counting characteristics for each two APD's were the TTL pulses were first shortened to $6 \mathrm{~ns}$ and then combined using the custom-made pulse combiner from Picoquant (see figure 2.7, for details about detectors and pulse combiner). Shown are also the theoretical plots that were calculated from equation 3.1 and 3.4 . The theoretical curve with $t_{d}=25 \mathrm{~ns}$ approximates the experimental curve for the two combined Perkin Elmer APD's. 


\section{Position determination with non ideal photon detectors}

\section{D.1 Dead-time random number generators}

For ideal photon counting detectors the Poission number generator that is included in the "DIPimage" software package (available for MATLAB) was used. In the case of dead-time effected photon counting, two new random number generator were developed in MATLAB. MATLAB offers the possibility to pick random numbers from any given cumulative distribution (cdf) with the command "min(find(cumsum(cdf) $>$ rand(1)))1". The cumulative distribution was calculated from the dead-time distribution, see equation 3.3 .

Another way of generating a dead-time affected Poisson number generator, is possible since the expectation value $\mu$ of a Poisson distribution is the same as num times the expectation value of a Poisson distribution with mean $\frac{\mu}{n u m}$. This fact was used to compute a dead-time affected random number generator which will be referred to as the "bin dead-time number generator". For a given mean $\mu$, the probability was divided into 1000 bins. These bins can be assumed to be oriented along a time axes, such that each bin represents a time resolution of 1 ns. If a photon arrives in a bin, the next 50 bins are blanked corresponding to a dead-time of $t_{d}=50 \mathrm{~ns}$. Both random number generators were tested at intensities varying from $\mu=1-20$ each 10000 times and the mean and variance were calculated. Figure D.1 shows the variance over mean plot from these random number generators. As one can see, the dead-time random number generator generated via the "bin method" resulted in slightly better random numbers than the one derived via the cumulative distribution functions, which is most likely due to numerical problems. 
Figure D.1: The black line shows the ideal variance over mean plot calculated form equations 3.1 and 3.4. The blue trace was generated using the "bin" random number generator which matched the theoretical plot better than the dashed red trace, which shows the result of the cumulative distribution random number generator.

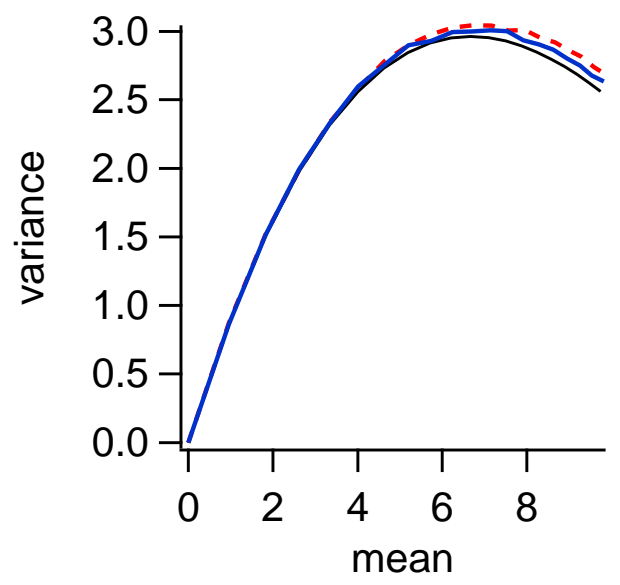

\section{D.2 Maximum Likelihood estimators}

Fitting a model to a data set requires to minimize the differences between the model and the data. This is typically done with the help of Maximum Likelihood (ML) estimators (Cramer, 1958). These estimators help one to determine the best model fit to the data that is the model with the maximum likelihood.

Fitting a model to an image $n_{i}$ that is the number of events counted in the time interval $\Delta t$ at every pixel $i$, aims on determining $\mu_{i}^{\prime}$ that is the mean intensity at pixel $i$ distributed according to a certain probability law. For a point process following Poisson statistics the following product of Poisson probability density distributions for the entire data set (image) has to be maximized

$$
P_{\text {Poisson }}\left(n_{i} \text { events in }(t, t+\Delta t) ; \mu_{i}^{\prime}\right)=\prod_{i=1}^{N} e^{-\left(\mu_{i}^{\prime} \cdot \Delta t\right)}
$$

where $\mu_{i}^{\prime}$ is the only parameter that is determined by the fitting routine. For simplicity the time interval is in the following absorbed into $\mu_{i}=\mu_{i}^{\prime} \cdot \Delta t$ giving

$$
P_{\text {Poisson }}\left(n_{i} ; \mu_{i}\right)=\prod_{i=1}^{N} e^{-\mu_{i}} \frac{\mu_{i}^{n_{i}}}{n_{i} !} .
$$

For probability density functions such as Gaussian, Gamma and Poisson, it is usually easier to minimize the log likelihood function instead. The only fitting parameter $\mu_{i}$ can than be determined by either maximizing $\mathrm{D} .2$ or minimizing

$$
\chi_{\text {Poisson }}^{2}=-\ln P_{\text {Poisson }}=\sum_{i=1}^{N}\left(\mu_{i}-n_{i} \cdot \ln \mu_{i}+\ln \left(n_{i} !\right)\right) .
$$


Since only $\mu_{i}$ will change during the minimization procedure, constant offsets and $\mu_{i}$ - independent factors can be dropped, leaving one with

$$
\chi_{\text {Poisson }}^{2}=\sum_{i=1}^{N}\left(\mu_{i}-n_{i} \cdot \ln \mu_{i}\right) .
$$

Analogously the Maximum Likelihood estimator for a product of Gaussian probability density function (pdf) $P\left(n_{i}, \mu_{i}, \sigma_{i}\right)=\prod_{i=1}^{N} \frac{1}{\sqrt{2 \pi \sigma_{i}}} \cdot \exp \left(-\frac{\left(n_{i}-\mu_{i}\right)^{2}}{2 \sigma_{i}^{2}}\right)$ is

$$
\chi_{\text {Gaussian }}^{2}=\sum_{i=1}^{N}\left(\frac{\left(n_{i}-\mu_{i}\right)^{2}}{\sigma_{i}^{2}}\right)
$$

after dropping constants offsets and $\mu_{i}$ - independent factors. This ML estimator will be refereed to as the Gaussian ML estimator, when the mean is equal the variance, such that $\sigma_{i}^{2}=\mu_{i}$.

If $\sigma_{i}^{2}$ is a constant, the denominator is $\mu_{i}$ - independent and can be dropped. This results in a formulation that is well known as a least square, or so called chi square $\left(\chi^{2}\right)$ estimator

$$
\chi^{2}=\sum_{i=1}^{N}\left(n_{i}-\mu_{i}\right)^{2}
$$

\section{D.2.1 ML estimator for a dead-time biased distribution}

The equation for the probability density function describing a dead-time biased photon counting process was given in equation 3.3. Similar to the above section we can calculate the ML estimator for the entire data set to be

$$
\chi_{t_{d}}^{2}=\sum_{i=1}^{N}\left(-\ln \left[\sum_{j=0}^{k_{i}=n_{i}} \cdot \frac{\mu_{i} \cdot\left(1-t_{d} \cdot k_{i}\right)^{j}}{j !}-\sum_{j=0}^{k_{i}=\left(n_{i}-1\right)} \cdot \frac{\left.\mu_{i} \cdot\left(1-\left(k_{i}-1\right) \cdot t_{d}\right)\right)^{j}}{j !}\right]\right)
$$

with $n_{i}$ being the actual measured value at pixel $i, t_{d}$ the dead-time and $\mu_{i}$ the value estimated by the fitting routine. To minimize numerical problems when calculating this equation this can be rewritten as 


$$
\begin{aligned}
\chi_{t_{d}}^{2} & =\sum_{i=1}^{N}\left(-\mu_{i}\left(t_{d} k_{i}-1\right)-\ln \left[\frac{\mu_{i}\left(1-t_{d} k_{i}\right)^{k_{i}}}{k_{i} !}\right.\right. \\
& \left.\left.+\sum_{j=0}^{k_{i}=n_{i}} \frac{\mu_{i}\left(1-t_{d} k_{i}\right)^{j}-e^{-t_{d} \mu_{i}}\left[\mu_{i}\left(1-t_{d}\left(k_{i}-1\right)\right]^{j}\right.}{j !}\right]\right)
\end{aligned}
$$

This equation is referred to as the dead-time Maximum likelihood estimator $\left(t_{d}\right.$-ML estimator) in the text.

Figure D.2 shows exemplar probability density functions for Poisson, Gaussian and dead-time function.

\section{A modified Gaussian ML estimator for dead-time affected counting processes}

For a dead-time biased photon detection process the mean and the variance for the detected photons can be calculated from the number of arriving photons on the detector according to equation 3.1 and 3.4 . One can now use these equations to create a modified Gaussian ML estimator, where the mean $\mu_{i}$ and the variance $\sigma_{i}^{2}$ determined by the fitting routine in the Gaussian ML estimator (see equation D.4) are replaced with these equations, giving

$$
\chi_{\text {Gaussian-PSF }}^{2}=\sum_{i=1}^{N}\left(\frac{\left(n_{i}-\hat{\mu}_{i}\right)^{2}}{\hat{\sigma}_{i}^{2}}\right),
$$

with $\hat{\mu}_{i}=\frac{\mu_{i}}{1+\mu_{i} \cdot t_{d}}$ (equation 3.1 and $\hat{\sigma_{i}^{2}}=\frac{\mu_{i}}{\left(1+\mu_{i} \cdot t_{d}\right)^{3}}$. This ML estimator will be referred to as the modified Gaussian ML estimator.

\section{A modified Poisson ML estimator for dead-time affected counting processes}

Similar to the preceding paragraph also a modified Poisson estimator can be calculated, that is

$$
\chi_{\text {Poisson-PSF }}^{2}=\sum_{i=1}^{N}\left(\hat{\mu}_{i}-n_{i} \cdot \ln \hat{\mu}_{i}\right),
$$

with $\hat{\mu_{i}}=\frac{\mu_{i}}{1+\mu_{i} \cdot t_{d}}$. 


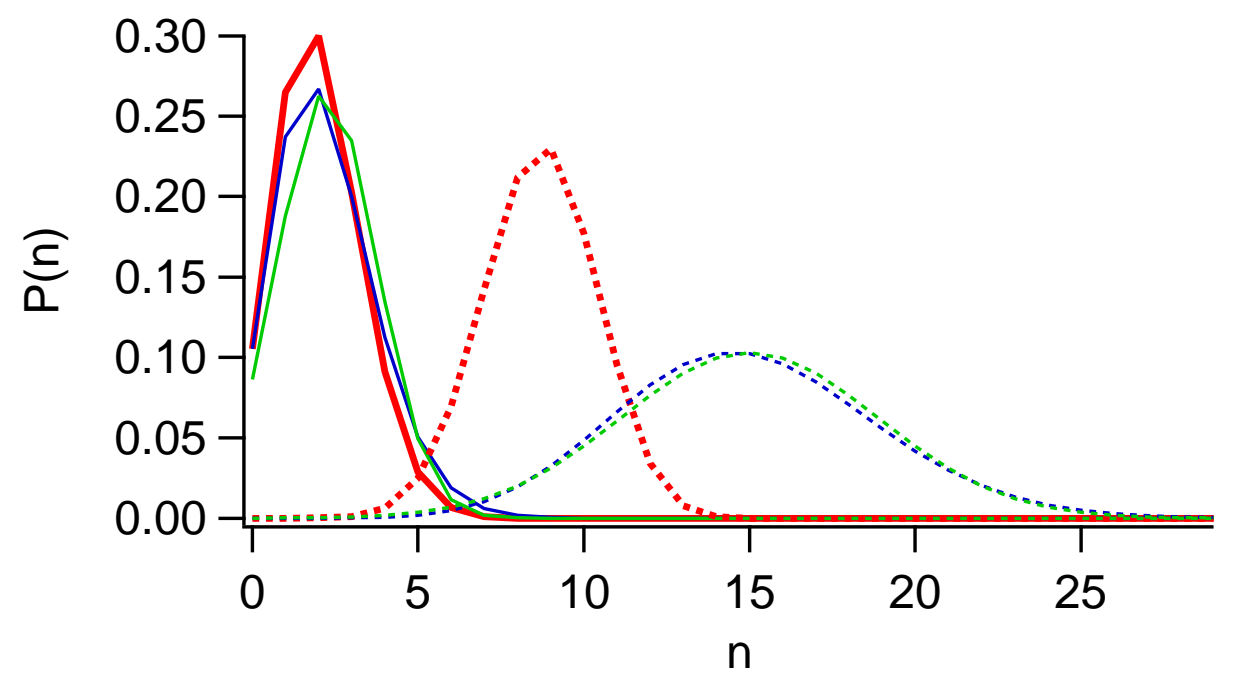

Figure D.2: The solid red line shows the dead-time pdf with $\mu=2.25$. The green and blue solid lines show the Gaussian and Poisson pdf's respectively. The dashed lines show the same pdf's but with $\mu=15$. The Gaussian PDF can almost not be distinguished anymore from the Poisson pdf. 


\section{E Fit with four parameters for non-ideal photon counting processes}

The simulations in section 3.2.5 did not assume any background noise to be present. To test if the effect - that the inverse variance at high photon fluxes is better than expected for dead-time affected counting processes - was a general effect, the following simulation was performed. All simulated 1D Gaussian PSFs had a constant offset of $2\left(A_{\text {off } \text { set }}=2 \frac{\text { photons }}{\mu s}\right.$ in 3.6 and see figure E.1. The data was then simulated for the ideal detection case and fitted with the Poisson-ML estimator (allowing all four parameters in equation 3.6 to be fitted). This result was compared to a simulation where dead-time affected photon counting was present and fitted with the dead-time ML-estimator. Figure E.1 plots the decrease in the inverse variance between these two cases versus the loss of signal photons (integral of the Gaussian PSF after background substraction) caused by the dead-time counting process relative to the ideal counter. One can see, that e.g. when $50 \%$ signal photons are lost due to the dead-time affected counting process, the loss in precision is only $35 \%$ relative to the ideal counting process. In the case of background noise being present, this ratio is signal to noise depended. 

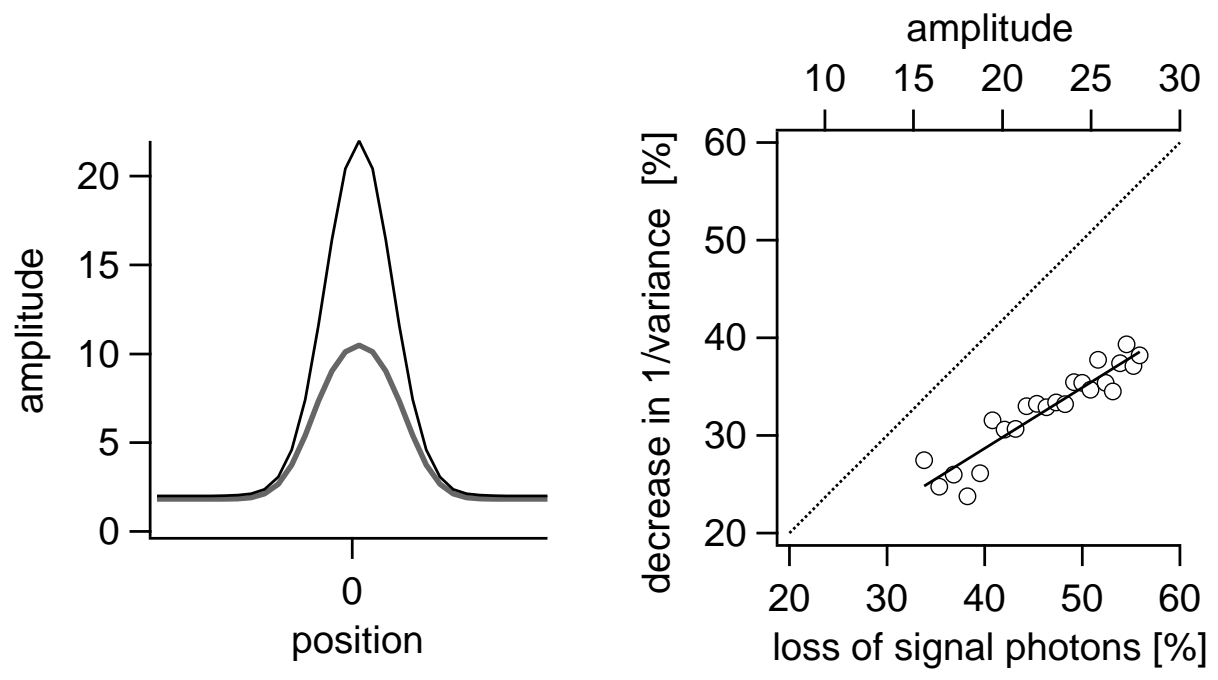

Figure E.1:

Impact of dead-time on the inverse variance in the presence of high background noise. The black line in the left panel shows the PSF of the simulation with $\sigma=2.47$ pixel, $A_{\text {offset }}=2 \frac{\text { photons }}{\mu s}$ and amplitude $A=20 \frac{\text { photons }}{\mu s}$. The grey solid line shows the average of this PSF when measured by a dead-time effected counting process. The right panel plots the relative precision loss vs the photon loss between a simulation with Poisson noise and fit with the Poisson ML-estimator (ideal counting process), and simulation of dead-time affected counting fitted with the dead-time ML-estimator (dead-time affected counting process). The top axis gives the amplitude of the $P S F_{\text {ideal }}^{\text {theory }}$. Points for simulations with very low amplitudes of the PSF are excluded from this plot, since the fits at a too low signal to background ratio had to many outliers. The black line shows a line fit with slope 0.62 and intercept 3.8. Please note, that the variability in this plot comes from outliers. This happened, when the fit did not converge properly. Outliers that were magnitudes off from the averages were removed prior to data analysis. A diagonal dashed black line is plotted for better orientation. 


\section{Bibliography}

Akaike, H. (1981). Likelihood of a model and information criteria. Jornal of Econometrics, $16(1): 3-14$

Albillos, A., Dernick, G., Horstmann, H., Almers, W., Alvarez de Toledo, G., and Lindau, M. (1997). The exocytotic event in chromaffin cells revealed by patch amperometry. Nature, 389(6650):509-12.

Aravanis, A. M., Pyle, J. L., Harata, N. C., and Tsien, R. W. (2003a). Imaging single synaptic vesicles undergoing repeated fusion events: kissing, running, and kissing again. Neuropharmacology, 45(6):797-813.

Aravanis, A. M., Pyle, J. L., and Tsien, R. W. (2003b). Single synaptic vesicles fusing transiently and successively without loss of identity. Nature, 423(6940):643-7.

Axelrod, D., Thompson, N. L., and Burghardt, T. P. (1983). Total internal inflection fluorescent microscopy. J Microsc, 129 Pt 1:19-28.

Berland, K. M., So, P. T., and Gratton, E. (1995). Two-photon fluorescence correlation spectroscopy: method and application to the intracellular environment. Biophys J, 68(2):694701.

Betz, W. J. and Henkel, A. W. (1994). Okadaic acid disrupts clusters of synaptic vesicles in frog motor nerve terminals. J Cell Biol, 124(5):843-54.

Betz, W. J., Mao, F., and Bewick, G. S. (1992). Activity-dependent fluorescent staining and destaining of living vertebrate motor nerve terminals. $J$ Neurosci, 12(2):363-75.

Brakenhoff, G. (1979). Imaging modes in confocal scanning light microscopy (cslm). Jorn. Micro, 117(2):233-242.

Brakenhoff, G., Blom, P., and P., B. (1979). confocal scanning light microscope with high aperture immersion lenses. Jorn. Micro, 117(2):219-232.

Brown, E. B., Shear, J. B., Adams, S. R., Tsien, R. Y., and Webb, W. W. (1999). Photolysis of caged calcium in femtoliter volumes using two-photon excitation. Biophys $J, 76(1 \mathrm{Pt}$ 1):489-99.

Burke, N. V., Han, W., Li, D., Takimoto, K., Watkins, S. C., and Levitan, E. S. (1997). Neuronal peptide release is limited by secretory granule mobility. Neuron, 19(5):1095-102.

Ceccarelli, B., Hurlbut, W. P., and Mauro, A. (1973). Turnover of transmitter and synaptic vesicles at the frog neuromuscular junction. J Cell Biol, 57(2):499-524.

Cheezum, M. K., Walker, W. F., and Guilford, W. H. (2001). Quantitative comparison of algorithms for tracking single fluorescent particles. Biophys J, 81(4):2378-88. 
Corle, T. and Kino, G. (1996). Confocal Scanning Optical Microscopy and Related Imaging Systems. Academic Press.

Cramer, H. (1958). Mathematical Methods of Statistics. Princeton, Univ. Press, New Jersey.

Crank, J. (1975). The Mathematics of Diffusion. Calrendon, London.

De Camilli, P., Cameron, R., and Greengard, P. (1983). Synapsin i (protein i), a nerve terminal-specific phosphoprotein. i. its general distribution in synapses of the central and peripheral nervous system demonstrated by immunofluorescence in frozen and plastic sections. J Cell Biol, 96(5):1337-54.

Denk, W., Strickler, J. H., and Webb, W. W. (1990). Two-photon laser scanning fluorescence microscopy. Science, 248(4951):73-6.

Dunaevsky, A. and Connor, E. A. (2000). F-actin is concentrated in nonrelease domains at frog neuromuscular junctions. J Neurosci, 20(16):6007-12.

Evans, L. L., Lee, A. J., Bridgman, P. C., and Mooseker, M. S. (1998). Vesicle-associated brain myosin-v can be activated to catalyze actin-based transport. J Cell Sci, 111 ( Pt 14):2055-66.

Fessler, J. A. and Yu, D. F. (2000). Mean and variance of single photon counting with deadtime. Phs. Med. Biol., 45:2043-2056.

Fischer, M., Kaech, S., Knutti, D., and Matus, A. (1998). Rapid actin-based plasticity in dendritic spines. Neuron, 20(5):847-54.

Fotin, A., Cheng, Y., Grigorieff, N., Walz, T., Harrison, S. C., and Kirchhausen, T. (2004a). Structure of an auxilin-bound clathrin coat and its implications for the mechanism of uncoating. Nature, 432(7017):649-53.

Fotin, A., Cheng, Y., Sliz, P., Grigorieff, N., Harrison, S. C., Kirchhausen, T., and Walz, T. (2004b). Molecular model for a complete clathrin lattice from electron cryomicroscopy. Nature, 432(7017):573-9.

Gandhi, S. P. and Stevens, C. F. (2003). Three modes of synaptic vesicular recycling revealed by single-vesicle imaging. Nature, 423(6940):607-13.

Gennerich, A. and Schild, D. (2000). Fluorescence correlation spectroscopy in small cytosolic compartments depends critically on the diffusion model used. Biophys J, 79(6):3294-306.

Göppert-Mayer, M. (1931). Über elementarakte mit zwei quantensprügen. Ann Phys, 9:27395.

Greengard, P., Valtorta, F., Czernik, A. J., and Benfenati, F. (1993). Synaptic vesicle phosphoproteins and regulation of synaptic function. Science, 259(5096):780-5.

Harata, N., Pyle, J. L., Aravanis, A. M., Mozhayeva, M., Kavalali, E. T., and Tsien, R. W. (2001). Limited numbers of recycling vesicles in small cns nerve terminals: implications for neural signaling and vesicular cycling. Trends Neurosci, 24(11):637-43.

Heintzmann, R. (1999). Resolution Enhancement of Biological Light Microscopic Data. Phd, Rupertus Carola University.

Heintzmann, R. (in press, 2005). Band-limit and appropriate sampling in microscopy. In Celis, editor, Handbook oc Cell Biology. Elsevier. 
Henkel, A. W., Simpson, L. L., Ridge, R. M., and Betz, W. J. (1996). Synaptic vesicle movements monitored by fluorescence recovery after photobleaching in nerve terminals stained with fm1-43. J Neurosci, 16(12):3960-7.

Heuser, J. E. and Reese, T. S. (1973). Evidence for recycling of synaptic vesicle membrane during transmitter release at the frog neuromuscular junction. J Cell Biol, 57(2):315-44.

Hillesheim, L. N. and Muller, J. D. (2003). The photon counting histogram in fluorescence fluctuation spectroscopy with non-ideal photodetectors. Biophys J, 85(3):1948-58.

Hirokawa, N., Sobue, K., Kanda, K., Harada, A., and Yorifuji, H. (1989). The cytoskeletal architecture of the presynaptic terminal and molecular structure of synapsin 1. J Cell Biol, 108(1):111-26.

Holt, M., Cooke, A., Neef, A., and Lagnado, L. (2004). High mobility of vesicles supports continuous exocytosis at a ribbon synapse. Curr Biol, 14(3):173-83.

Holt, M., Cooke, A., Wu, M. M., and Lagnado, L. (2003). Bulk membrane retrieval in the synaptic terminal of retinal bipolar cells. J Neurosci, 23(4):1329-39.

Hopt, A. and Neher, E. (2001). Highly nonlinear photodamage in two-photon fluorescence microscopy. Biophys J, 80(4):2029-36.

Jordan, R. (2000). Anwendung der Fluoreszenz-Korrelations-Spektroskopie zur Untersuchung dynamischer Prozesse in lebenden Zellen. Phd, Georg-August-Universitaet zu Goettingen.

Kaiser, W. and Garrett, C. (1961). Two-photon excitation in caf $f_{2}$ : eur- ${ }^{2-}$ Phys Rev Lett, $7: 229-31$.

Kask, P., Guenther, R., and Axhausen, P. (1997). Statistical accuracy in fluorescence fluctuation experiments. Eur. Biophys J., 25:163-169.

Katz, B. and Miledi, R. (1967). The timing of calcium action during neuromuscular transmission. J Physiol, 189(3):535-44.

Katz, B. and Miledi, R. (1969). Spontaneous and evoked activity of motor nerve endings in calcium ringer. J Physiol, 203(3):689-706.

Klingauf, J., Kavalali, E. T., and Tsien, R. W. (1998). Kinetics and regulation of fast endocytosis at hippocampal synapses. Nature, 394(6693):581-5.

Kraszewski, K., Daniell, L., Mundigl, O., and De Camilli, P. (1996). Mobility of synaptic vesicles in nerve endings monitored by recovery from photobleaching of synaptic vesicleassociated fluorescence. J Neurosci, 16(19):5905-13.

Kraszewski, K., Mundigl, O., Daniell, L., Verderio, C., Matteoli, M., and De Camilli, P. (1995). Synaptic vesicle dynamics in living cultured hippocampal neurons visualized with cy3-conjugated antibodies directed against the lumenal domain of synaptotagmin. J Neurosci, 15(6):4328-42.

Kues, T., Dickmanns, A., Luhrmann, R., Peters, R., and Kubitscheck, U. (2001). High intranuclear mobility and dynamic clustering of the splicing factor u1 snrnp observed by single particle tracking. Proc Natl Acad Sci U S A, 98(21):12021-6. 
Lang, T., Wacker, I., Wunderlich, I., Rohrbach, A., Giese, G., Soldati, T., and Almers, W. (2000). Role of actin cortex in the subplasmalemmal transport of secretory granules in pc-12 cells. Biophys J, 78(6):2863-77.

Magde, D., Elson, E. L., and Webb, W. W. (1972). Thermodynamic fluctuations in a reacting system-measurement by fluorescence correlation spectroscopy. Phys RevLett, $29: 29-61$.

Magde, D., Elson, E. L., and Webb, W. W. (1974). Fluorescence correlation spectroscopy. ii. an experimental realization. Biopolymers, 13(1):29-61.

Malgaroli, A. and Tsien, R. W. (1992). Glutamate-induced long-term potentiation of the frequency of miniature synaptic currents in cultured hippocampal neurons. Nature, $357(6374): 134-9$.

Marsh, M. and McMahon, H. T. (1999). The structural era of endocytosis. Science, 285(5425):215-20.

Mozhayeva, M. G., Sara, Y., Liu, X., and Kavalali, E. T. (2002). Development of vesicle pools during maturation of hippocampal synapses. J Neurosci, 22(3):654-65.

Mueller, V. J. (2004). Imaging of moleuclar events during endocytosis in hippocmapal synapses. PhD thesis, University of Goettigen.

Mueller, V. J., Wienisch, M., Nehring, R. B., and Klingauf, J. (2004). Monitoring clathrinmediated endocytosis during synaptic activity. J Neurosci, 24(8):2004-12.

Murthy, V. N. and Stevens, C. F. (1998). Synaptic vesicles retain their identity through the endocytic cycle. Nature, 392(6675):497-501.

Murthy, V. N. and Stevens, C. F. (1999). Reversal of synaptic vesicle docking at central synapses. Nat Neurosci, 2(6):503-7.

Neves, G. and Lagnado, L. (1999). The kinetics of exocytosis and endocytosis in the synaptic terminal of goldfish retinal bipolar cells. J Physiol, 515 ( Pt 1):181-202.

Pawley, J., editor (1989). Handbook of Biological Confocal Microscopy. Plenum Press.

Pyle, J. L., Kavalali, E. T., Piedras-Renteria, E. S., and Tsien, R. W. (2000). Rapid reuse of readily releasable pool vesicles at hippocampal synapses. Neuron, 28(1):221-31.

Qian, H. and Elson, E. L. (1990a). Distribution of molecular aggregation by analysis of fluctuation moments. Proc Natl Acad Sci U S A, 87(14):5479-83.

Qian, H. and Elson, E. L. (1990b). On the analysis of high order moments of fluorescence fluctuations. Biophys $J, 57(2): 375-80$.

Rea, R., Li, J., Dharia, A., Levitan, E. S., Sterling, P., and Kramer, R. H. (2004). Streamlined synaptic vesicle cycle in cone photoreceptor terminals. Neuron, 41(5):755-66.

Richards, D. A., Guatimosim, C., and Betz, W. J. (2000). Two endocytic recycling routes selectively fill two vesicle pools in frog motor nerve terminals. Neuron, 27(3):551-9.

Rizzoli, S. O. and Betz, W. J. (2004). The structural organization of the readily releasable pool of synaptic vesicles. Science, 303(5666):2037-9.

Rizzoli, S. O. and Betz, W. J. (2005). Synaptic vesicle pools. Nat Rev Neurosci, 6(1):57-69. 
Royle, S. J. and Lagnado, L. (2003). Endocytosis at the synaptic terminal. J Physiol, 553(Pt 2):345-55.

Ryan, T. A. (1999). Inhibitors of myosin light chain kinase block synaptic vesicle pool mobilization during action potential firing. J Neurosci, 19(4):1317-23.

Ryan, T. A., Li, L., Chin, L. S., Greengard, P., and Smith, S. J. (1996a). Synaptic vesicle recycling in synapsin i knock-out mice. J Cell Biol, 134(5):1219-27.

Ryan, T. A., Reuter, H., and Smith, S. J. (1997). Optical detection of a quantal presynaptic membrane turnover. Nature, 388(6641):478-82.

Ryan, T. A., Reuter, H., Wendland, B., Schweizer, F. E., Tsien, R. W., and Smith, S. J. (1993). The kinetics of synaptic vesicle recycling measured at single presynaptic boutons. Neuron, 11(4):713-24.

Ryan, T. A., Smith, S. J., and Reuter, H. (1996b). The timing of synaptic vesicle endocytosis. Proc Natl Acad Sci U S A, 93(11):5567-71.

Saitoh, M., Ishikawa, T., Matsushima, S., Naka, M., and Hidaka, H. (1987). Selective inhibition of catalytic activity of smooth muscle myosin light chain kinase. J Biol Chem, 262(16):7796-801.

Sandison, D., Williams, R., Wells, K., Strickler, J., and Webb, W. W. (1995). Quantitative fluorescence confocal laser scanning spectrsocopy (clsm). In Pawley, J., editor, Handbook of confocal microscopy. Plenum Press, New York, 2nd edition.

Sankaranarayanan, S., Atluri, P. P., and Ryan, T. A. (2003). Actin has a molecular scaffolding, not propulsive, role in presynaptic function. Nat Neurosci, 6(2):127-35.

Sara, Y., Virmani, T., Deak, F., Liu, X., and Kavalali, E. T. (2005). An isolated pool of vesicles recycles at rest and drives spontaneous neurotransmission. Neuron, 45(4):563-73.

Saxton, M. J. (1993). Lateral diffusion in an archipelago. single-particle diffusion. Biophys $J, 64(6): 1766-80$.

Schikorski, T. and Stevens, C. F. (1997). Quantitative ultrastructural analysis of hippocampal excitatory synapses. J Neurosci, 17(15):5858-67.

Schikorski, T. and Stevens, C. F. (2001). Morphological correlates of functionally defined synaptic vesicle populations. Nat Neurosci, 4(4):391-5.

Sheppard, C., Gan, X., Gu, M., and Roy, M. (1995). Signal-to-noise in confocal microscopes. In Pawley, J., editor, Handbook of confocal microscopy. Plenum Press, New York, 2nd edition.

Shupliakov, O., Bloom, O., Gustafsson, J. S., Kjaerulff, O., Low, P., Tomilin, N., Pieribone, V. A., Greengard, P., and Brodin, L. (2002). Impaired recycling of synaptic vesicles after acute perturbation of the presynaptic actin cytoskeleton. Proc Natl Acad Sci USA, 99(22):14476-81.

Staal, R. G., Mosharov, E. V., and Sulzer, D. (2004). Dopamine neurons release transmitter via a flickering fusion pore. Nat Neurosci, 7(4):341-6.

Steyer, J. A. and Almers, W. (1999). Tracking single secretory granules in live chromaffin cells by evanescent-field fluorescence microscopy. Biophys J, 76(4):2262-71. 
Steyer, J. A., Horstmann, H., and Almers, W. (1997). Transport, docking and exocytosis of single secretory granules in live chromaffin cells. Nature, 388(6641):474-8.

Sudhof, T. C. (2000). The synaptic vesicle cycle revisited. Neuron, 28(2):317-20.

Takei, K., Mundigl, O., Daniell, L., and De Camilli, P. (1996). The synaptic vesicle cycle: a single vesicle budding step involving clathrin and dynamin. J Cell Biol, 133(6):1237-50.

Tan, Y. P., Llano, I., Hopt, A., Wurriehausen, F., and Neher, E. (1999). Fast scanning and efficient photodetection in a simple two-photon microscope. J Neurosci Methods, 92(1-2):123-35.

Thompson, N. (1989). Fluorescence correlation spectroscopy. In Lakowicz, J., editor, Topics in Fluorescence Spectroscopy, volume 1. Plenum Press, New York.

Thompson, R. E., Larson, D. R., and Webb, W. W. (2002). Precise nanometer localization analysis for individual fluorescent probes. Biophys $J, 82(5): 2775-83$.

Verstreken, P., Kjaerulff, O., Lloyd, T. E., Atkinson, R., Zhou, Y., Meinertzhagen, I. A., and Bellen, H. J. (2002). Endophilin mutations block clathrin-mediated endocytosis but not neurotransmitter release. Cell, 109(1):101-12.

Young, J. Z. and Roberts, F. (1951). A flying-spot microscope. Nature, 167(4241):231.

Zenisek, D., Steyer, J. A., and Almers, W. (2000). Transport, capture and exocytosis of single synaptic vesicles at active zones. Nature, 406(6798):849-54. 


\section{Acknowledgement}

First of all, I want to thank Professor Dr. Neher for his commitment to my work during my stay in his department and for advising me on my thesis. I am also grateful to Professor Dr. Troe for serving as the referee of this thesis.

I want to acknowledge Dr. Jürgen Klingauf, who gave me the opportunity to work on the fascinating projects on vesicle dynamics and for his support. I am deeply indebted to Dr. Rainer Heintzmann for the time he spent answering all my questions, and for the various scientific discussions regarding particle tracking techniques.

I want to thank the axes of wisdom in the lab, Martin Wienisch and Alexey Kochubey. Martin was a wonderful colleague, and a constant source of moral support and valuable information on neurobiology. Alexey Kochubey is a multitalent and was always helpful and very patient regarding any mathematical, physical, technical and computational problems I had. I want to thank Dr. Dinah Loerke for being the second source of moral support, and for her fortitude in proofreading my thesis. For reading this thesis I also owe many thanks to Dr. Ina Frambach, Dr. Veronika Müller and Dr. Christian Rochford.

I also want to acknowledge all the other people in the department, especially those who worked with me at the outermost edge of the institute, these are Anna Botvinnik, Dr. Pieter Vanden Berghe, Amitava Mazumdar, Dr. Maria Krikunova and a few meters further, Isabella Gekel, for making my stay in Göttingen very pleasant.

I want to thank Dr. Ralf Nehring for teaching me molecular biology, Michael Pilot for taking care of the neuronal cell culture, Irmgard Barteczko, Frank Würrihausen, as well as Frank Köhne and Ina Herfort for their assistance in various things.

I acknowledge Dr. Randolf Jordan for the good collaboration via e-mail and telephone.

I am obliged to the people in the precision mechanics workshop for the excellent custom-made parts that made my research possible.

Last but not least, I want to express my gratitude to the Boehringer Ingelheim Fond, especially the persons behind it -Dr. Hermann Fröhlich, Monika Beutelspacher and Dr. Claudia Walther- not just for the generous support, but also for the very nice time during the numerous meetings and seminars. Additionally, I would like to thank the graduate college 723 and Professor Dr. Dr. Schild for their support. 



\title{
Curriculum Vitae
}

\author{
Persönliche Angaben
}

Name: Edward A. Lemke

Geboren: 26.01.1978 in Berlin

Staatsangehörigkeit: Deutsch

Aug. 2004

Summerschool am Marine Biological Laboratory in Woods Hole, USA

Feb. 2003 - März 2005 Promotionsstipendium des Boehringer Ingelheim Fonds

Juni 2002

Erwin-Stephan-Preis der Technischen Universität Berlin

Mai 2002 - Jan. 2003

Promotionsstipendium des Graduiertenkollegs 723

seit Feb. 2002

Dez. 2001

Mai 2001

Feb. 2001

Wissenschaftlicher Mitarbeiter am Max-Planck-Institut für biophysikalische Chemie, Abteilung Membranbiophysik (Direktor: Prof. Dr. Erwin Neher)

Jan. 2001 - Feb. 2002

Diplom in Chemie, Technische Universität Berlin, Titel der Diplomarbeit: Biophysical Characterization of FepA Mutants. Referenten: Prof. Dr. Dick van der Helm (University of Oklahoma, USA), Prof. Dr. Wolfgang Lubitz (Technische Universität Berlin)

Master of Science, University of Oklahoma, USA

Aufnahme in die Studienstiftung des Deutschen Volkes

Studentische Hilfskraft mit Lehraufgaben am StranskiLaboratorium für physikalische Chemie der Technischen Universität Berlin

Aug. 1999 - Sep. 2000 Studium an der University of Oklahoma, USA, Department of Chemistry and Biochemistry, Austauschstudent

Aug. 1999

Vordiplom in Chemie, Technische Universität Berlin

Dez. 1998

Okt. 1997 - Dez. 2001 Klaus-Koch-Stipendium der Technischen Universität Berlin Studium der Chemie an der Technischen Universität Berlin, Fakultät für Chemie

Aug. 1997 - Okt. 1997

Galvanikhelfer bei der Firma Siemens

Aug. 1996 - Sep. 1997

Juli 1996

Zivildienst in der Sozialstation in Falkensee

Abitur am Kant-Gymnasium in Berlin 